\title{
Termination Kinetics of Radical Homo- and Copolymerizations Studied via Time- Resolved EPR Spectroscopy
}

\author{
Dissertation \\ for the award of the degree \\ "Doctor rerum naturalium" (Dr.rer.nat.) \\ of the Georg-August-Universität Göttingen \\ within the doctoral program chemistry \\ of the Georg-August University School of Science (GAUSS) \\ submitted by
Lara Riemann
from Witzenhausen
}

Göttingen, 2021 
Thesis Committee:

Prof. Dr. Philipp Vana, MBA Institute of Physical Chemistry Georg-August University Göttingen

Prof. Dr. Thomas Zeuch Institute of Physical Chemistry Georg-August University Göttingen

Members of the Examination Board:

Reviewer:

Prof. Dr. Philipp Vana, MBA Institute of Physical Chemistry Georg-August University Göttingen

Second Reviewer:

Prof. Dr. Thomas Zeuch

Institute of Physical Chemistry

Georg-August University Göttingen

Further members of the Examination Board:

Prof. Dr. Michael Buback Institute of Physical Chemistry

Georg-August University Göttingen

Prof. Dr. Burkhard Geil

Institute of Physical Chemistry

Georg-August University Göttingen

Prof. Dr. Dietmar Stalke

Institute of Inorganic Chemistry

Georg-August University Göttingen

Dr. Tim Schäfer

Institute of Physical Chemistry

Georg-August University Göttingen

Date of oral examination:

14.04.2021 
This thesis provides an in-depth investigation into the chain-length dependent termination kinetics of radical homo- and copolymerization by using the most powerful method: single pulse-pulsed laser polymerization (SP-PLP) in conjunction with electron paramagnetic resonance (EPR) spectroscopy. In combination with the kinetic simulation package PREDICI ${ }^{\circledR}$, more detailed insights into the complex copolymerization kinetics were obtained.

For the first time, the composite parameters for the radical homopolymerization of $n$-pentyl methacrylate (PnMA) in bulk were determined over a wide temperature range. The composite parameters $\alpha_{\text {s }}$ and $\alpha_{1}$, which describe the strength of the chain-length dependence of the termination, perfectly agrees with both literature values for other methacrylates and the theoretically predicted values. Furthermore, the activation energy $E_{\mathrm{A}}\left(k_{\mathrm{t}}^{1,1}\right)$ for the termination rate coefficient of two monomeric radicals was obtained by an Arrhenius plot. Here, $k_{\mathrm{t}}^{1,1}$ showed a clear relationship with the viscosity $\eta$ and it was observed that the product $k_{\mathrm{t}}^{1,1} \cdot \eta$ is insensitive toward temperature and that the value of $k_{\mathrm{t}}^{1,1} \cdot \eta$ depends on the hydrodynamic radius. All the results of the PnMA polymerization fitted perfectly into the trends within the methacrylate family.

Furthermore, the homopolymerization of 2-ethylhexyl methacrylate (2-EHMA) and dodecyl methacrylate (DMA) was extensively studied in this thesis with the focus on the temperature dependency of the crossover chain length $i_{\mathrm{c}}$. A sigmoidal behavior of the crossover chain length could be observed for both monomers where $i_{\mathrm{c}}$ decreases with increasing temperature. Such a temperature dependency of $i_{c}$ could not be observed for PnMA. Hence, it could be demonstrated that the size of ester side chain significantly influences the crossover chain length. This was also confirmed by the determined inflection points of the sigmoidal fits of the 
experimental data for 2-EHMA and DMA. The inflection point of 2EHMA was at a significantly lower temperature than for DMA. On this point, more experimental data on the chain-length dependent termination kinetics for several methacrylates (PnMA, 2-EHMA and DMA) were obtained within this work. Furthermore, parts of the experimental setup had to be replaced. Thus, these changes could be validated by the investigated methacrylates in this work because they fit perfectly into the tendencies of the other methacrylates.

In the second part of this thesis, the kinetics of the radical copolymerization of styrene and MMA was thoroughly investigated. First, EPR spectra were measured for different compositions. It is worthmentioning that fully deuterated styrene-d8 was used to simplify the EPR spectra. By comparing the corresponding homopolymerization spectra to the spectrum obtained for the copolymerization, a clear assignment of the different signals in the copolymerization spectra to the macroradicals with the different terminal units was achieved. Moreover, the radical fraction of styrene was directly determined by fitting the EPR spectra via Matlab $^{\circledR}$. The so-obtained radical fraction of styrene-d8 was significantly higher than the feed fraction of styrene-d8. For understanding this behavior, the penultimate model was applied in conjunction the literature known copolymerization parameters. However, this approach failed to describe the radical fraction of styrene. If the copolymerization parameters were adjusted to the radical fraction of styrene, the model were not able to describe the literature known propagation rate coefficient of the copolymerization and copolymer composition. To address this issue, it was manually analyzed whether a set of copolymerization parameters can describe all experimental data simultaneously. This approach was successful for both penultimate models. In this way, more reliable copolymerization parameters were received. Furthermore, single pulse experiments were reevaluated with a refined PREDICI ${ }^{\circledR}$ model. To do so, four simulation approaches were applied considering the following four aspects: (A) viscosity of the copolymerization mixture, (B) termination reactions, (C) copolymerization parameters and (D) chain lengths of macroradicals. Depending on the simulation variant, $k_{\mathrm{t}, \text { cross }}^{1,1}$ and $k_{\mathrm{t}, \text { copo }}^{1,1}$ was determined via PREDICI ${ }^{\circledR}$ modelling based on the implicit penultimate model with a 
parameter estimation. If the termination reactions were separated into cross-termination and homo-terminations under consideration of the viscosity of the copolymerization (simulation variant $\mathrm{A}$ ), no realistic values were obtained for cross-termination rate coefficient $k_{t, \text { cross }}^{1,1}$. Therefore, the termination reactions were treated equally in another simulation (simulation variant B). Thus, a more realistic $k_{\mathrm{t}, \mathrm{copo}}^{1,1}$ was received. Since the copolymerization parameters from literature could not describe the radical fraction of styrene, a further simulation (simulation variant C) was performed with the manually fitted copolymerization parameters, and hence a realistic $k_{\mathrm{t}, \text { copo }}^{1,1}$ was successfully obtained. In the last simulation (simulation variant D), different chain length of the macroradicals were considered. The soobtained $k_{t, \text { copo }}^{1,1}$ was equal to the diffusion limit which is considered to be unrealistic. Nonetheless, $k_{\mathrm{t}, \mathrm{copo}}^{1,1}$ was significantly higher than $k_{\mathrm{t}}^{1,1}$ for the corresponding homopolymerizations in all simulation variants which agrees with results from previous works. This might be explained by a different chain flexibility of the copolymeric macroradicals compared to the homopolymeric case. Combing all the simulation results with the experimentally determined parameters, the whole kinetic picture of copolymerization system of MMA and styrene finally becomes clearer and more comprehensive. This combined method of SP-PLP-EPR and PREDICI ${ }^{\circledR}$ simulation opens up new perspectives for both experimental and theoretical approaches for the in-depth investigation into the kinetics of radical copolymerizations. 



\section{CONTENT}

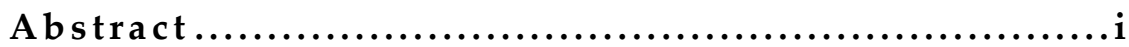

1 Introduction and Motivation ........................ 1

2 Theoretical Background ........................... 5

2.1 Ideal Kinetics of Radical Homopolymerizations.............................. 5

2.1.1 Initiation of Radical Homopolymerizations ........................... 5

2.1.2 Propagation of Radical Homopolymerizations ................... 7

2.1.3 Termination of Radical Homopolymerizations ................... 7

2.1.4 Steady-State Polymerization Rate........................................... 8

2.2 Transfer Reactions of Radical Homopolymerizations...................... 8

2.3 Diffusion Control of Radical Homopolymerizations ..................... 9

2.3.1 Diffusion Control of Initiation................................................ 9

2.3.2 Diffusion Control of Propagation ........................................ 10

2.3.3 Diffusion Control of Termination .......................................... 11

2.4 Chain-Length Dependency of Radical Homopolymerizations.. 13

2.4.1 Chain-Length Dependency of Propagation........................ 13

2.4.2 Chain-Length Dependency of Termination ....................... 14

2.5 Kinetics of Radical Copolymerizations............................................ 16

2.5.1 Initiation of Radical Copolymerizations .............................. 17

2.5.2 Propagation of Radical Copolymerizations ....................... 17

2.5.3 Termination of Radical Copolymerizations ........................ 22

2.6 SP-PLP-EPR Method ...................................................................... 24

3 Experimental ...................................... 27

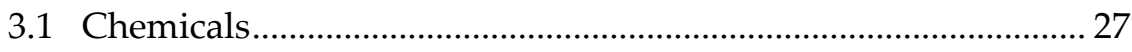




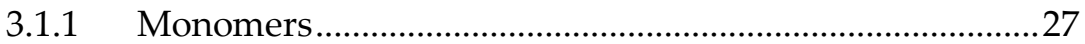

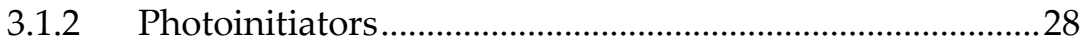

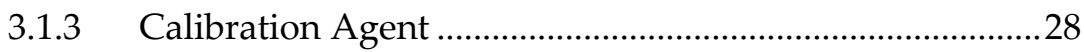

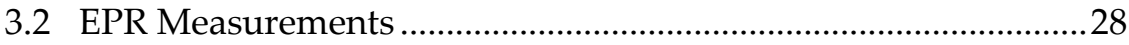

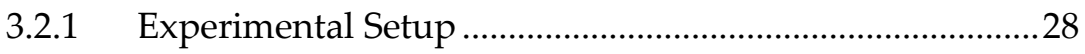

3.2.2 Experimental Procedure .........................................................30

3.2.3 Sample Preparation ..................................................................30

3.2.4 Choice of Photoinitiator...........................................................31

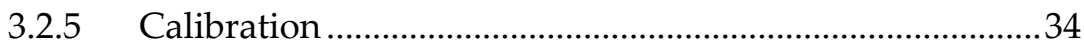

3.2.6 Determination of Conversion ................................................35

3.2.7 Density and Viscosity Measurements...................................36

3.2.8 Kinetic Simulations...................................................................

3.2.9 Simulations of the EPR Spectra …………………………......37

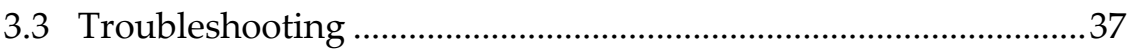

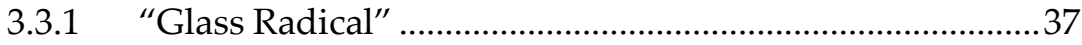

3.3.2 EPR Signal of Oxygen .......................................................... 38

3.3.3 EPR Signal of the Heating Element........................................39

4 Termination Kinetics of Radical Polymerization of Methacrylates ...................................... 41

4.1 EPR Spectrum of Pentyl Methacrylate .............................................42

4.2 Chain-Length Dependent Termination of Pentyl Methacrylate.44

4.3 Crossover Chain Length for Various Methacrylates......................52

4.4 Conclusion and Further Perspectives................................................58

5 Kinetics of Radical Copolymerizations .............61 61

5.1 EPR Spectra of Homopolymerizations and Copolymerizations 62

5.2 Determination of the Styrene Radical Fraction .71 


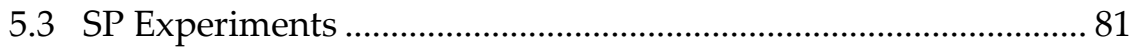

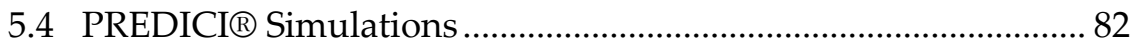

5.4.1 Influence of Different Termination Reactions .................. 84

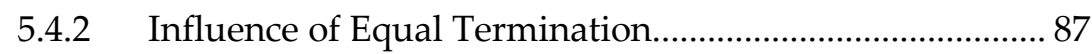

5.4.3 Influence of Copopolymerization Parameters .................. 90

5.4.4 Influence of Different Chain Lengths .............................. 92

5.5 Conclusion and Further Perspectives ..................................... 97

6 Closing Remarks ............................... 101

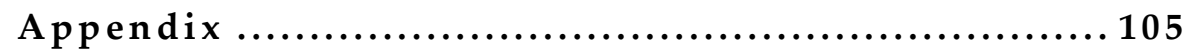

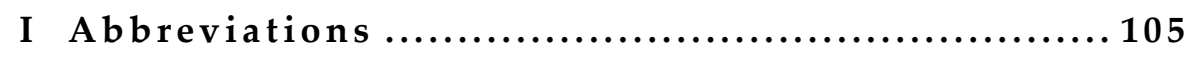

II Figures And Tables ............................... 111

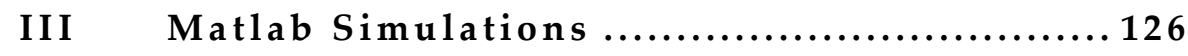

IV Literature.................................. 131 



\section{INTRODUCTION AND MOTIVATION}

The history of synthetic polymers started around 100 years ago. The first synthetic polymer was synthesized by Bakeland ${ }^{[1]}$ and the first description of a polymerization was written by Staudinger. ${ }^{[2]}$ These works opened the triumphant path of polymers, and thus nowadays polymeric products are indispensable because of their versatile applications. Beside the household items made from polymers such as PET bottles or plastic bags, polymers are used in a wide spectrum across the automobile construction, airplanes construction, medical products and nearly

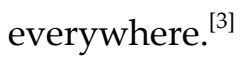

The conventional radical polymerization is the most used polymerization type due to the undemanding conditions in comparison to other polymerizations techniques. ${ }^{[4]}$ The interest into the kinetics of radical polymerizations is immense for the precise control of the polymerization process and the creation of a well-defined polymeric product in laboratory and industry. However, it took decades to obtain precise rate coefficients. ${ }^{[5]}$ The development of different pulsed laser polymerization (PLP) methods was a great step forward to determine precise rate coefficients. ${ }^{[6]}$ Olaj et. al. analyzed the product after PLP with size exclusion chromatography (SEC), and thus the propagation rate coefficient $k_{\mathrm{p}}$ can be determined (PLP-SEC). ${ }^{[7]}$ This method is validated and recommended by IUPAC ${ }^{[8-10]}$ and was successfully used for several homo- and copolymerizations. ${ }^{[11-23]}$ Moreover, further insights into the termination kinetics can be obtained by a single laser pulse (SP) and the online determination of the monomer consumption via near-infrared (NIR) spectroscopy (SP-PLP-NIR) ${ }^{[6,24]}$ However, this method only provides the chain-length-averaged termination rate coefficient due to the limitations of NIR. ${ }^{[25]}$ Furthermore, Buback et al. established the first method which provides a direct insight into the chain-length dependent termination kinetics. ${ }^{[25-27]}$ Therefore, the decay of the radical 
concentration is directly measured after a single laser pulse via timeresolved electron paramagnetic resonance (EPR) spectroscopy (SP-PLP$\mathrm{EPR}) \cdot{ }^{[27,28]}$ With this method, the composite parameters can be determined. ${ }^{[29]}$ This method was used for several homopolymerizations in bulk ${ }^{[26,30-35]}$ and solutions. ${ }^{[2,36-40]}$ Additionally, information about two coexisting radicals can be received. Thus, investigations into backbiting of acrylates and acrylamide are possible because the secondary propagating radicals (SPR) and the mid chain radicals (MCR) can be clearly identified. ${ }^{[36,39,41-43]}$ Consequently, also investigations into controlled polymerizations such as $\mathrm{RAFT}^{[44-46]}$ and ATRP ${ }^{[25]}$ respectively, were performed, too. Moreover, it was possible to determine $k_{\mathrm{p}}$ for ionic monomers via SP-PLP-EPR. ${ }^{[4]}$ All this demonstrate the outstanding advantages of SP-PLP-EPR as a powerful and versatile tool for the study of complex polymerizations kinetics.

Furthermore, the radical copolymerization is an important polymerization technique to create a copolymer which processes the properties of both homopolymers. ${ }^{[4]}$ Therefore, the applications of copolymers are broad and they are used, for instance, as binder resin in automotive coating, rubber compounds, textiles and foils. ${ }^{[3,49]}$ For radical copolymerizations, the knowledge of the kinetics is of great importance, since it influences the composition, sequence distribution, molecular weight distribution and chain-end composition of the copolymer and all this detailed change in the copolymer architecture have an huge impact on the demanded performance of the copolymer. ${ }^{[5]}$ In this way, the kinetic controlling becomes the most straight forward and effective approach for the engineering on diverse properties of the copolymer product. Nonetheless, due to the complex kinetics of copolymerizations even the determination of the propagation of copolymerizations was challenging. ${ }^{[5,18,50-52]}$ Models are required to describe the copolymerization kinetics. ${ }^{[5]}$ First, the terminal model was developed where it is assumed that only the terminal unit of a macroradical influences the propagation kinetics. ${ }^{[5]}$ However, after obtaining reliable propagation rate coefficients of copolymerizations $k_{\mathrm{p} \text {, copo }}$ via PLP-SEC, it was shown that the terminal model fails to describe the experimental $k_{\mathrm{p}, \text { copo }}{ }^{[50]}$ Therefore, other models 
were regarded again. The most promising ones is the penultimate model where the penultimate and terminal units affect the propagation kinetics and which can describe the copolymer composition and $k_{\mathrm{p}, \text { cop. }}{ }^{[50]}$ However, the determination of the copolymerization parameters is still uncertain. ${ }^{[52]}$ Moreover, the chain-length dependent termination kinetics of radical copolymerizations was investigated only one time via a stationary method..$^{[53]}$

First investigations into the chain-length dependent termination kinetics of radical copolymerization are performed within this work using the outstanding SP-PLP-EPR method. Therefore, single pulse experiments of the radical copolymerization of styrene-d8 and MMA in bulk are evaluated with PREDICI ${ }^{\circledR}$. This PREDICI ${ }^{\circledR}$ model bases on the implicit penultimate model and several aspects such as the viscosity, equal termination reactions, copolymerization parameters and different chain lengths of the macroradicals are analyzed. Hence, $k_{\mathrm{t}, \text { copo }}^{1,1}$ is determined via parameter estimation. Additionally, EPR spectra of the styrene-d8-MMA copolymerization for different compositions are measured. Thus, the radical fraction of styrene-d 8 is determined directly from theses EPR spectra with Matlab ${ }^{\circledR}$. With this additional experimental data, more precise copolymerization parameters can be obtained.

Beside the radical copolymerization, the termination kinetics of the radical homopolymerization of methacrylates are investigated via SPPLP-EPR. The propagation and termination kinetics are well known for the most methacrylates. Poly-pentyl methacrylate is an interesting matrix polymer for testing the mechanical properties of polymer-filler compounds because its glass transition temperature is slightly below room temperature ${ }^{[54]}$ However, PnMA was not kinetically investigated before. Therefore, the composite parameters for PnMA are determined. Furthermore, for 2-ethylhexyl methacrylate (2-EHMA) and dodecyl methacrylate (DMA), a temperature dependence of $i_{\mathrm{c}}$ was observed. ${ }^{[55,56]}$ Hence, further investigations into the termination kinetics of these monomers are performed in this thesis to obtain more information about this temperature dependency. Moreover, since important parts of the SP- 
PLP-EPR setup had to be exchanged or repaired, the results of the methacrylates can be compared with literature values, and thus the setup is validated. 


\section{THEORETICAL BACKGROUND}

Conventional radical polymerization (CRP) is one of the most important polymerization types. ${ }^{[4]}$ For planning a radical polymerization and to get a well-defined product-both on laboratory and industrial scale-the knowledge of the kinetics of each polymerization step (initiation, propagation, termination, transfer etc.) is essential. If more than one monomer is polymerized in a radical polymerization, it is called copolymerization and the kinetics of propagation and termination become more complicated due to different reactivities of the monomers (see Chapter 2.5).

\subsection{Ideal Kinetics of Radical Homopolymerizations}

The ideal kinetics of CRP is divided into initiation, propagation and termination and the following assumptions are made: ${ }^{[5]}$

(1) All steps are irreversible.

(2) Monomer is only consumed in the propagation step.

(3) Reactivity of radicals is independent from conversion and chainlength.

(4) Termination of two macroradicals only occurs via combination or disproportionation.

(5) The initiator concentration is constant.

For describing the real radical polymerization kinetics, it is important to include transfer reactions such as backbiting. Moreover, diffusional control and chain-length dependency of each polymerization step must be considered, especially for the termination reaction.

\subsubsection{Initiation of Radical Homopolymerizations}

The initiation of a radical polymerization is divided into two steps. First, the initiator $\mathrm{I}_{2}$ decays and two primary radicals $\mathrm{I}^{\bullet}$ are formed. The decomposition of the initiator is a homolytical bond cleavage which can 
occur photo chemically, thermally or via redox reactions, and is described by the rate coefficient $k_{\mathrm{d}} \cdot{ }^{[5]}$

$$
\mathrm{I}_{2} \stackrel{k_{\mathrm{d}} \cdot f}{\longrightarrow} 2 \mathrm{I}^{\bullet}
$$

However, to initiate the chain growth, the formed primary radicals must leave the solvent cage. Otherwise, due to the so-called "cage-effect", they recombine and cannot initiate the chain growth. This effect is taken into account by the initiator efficiency $f{ }^{[57]}$ The rate of initiator decomposition is described by the following rate law:

$$
\frac{\mathrm{d} c_{\mathrm{I}} \cdot}{\mathrm{d} t}=2 \cdot k_{\mathrm{d}} \cdot f \cdot c_{\mathrm{I}},
$$

where $c_{I}$ is the concentration of primary radicals, $t$ the time and $c_{I}$ the concentration of initiator.

The second step is the initiation of the chain growth where the formed primary radicals add monomers $\mathrm{M}$ and monomeric radicals $\mathrm{P}_{1}^{*}$ are generated.

$$
\mathrm{I}^{\bullet}+\mathrm{M} \stackrel{k_{\mathrm{i}}}{\longrightarrow} \mathrm{P}_{1}^{\bullet}
$$

This initiation step is given by the ensuing rate law:

$$
\frac{\mathrm{d} c_{\mathrm{P} i}}{\mathrm{~d} t}=k_{\mathrm{i}} \cdot c_{\mathrm{M}} \cdot c_{\mathrm{I}} \text {. }
$$

Here, $c_{\mathrm{M}}$ is the monomer concentration, $c_{\mathrm{P}_{1}}$ the monomeric radical concentration and $k_{\mathrm{i}}$ the rate coefficient for the initiation step. However, for the most suitable initiators, this initiation step can be assumed to be faster than the propagation, and thus it does not affect the overall kinetics. Moreover, some monomers are able to undergo a self-initiation. For

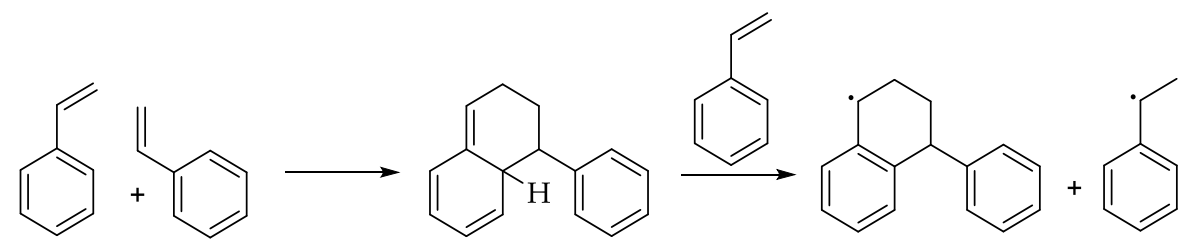

Figure 2.1: Self-initiation of styrene via Diels-Alder cycloaddition. ${ }^{[5]}$ 
instance, in Figure 2.1 the self-initiation via a Diels-Alder cycloaddition of styrene is depicted. ${ }^{[58]}$ As experiments have shown, the self-initiation is only relevant for high temperatures. ${ }^{[5]}$

\subsubsection{Propagation of Radical Homopolymerizations}

During the propagation, a macroradical $\mathrm{P}_{i}^{\bullet}$ with the chain length $i$ is formed by continuously adding monomer units. The propagation is represented by the rate coefficient $k_{\mathrm{p}}{ }^{[5]}$

$$
\mathrm{P}_{i}^{\bullet}+\mathrm{M} \stackrel{k_{\mathrm{p}}}{\longrightarrow} \mathrm{P}_{i+1}^{\bullet}
$$

The propagation rate law is given by:

$$
\frac{\mathrm{d} c_{\mathrm{M}}}{\mathrm{d} t}=-k_{\mathrm{p}} \cdot c_{\mathrm{M}} \cdot c_{\mathrm{R}}
$$

with $c_{\mathrm{R}}$ as macroradical concentration.

\subsubsection{Termination of Radical Homopolymerizations}

Finally, two macroradicals $\mathrm{P}_{i}^{\bullet}$ and $\mathrm{P}_{j}^{\bullet}$ terminate by combination or disproportionation. The combination of two macroradicals results in one saturated macromolecule $\mathrm{P}_{i+j}$. During disproportionation, a hydrogen is transferred from one macroradical to the other, and thus one saturated $\mathrm{P}_{j}^{\mathrm{H}}$ and one unsaturated macromolecule $\mathrm{P}_{i}^{=}$is formed. ${ }^{[5]}$

$$
\begin{gathered}
\mathrm{P}_{i}^{\cdot}+\mathrm{P}_{j}^{\cdot} \stackrel{k_{\mathrm{tk}}}{\longrightarrow} \mathrm{P}_{i+j} \\
\mathrm{P}_{i}^{\cdot}+\mathrm{P}_{j}^{\cdot} \stackrel{k_{\mathrm{td}}}{\longrightarrow} \mathrm{P}_{i}^{=}+\mathrm{P}_{j}^{\mathrm{H}}
\end{gathered}
$$

Here, $k_{\mathrm{tk}}$ is the rate coefficient for combination and $k_{\mathrm{td}}$ is the rate coefficient for disproportionation. The rate law of the termination is obtained by the following equation:

$$
\frac{\mathrm{d} c_{\mathrm{R}}}{\mathrm{d} t}=-2 \cdot\left\langle k_{\mathrm{t}}\right\rangle \cdot c_{\mathrm{R}}^{2}
$$


with $\left\langle k_{\mathrm{t}}\right\rangle$ as average termination rate coefficient. The IUPACrecommended factor of 2 is considered in Equation 2.4 and throughout this thesis. ${ }^{[59,60]}$

\subsubsection{Steady-State Polymerization Rate}

Under stationary conditions, it is assumed that the amount of generated radicals in the initiation step is equal to the terminating radicals. Thus, it follows:

$$
2 \cdot k_{\mathrm{d}} \cdot f \cdot c_{\mathrm{I}}=2 \cdot\left\langle k_{\mathrm{t}}\right\rangle \cdot c_{\mathrm{R}}^{2} .
$$

Combining Equation 2.3 and 2.5 lead to the subsequent equation for the overall polymerization rate $R_{\mathrm{p}}$ :

$$
R_{\mathrm{p}}=-\frac{\mathrm{d} c_{\mathrm{M}}}{\mathrm{d} t}=k_{\mathrm{p}} \cdot c_{\mathrm{M}} \sqrt{\frac{k_{\mathrm{d}} \cdot f \cdot c_{\mathrm{I}}}{\left\langle k_{\mathrm{t}}\right\rangle}} .
$$

From this equation, it is apparent that the reaction order for $k_{\mathrm{p}}$ and $c_{\mathrm{M}}$ is one, whereas it is 0.5 for $k_{\mathrm{d}}$ and $\left\langle k_{\mathrm{t}}\right\rangle$. It should be noted that for the reaction order of $c_{\mathrm{M}}$ also $\omega$ is used which differs from one if transfer reactions occur. ${ }^{[61]}$

\subsection{Transfer Reactions of Radical Homopolymerizations}

The ideal kinetics of radical polymerizations does not consider transfer reactions where the radical functionality is transferred intermolecularly to another molecule $X$. X can be monomer, initiator, solvent, a dead macromolecule, or an added chain-transfer agent (CTA). The rate coefficient for this transfer step is $k_{\mathrm{tr}}$. The chain growth of the macroradical is stopped but the new generated radical $\mathrm{X}^{\bullet}$ can initiate again which is described by the rate coefficient $k_{\mathrm{p}, \mathrm{X}}$. Thus, the radical concentration stays constant during the polymerization. Such a CTA is often used to control the molecular weight.

$$
\begin{aligned}
& \mathrm{P}_{i}^{\bullet}+\mathrm{X} \stackrel{k_{\mathrm{tr}}}{\longrightarrow} \mathrm{k}_{i}+\mathrm{X}^{\bullet} \\
& \mathrm{X}^{\bullet}+\mathrm{M} \stackrel{k_{\mathrm{p}} \mathrm{C}}{\longrightarrow} \mathrm{X}^{-\mathrm{M}^{\bullet}}
\end{aligned}
$$




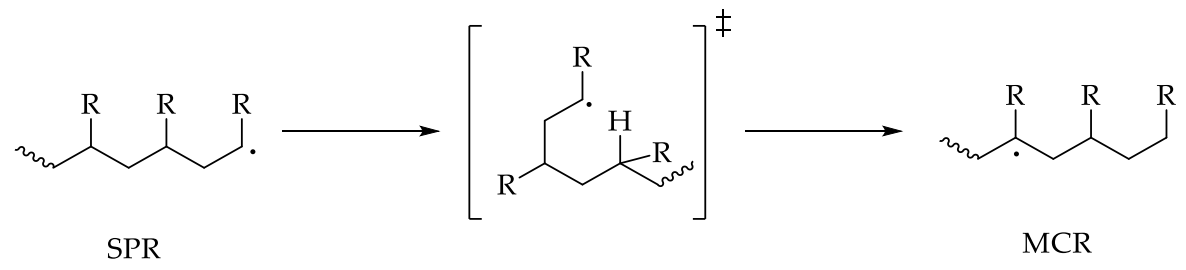

Figure 2.2: Intramolecular transfer of the radical functionality via 1,5-hydrogentransfer (backbiting). During this step, a secondary propagation radical (SPR) is transferred to mid chain radical (MCR) via a six-membered transition state.

Moreover, an important intramolecular transfer of the radical functionality is the 1,5-hydrogen-shift which is known as backbiting (Figure 2.2). ${ }^{[61]}$ The radical functionality of the secondary propagating radical (SPR) is converted via a six-membered transition state to a midchain radical (MCR).

\subsection{Diffusion Control of Radical Homopolymerizations}

For a bimolecular reaction to take place, the reactants must diffuse through the reaction medium. Therefore, it is not surprising that diffusion control must be considered in a radical polymerization. The self-diffusion coefficient $D^{i}$ for radicals with the chain length $i$ is proportional to the fluidity $\eta^{-1}$ and antiproportional to the hydrodynamic ratio $r_{i}$ and is given by the Stokes-Einstein equation (Equation 2.7): ${ }^{[57]}$

$$
D^{i}=\frac{k_{\mathrm{B}} \cdot T}{6 \cdot \pi \cdot r_{i} \cdot \eta}
$$

where $k_{\mathrm{B}}$ is the Boltzmann constant and $T$ the temperature. During a radical polymerization, the viscosity $\eta$ changes by several orders of magnitude, and thus the diffusion process becomes more restricted. Hence, the diffusion-controlled reaction steps of a radical polymerization show significant changes in their kinetics with increasing monomer-topolymer conversion. ${ }^{[57]}$

\subsubsection{Diffusion Control of Initiation}

Although the initiation is not a bimolecular reaction, it must be considered that the primary radicals have to leave the solvent cage to 
initiate the chain growth. Therefore, not the rate coefficient $k_{\mathrm{d}}$ is but rather the initiator efficiency $f$ is diffusion controlled. However, the diffusion control of $f$ is only observable at very high monomer-to-polymer conversion. ${ }^{[57]}$

\subsubsection{Diffusion Control of Propagation}

The diffusion of monomer and macroradicals is relevant for the propagation step. In comparison to macroradicals, monomers can diffuse easily through the reaction medium because of their smaller $r_{i}$ (Equation 2.7). Moreover, the amount of monomer is orders of magnitudes higher compared to the amount of macroradicals, and thus the center-of-mass diffusion of the macroradicals can be neglected. A decrease of $k_{\mathrm{p}}$ is observed merely at conversion above $80 \%$ (glass effect) and the polymerization stops before the full conversion $X$ is reached. ${ }^{[57]}$ Hence, the conversion dependence of $k_{\mathrm{p}}$ is described by Equation 2.8: ${ }^{[62]}$

$$
\frac{1}{k_{\mathrm{p}}}=\frac{1}{k_{\mathrm{p}}^{0}}+\frac{1}{k_{\mathrm{p}, X}}
$$

where $k_{\mathrm{p}}^{0}$ is the propagation rate coefficient at $0 \%$ conversion and $k_{\mathrm{p}, X}$ the propagation rate coefficient which considers the diffusion control (see Equation 2.9). ${ }^{[62]}$

$$
k_{\mathrm{p}, X}=\frac{k_{\mathrm{p}}}{\eta_{\mathrm{r}}(X)}
$$

The change of $\eta$ during the polymerization is expressed by the relative viscosity $\eta_{\mathrm{r}}(X)$ as follows: ${ }^{[62]}$

$$
\eta_{\mathrm{r}}(X)=\frac{\eta}{\eta_{0}}
$$

Here, $\eta_{0}$ is the viscosity at $0 \%$ conversion. Nevertheless, the decrease of $k_{\mathrm{p}}$ is difficult to isolate from these observations because the initiator efficiency is also diffusion-controlled at high conversion. ${ }^{[57]}$ 


\subsubsection{Diffusion Control of Termination}

According to Equation 2.7, macroradicals have a small $D^{i}$, and thus the termination of two macroradicals is diffusion-controlled from the beginning of the polymerization. Benson and North described the diffusion process of two macroradicals and divided it into three steps as shown schematically in Figure 2.4. ${ }^{[63,64]}$ In the first step, the center-of-mass diffusion or so-called translational diffusion (TD) of the two macroradicals occurs, so that a direct contact between them can take place. The two macroradicals start to entangle and their radical chain ends might reach each other by a segmental diffusion (SD). Finally, a chemical reaction (CR) may occur between the two macroradicals which is always the fastest step, and thus it is never rate-determining.

Moreover, Buback developed a model for describing the diffusioncontrolled termination rate coefficient $\left\langle k_{t, d}\right\rangle$ which combines the termination rate coefficients for each diffusion process (Equation 2.11). ${ }^{[62]}$

$$
\left\langle k_{\mathrm{t}, \mathrm{d}}\right\rangle=\frac{1}{\left\langle k_{\mathrm{t}, \mathrm{TD}}\right\rangle}+\frac{1}{\left\langle k_{\mathrm{t}, \mathrm{SD}}\right\rangle}+\frac{1}{\left\langle k_{\mathrm{t}, \mathrm{CR}}\right\rangle}
$$

Investigations of the conversion dependence of $\left\langle k_{\mathrm{t}}\right\rangle$ showed its decrease during the polymerization. This decrease can be divided into different regions relating to the different dominating diffusion steps. For instance, the conversion dependence of $\left\langle k_{\mathrm{t}}\right\rangle$ is shown in Figure 2.3 for methyl methacrylate (MMA) in bulk at $50{ }^{\circ} \mathrm{C}$ taken from literature. ${ }^{[62]}$ In the first region, $\left\langle k_{t}\right\rangle$ stays nearly constant (plateau) and SD is the ratedetermining step. This is followed by a significant decrease of $\left\langle k_{\mathrm{t}}\right\rangle$ because of a viscosity increase and now TD is rate-controlling. Hence, the termination rate coefficient $\left\langle k_{\mathrm{t}, \mathrm{TD}}\right\rangle$, where TD is rate-determining, can be related to $\eta_{\mathrm{r}}$ as follows: ${ }^{[62]}$

$$
\left\langle k_{\mathrm{t}, \mathrm{TD}}\right\rangle=\frac{\left\langle k_{\mathrm{t}, \mathrm{TD}}^{0}\right\rangle}{\eta_{\mathrm{r}}},
$$

where $\left\langle k_{\mathrm{t}, \mathrm{TD}}^{0}\right\rangle$ is the (hypothetical) rate coefficient at $0 \%$ conversion. Furthermore, the radical concentration, the polymerization rate, and the conversion increase significantly and lead to the gel-effect. ${ }^{[57]}$ In the third 

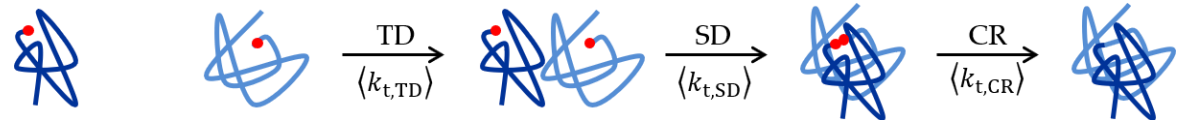

Figure 2.4: Schematic illustration of the diffusion process of two macroradicals divided into three steps introduced by Benson and North.

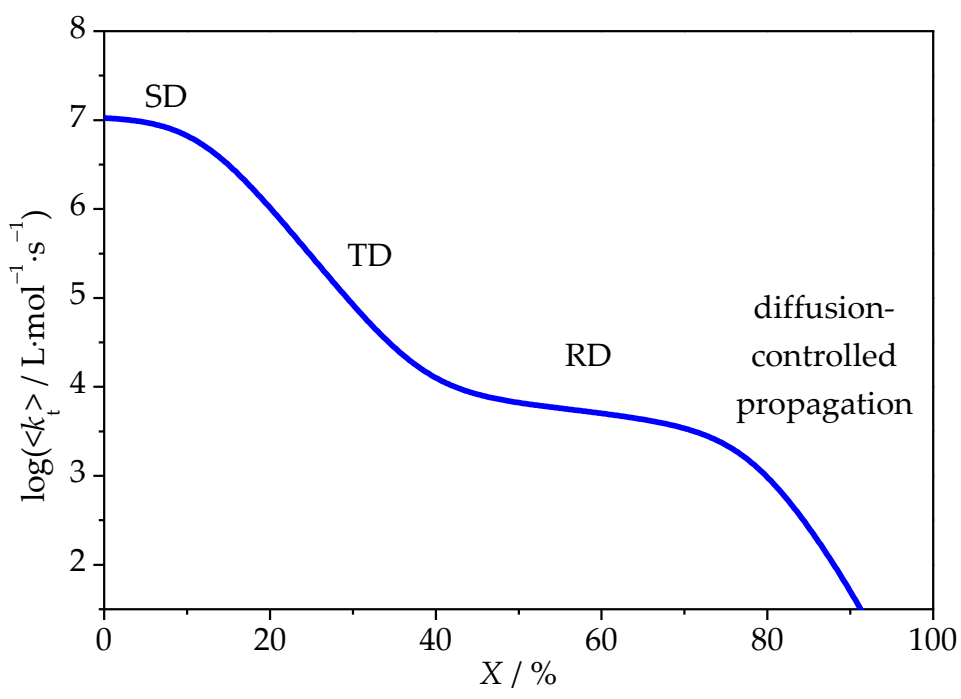

Figure 2.3: Conversion dependence of $\left\langle k_{t}\right\rangle$ for methyl methacrylate in bulk at $50{ }^{\circ} \mathrm{C}$ taken from literature. ${ }^{[62]}$

region, $\left\langle k_{t}\right\rangle$ decreases less than before. Here, the diffusion of macroradicals is more restricted because of the entanglement of the macroradicals, and hence the radical chain ends of the macroradicals can only approach each other by propagation. This is called reaction diffusion (RD) and the lesser decrease of $\left\langle k_{t, R D}\right\rangle$ is obtained by $k_{\mathrm{p}}$ and the reactiondiffusion constant $C_{\mathrm{RD}}$ by the ensuring equation: ${ }^{[62]}$

$$
\left\langle k_{\mathrm{t}, \mathrm{RD}}\right\rangle=k_{\mathrm{p}} \cdot C_{\mathrm{RD}} \cdot(1-X) .
$$

In the last region, $\left\langle k_{t}\right\rangle$ decreases again significantly because the propagation becomes diffusion controlled. Describing $\left\langle k_{\mathrm{t}}\right\rangle$ in general, the equations of the diffusion-controlled propagation and termination are combined to Equation 2.14. ${ }^{[62]}$ 


$$
\left\langle k_{\mathrm{t}}\right\rangle=\left\langle k_{\mathrm{t}, \mathrm{d}}\right\rangle+\left\langle k_{\mathrm{t}, \mathrm{RD}}\right\rangle=\frac{1}{\frac{1}{\left\langle k_{\mathrm{t}, \mathrm{SD}}\right\rangle}+\frac{\eta_{\mathrm{r}}}{\left\langle k_{\mathrm{t}, \mathrm{TD}}^{0}\right\rangle}}+\frac{C_{\mathrm{RD}} \cdot(1-X)}{\frac{1}{k_{\mathrm{p}}^{0}}+\frac{\eta_{\mathrm{r}}(X)}{k_{\mathrm{p}, \mathrm{d}}^{0}}}
$$

However, the different regions and the relating rate-determining diffusion processes depend on the monomer.

\subsection{Chain-Length Dependency of Radical Homopolymerizations}

The chain length of the macroradicals increase significantly during a radical polymerization. Thus, the chain length of macroradicals affects the propagation and termination rates which is explained in the following parts.

\subsubsection{Chain-Length Dependency of Propagation}

Although the propagation is chemically controlled up to high monomer-to-polymer conversion (see Chapter 2.3.2), experiments showed an exponential decrease of $k_{\mathrm{p}}$ at small chain lengths $(i<10) \cdot{ }^{[65]}$ For $i>10, k_{\mathrm{p}}$ is adequately represented by the long-chain propagation rate coefficient $k_{\mathrm{p}}^{\infty}{ }^{[66,67]}$ This behavior was explained by the reduction of the local monomer concentration at the radical chain end caused by the increasing chain length. ${ }^{[67]}$ Furthermore, theoretical treatment of experimental data led to the following equation for the chain-length dependent propagation rate coefficient $k_{\mathrm{p}}^{i}$ : $^{[68]}$

$$
k_{\mathrm{p}}^{i}=k_{\mathrm{p}}^{\infty}\left[1+C_{1} \exp \left(-\frac{\ln 2}{i_{1 / 2}}(i-1)\right)\right] .
$$

The magnitude of chain-length dependence is given by $i_{1 / 2}$ which can be understood as "half-life" of first order kinetics. The decrease of $k_{\mathrm{p}}$ is obtained by $C_{1}$ as follows: ${ }^{[68]}$

$$
C_{1}=\frac{k_{\mathrm{p}}^{1}-k_{\mathrm{p}}^{\infty}}{k_{\mathrm{p}}^{\infty}}
$$

where $k_{\mathrm{p}}^{1}$ is the initial propagation rate coefficient. Further explanation for the chain-length dependent propagation was found by using the 
transition state theory where the decrease of the pre-exponential factor $A_{0}$ with increasing chain length was observed due to a more hindered transition state. ${ }^{[69]}$

\subsubsection{Chain-Length Dependency of Termination}

Regarding Equation 2.7, the hydrodynamic radii of macroradicals increase with increasing chain length, and thus their diffusion becomes slower. Moreover, macroradicals with different chain lengths are formed during a radical polymerization. Therefore, three models with different weighting of the chain length are commonly used for describing the chain-length dependent termination rate coefficient $k_{t}^{i, j}$ of two macroradicals with the chain length $i$ and $j$ (Equation 2.17-2.19). ${ }^{[57,70]}$ The harmonic mean model $(\mathrm{hmm})$ is the most accurate description if the entanglement of the radical chain ends is rate-determining. For small molecules where the translational diffusion is the dominant diffusion process, the diffusion mean model $(\mathrm{dmm})$ can be used. The geometric mean value (gmm) has no physical meaning but can be applied for the termination rate coefficient if the segmental diffusion is rate determining. ${ }^{[5]}$

$$
\begin{gathered}
\mathrm{hmm}: k_{\mathrm{t}}^{i, j}=k_{\mathrm{t}}^{1,1} \cdot\left(\frac{2 \cdot i \cdot j}{i+j}\right)^{-\alpha} \\
\mathrm{dmm}: k_{\mathrm{t}}^{i, j}=0.5 \cdot k_{\mathrm{t}}^{1,1} \cdot\left(i^{-\alpha}+j^{-\alpha}\right) \\
\mathrm{gmm}: k_{\mathrm{t}}^{i, j}=k_{\mathrm{t}}^{1,1} \cdot(\sqrt{i \cdot j})^{-\alpha}
\end{gathered}
$$

The exponent $\alpha$ expresses the strength of the chain-length dependency and $k_{\mathrm{t}}^{1,1}$ is the termination rate coefficient of two monomeric radicals. Furthermore, if only macroradicals with the identical chain-length terminate, these models can be simplified to the following equation:

$$
k_{\mathrm{t}}^{i, i}=k_{\mathrm{t}}^{1,1} \cdot i^{-\alpha} .
$$

However, this equation does not consider the change of the dominating diffusion process with increasing chain length. Therefore, the Composite Model was developed by Smith, Russel and Heuts. ${ }^{[2]}$ In this 


\subsection{Chain-Length Dependency of Radical Homopolymerizations}

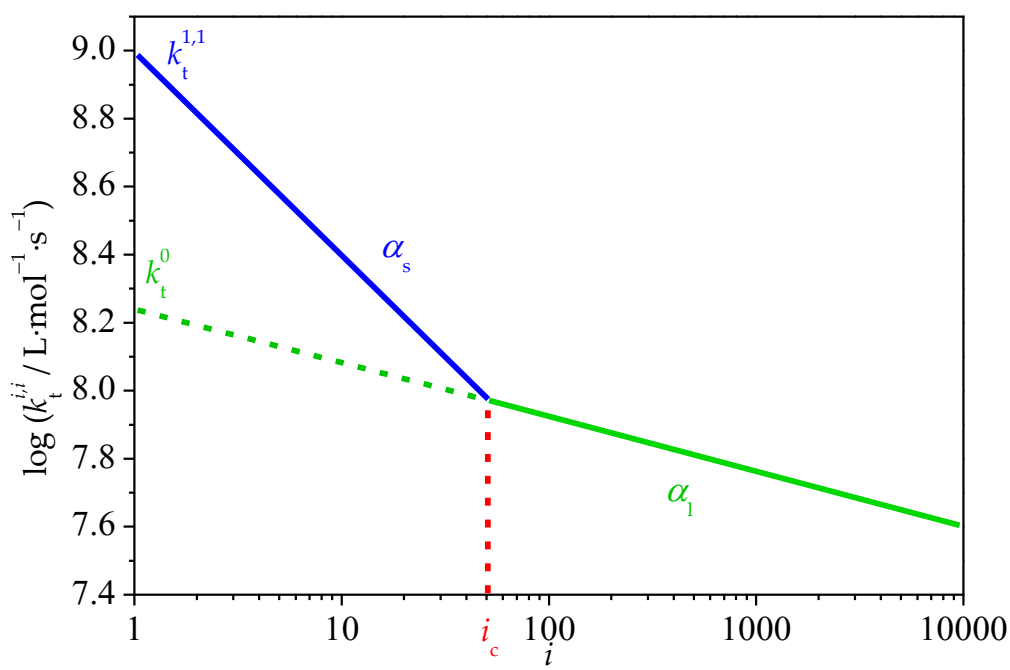

Figure 2.5: Chain-length dependence of $k_{\mathrm{t}}^{i, i}$ described by the Composite Model with $i_{\mathrm{c}}=50, \alpha_{\mathrm{s}}=0.6, \alpha_{\mathrm{l}}=0.16, k_{\mathrm{t}}^{1,1}=1 \cdot 10^{9} \mathrm{~L} \cdot \mathrm{mol}^{-1} \cdot \mathrm{s}^{-1}$.

model, two regions are proposed to describe $k_{\mathrm{t}}^{i, i}$ as depicted exemplary in Figure 2.5. In the short-chain region, the dominant diffusion process is the translation diffusion and $k_{\mathrm{t}}^{i, i}$ is described by Equation 2.21.

$$
k_{\mathrm{t}}^{i, i}=k_{\mathrm{t}}^{1,1} \cdot i^{-\alpha_{\mathrm{s}}} \text { with } i \leq i_{\mathrm{c}}
$$

Here, $\alpha_{\mathrm{s}}$ is the exponent for the short-chain region. After reaching the crossover chain length $i_{\mathrm{c}}$ (red line in Figure 2.5), the macroradicals are long enough (long-chain region) to entangle, and hence the termination is dominated by the segmental diffusion of two entangled macroradicals (Equation 2.22). ${ }^{[29]}$ Thus, with $\alpha_{1}$ as exponent for the long-chain region and $k_{\mathrm{t}}^{0}$ as termination rate coefficient of two hypothetical coiled monomeric radicals, $k_{\mathrm{t}}^{i, i}$ for long macroradicals is obtained by the Composite Model as follows:

$$
k_{\mathrm{t}}^{i, i}=k_{\mathrm{t}}^{1,1} \cdot i_{\mathrm{c}}^{-\alpha_{\mathrm{s}}+\alpha_{1}} \cdot i^{-\alpha_{1}}=k_{\mathrm{t}}^{0} \cdot i^{-\alpha_{1}} \text { with } i>i_{\mathrm{c}} .
$$


Moreover, the diffusion rate coefficient $k_{\mathrm{D}}$ of small molecules is adequately described by the Smoluchowski equation (Equation 2.23). Hence, this equation can also be assumed for $k_{\mathrm{t}}{ }^{1,1} \cdot{ }^{[71]}$

$$
k_{\mathrm{D}}=k_{\mathrm{t}}^{1,1}=4 \cdot \pi \cdot P_{\text {Spin }} \cdot N_{\mathrm{A}} \cdot\left(D_{\mathrm{A}}+D_{\mathrm{B}}\right) \cdot R_{\mathrm{c}}
$$

Here, $N_{\mathrm{A}}$ is the Avogadro constant, $P_{\text {Spin }}$ the spin factor and $R_{\mathrm{c}}$ the capture radius. In combination with the Stokes-Einstein equation (Equation 2.7), $D_{\mathrm{A}}=D_{\mathrm{B}}$ and $P_{\text {Spin }}=0.25,{ }^{[72]}$ the following equation is obtained:

$$
k_{\mathrm{t}}^{1,1}=\frac{R \cdot T \cdot R_{\mathrm{c}}}{6 \cdot \eta \cdot r_{1}} .
$$

Furthermore, this equation can be simplified with the assumption of spherical radicals $R_{\mathrm{c}}=2 r_{1}$ to the so-called "diffusion limit" (Equation 2.25), and thus $k_{\mathrm{t}}^{1,1}$ can be directly related to the fluidity $\eta^{-1}$. The "diffusion limit" represents the limit of $k_{\mathrm{t}}^{1,1}$ at a certain temperature and viscosity.

$$
k_{\mathrm{t}}^{1,1}=\frac{R \cdot T}{3 \cdot \eta}
$$

\subsection{Kinetics of Radical Copolymerizations}

The described kinetic schemes in the chapter above are only true if only one type of monomer is polymerized (homopolymerization). However, the kinetics are increasingly more difficult to describe in case of a copolymerization. Some aspects of the homopolymerization are transferrable to the radical copolymerization. A radical copolymerization can thus be separated in initiation, propagation and termination. However, as might be expected, the two or more types of monomers lead to complex kinetics of radical copolymerization. For each reaction step, the rate coefficients of the homopolymerizations can be exchanged by the average rate coefficients over the different compositions of copolymers. Moreover, beside the diffusion control and chain-length dependency further aspects must be considered. The formed macroradicals and also the formed copolymer differs in their compositions, sequence distribution and radical chain ends. ${ }^{[5]}$ All this leads to complex kinetics 
Table 2.1: Copolymerization categories depending on the copolymerization parameters and the product of those. ${ }^{[73]}$

\begin{tabular}{cccc}
\hline & copolymerization parameter & Product \\
& $r_{a}$ & $r_{b}$ & $r_{a} \cdot r_{b}$ \\
\hline $\begin{array}{c}\text { alternating } \\
\text { alternating, not } \\
\text { azeotropic }\end{array}$ & 0 & 0 & 0 \\
statistic, azeotropic & $0<r_{a}<1$ & $0<r_{b}<1$ & 0 \\
ideal & 1 & 1 & $<1$ \\
statistic, not azeotropic & $0<r_{a}<1$ & $1<r_{b}<\infty$ & $<1$ or $>1$ \\
block building & $1<r_{a}<\infty$ & $1<r_{b}<\infty$ & $>1$ \\
blend building & $\infty$ & $\infty$ & $>1$ \\
\hline
\end{tabular}

for radical copolymerization. Therefore, different copolymerization models were developed and the most important models will be further explained..$^{[5]}$

Moreover, copolymerizations can be divided in different categories depending on the copolymerization parameters (Table 2.1). The copolymerization parameters are explained in Chapter 2.5.2.1. Interesting for SP-PLP-ERP measurements is the statistic azeotropic copolymerization because at the azeotropic point the copolymerization is independent from the composition drift.

\subsubsection{Initiation of Radical Copolymerizations}

It can be assumed that the decomposition of the initiator is independent from the composition of the copolymer. However, it might be that the primary radicals prefer one monomer to the other. ${ }^{[74]}$

\subsubsection{Propagation of Radical Copolymerizations}

More important is the propagation kinetics of a radical copolymerization because it influences the composition, sequence 
distribution and the chain ends. ${ }^{[5]}$ The propagation kinetics of radical copolymerizations is very complex, shown by the countless models, which were developed for the propagation of copolymerizations. A good overview of these models is given in the Handbook of Radical Polymerizations. ${ }^{[5]}$ Subsequently, only the two models that are used throughout this thesis will be expounded.

\subsubsection{Terminal Model}

In 1944, Mayo and Lewis, ${ }^{[75]}$ and Alfrey and Goldfinger, ${ }^{[76]}$ independently developed the so-called terminal model. In this model, it is assumed that only the terminal unit of a macroradical, this means the radical chain end, influences the propagation kinetics of a copolymerization. Thus, four different propagation rate coefficients are necessary for describing the propagation kinetics of copolymerizations (see Figure 2.6). With this terminal model, the copolymer composition $\frac{F_{a}}{F_{b}}$ is obtained by the following equation:

$$
\frac{F_{a}}{F_{b}}=\frac{f_{a}}{f_{b}} \cdot \frac{r_{a} f_{a}+f_{b}}{r_{b} f_{b}+f_{a}} \text { with } a, b=1 \text { or } 2,
$$

where $f_{a}$ is the monomer feed fraction of monomer $a$ and $r_{a}$ reactivity ratios or also known as copolymerization parameter which is the ratio of the homo-propagation rate coefficient $k_{\mathrm{p}, a a}$ and the cross-propagation rate coefficient $k_{\mathrm{p}, a b}$ (Equation 2.27).

$$
r_{a}=\frac{k_{\mathrm{p}, a a}}{k_{\mathrm{p}, a b}} \text { with } a, b=1 \text { or } 2
$$

In Figure 2.7, the copolymerization diagram for styrene (sty) and methyl methacrylate (MMA) in bulk at $40{ }^{\circ} \mathrm{C}$ taken from the literature is shown. ${ }^{[50]}$ The terminal model (red line in Figure 2.7) can describe the composition adequately. Thus, the terminal model was also assumed to describe the average propagation rate coefficient of copolymerization $k_{\mathrm{p}, \text { copo }}$ correctly by Equation 2.28. ${ }^{[50]}$ 


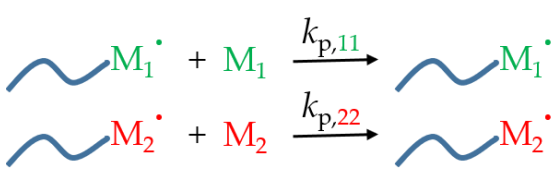

homo-propagation

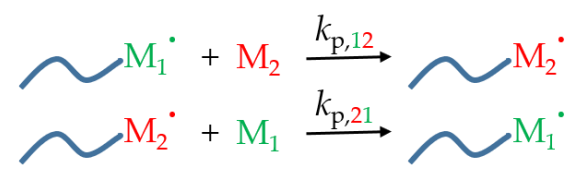

cross-propagation

Figure 2.6: Schematic illustration of the propagation steps of a radical copolymerization with two different monomers $\mathrm{M}_{1}$ and $\mathrm{M}_{2}$ if only the terminal unit influences the propagation kinetics (terminal model).

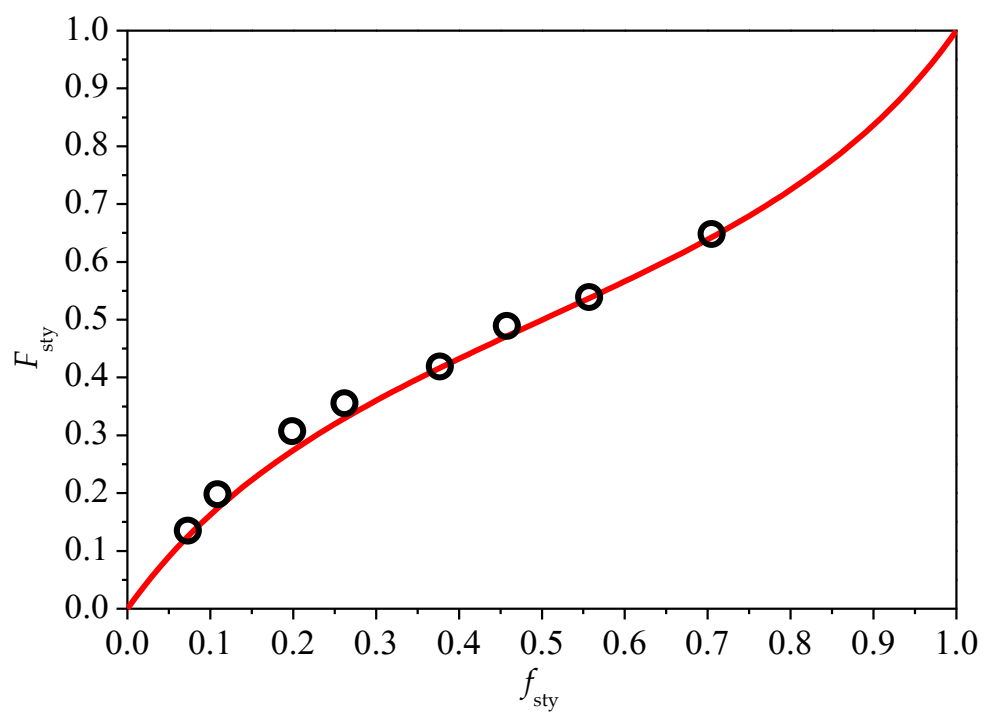

Figure 2.7: Copolymerization diagram for styrene and MMA in bulk at $40{ }^{\circ} \mathrm{C}$. The molar feed fraction of styrene $f_{\text {sty }}$ and molar fraction of styrene in the copolymer $F_{\text {sty }}$ was taken from literature. ${ }^{[50]}$ The red line is the fit obtained via the terminal model with $r_{\text {sty }}=0.523$ and $r_{\mathrm{MMA}}=0.460 .{ }^{[50]}$

$$
k_{\mathrm{p}, \mathrm{copo}}=\frac{r_{a} f_{a}^{2}+2 f_{a} f_{b}+r_{b} f_{b}^{2}}{\frac{r_{a} f_{a}}{k_{\mathrm{p}, a a}}+\frac{r_{b} f_{b}}{k_{\mathrm{p}, a b}}} \text { with } a, b=1 \text { or } 2
$$

However, for some copolymerization systems such as styrene and acrylonitrile the terminal model failed to describe the composition. ${ }^{\text {[7] }}$ 
Therefore, other models had to be taken into account for copolymerizations.

\subsubsection{Penultimate Model}

With the upcoming of accurate measurements of propagation rate coefficients (rotating sector, PLP-SEC), Fukuda found that the terminal model is not able to describe the real behavior of the average propagation rate coefficient for the copolymerization system of styrene and MMA (see red line Figure 2.8). ${ }^{[50]}$ Therefore, Fukuda used the penultimate model which was developed by Merz, Alfrey and Goldfinger in 1946. ${ }^{[78]}$ In this model, it is expected that terminal and penultimate units influence the propagation kinetics of copolymerizations. This results in eight different rate coefficients (Figure 2.9). For the penultimate unit model, the reactivity ratios of the monomers (Equation 2.29 and 2.30) and the reactivity ratio of the radicals (Equation 2.31) must be considered.

$$
\begin{aligned}
& r_{a}=\frac{k_{\mathrm{p}, a a a}}{k_{\mathrm{p}, a a b}} \text { with } a, b=1 \text { or } 2 \\
& r_{a}{ }^{\prime}=\frac{k_{\mathrm{p}, b a a}}{k_{\mathrm{p}, b a b}} \text { with } a, b=1 \text { or } 2 \\
& \mathrm{~s}_{a}=\frac{k_{\mathrm{p}, b a a}}{k_{\mathrm{p}, a a a}} \text { with } a, b=1 \text { or } 2
\end{aligned}
$$

Under consideration of the penultimate unit effect, the equations for copolymer composition (Equation 2.32) and for average propagation rate coefficient (Equation 2.33) become more complex. ${ }^{[50]}$

$$
\begin{gathered}
\frac{F_{a}}{F_{b}}=\frac{f_{a}}{f_{b}} \cdot \frac{\bar{r}_{a} f_{a}+f_{b}}{\bar{r}_{b} f_{b}+f_{a}} \text { with } a, b=1 \text { or } 2 \\
k_{\mathrm{p}, \text { copo }}=\frac{\bar{r}_{a} f_{a}^{2}+2 f_{a} f_{b}+\bar{r}_{b} f_{b}^{2}}{\bar{r}_{a} f_{a}} \overline{\bar{r}}_{b} f_{b} \\
\bar{k}_{\mathrm{p}, a a}
\end{gathered}
$$




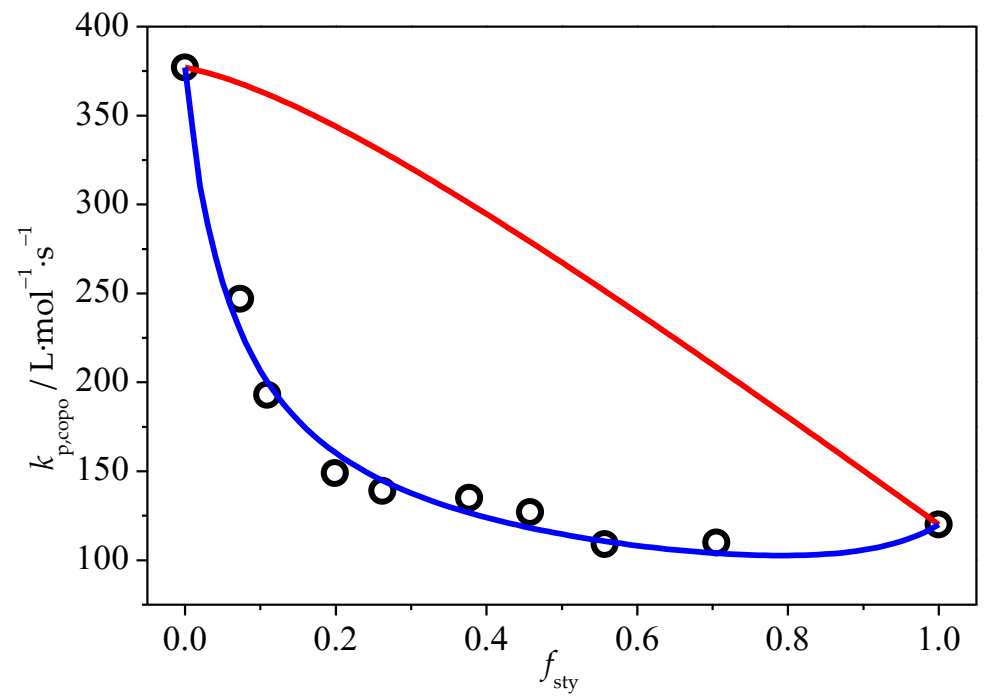

Figure 2.8: Experimental data for $k_{\mathrm{p}, \text { copo }}$ for different monomer feed fractions of styrene $f_{\text {sty }}$ for the copolymerization of styrene and MMA in bulk at $40{ }^{\circ} \mathrm{C}$ taken from literature. ${ }^{[50]}$ The red line represents the description by the terminal model with $r_{\text {sty }}=0.523$ and $r_{\mathrm{MMA}}=0.460$. The blue line represents the description by the implicit penultimate model with $r_{\text {sty }}=0.523, r_{\mathrm{MMA}}=0.460, s_{\text {sty }}=0.30$ and $s_{\mathrm{MMA}}=0.53 .{ }^{[50]}$

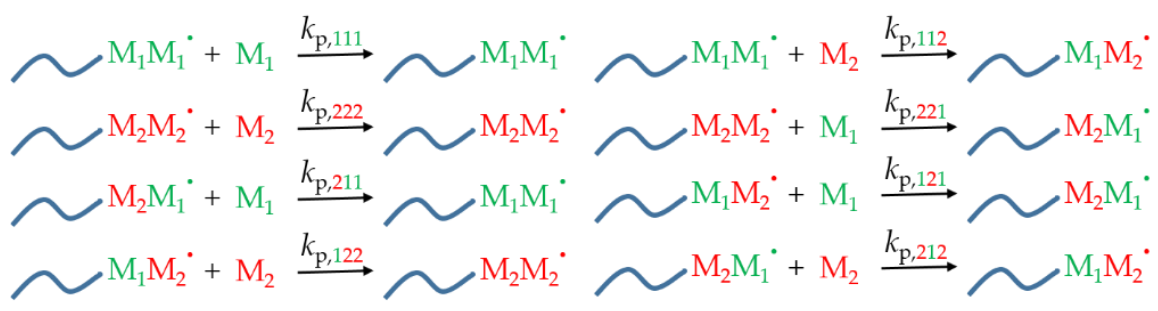

Figure 2.9: Schematic illustration of the propagation steps of a radical copolymerization with two different monomers $\mathrm{M}_{1}$ and $\mathrm{M}_{2}$ if terminal and penultimate units influence the propagation kinetics (penultimate model). 


$$
\begin{gathered}
\bar{r}_{a}=r_{a}{ }^{\prime}\left(\frac{r_{a} f_{a}+f_{b}}{r_{a}{ }^{\prime} f_{a}+f_{b}}\right) \text { with } a, b=1 \text { or } 2 \\
\bar{k}_{\mathrm{p}, a a}=k_{\mathrm{p}, a a a}\left(\frac{r_{a} f_{a}+f_{b}}{r_{a} f_{a}+\frac{f_{b}}{s_{a}}}\right) \text { with } a, b=1 \text { or } 2 .
\end{gathered}
$$

This model is also known as the explicit penultimate model. With the six adjustable copolymerization parameters, the explicit penultimate model is quite complex. Considering that the terminal model describes the composition of a copolymerization sufficiently, Fukuda simplified the penultimate model by assuming $r_{a}=r_{a}{ }{ }^{[79,80]}$ Thus, the penultimate unit only affects the reactivity but not the selectivity. As shown as the blue line in Figure 2.8, the implicit penultimate model represents experimental data for $k_{\mathrm{p}, \text { copo }}$ quite well. However, it should be noted that the multiple adjustable parameters lead to the good fit. Hence, it was not easy to find out whether the implicit or explicit model should be used. Therefore, theoretical calculations ${ }^{[81-83]}$ were performed and it could be verified that the explicit penultimate model should be used instead of the implicit penultimate model. Moreover, the existence of the penultimate unit effect (PUE) was observed during EPR experiments. ${ }^{[84,85]}$ Nevertheless, even the penultimate model may fail to describe the propagation kinetics for some copolymerization, for instance the copolymerization of styrene and methyl ethacrylate. ${ }^{[86]}$

\subsubsection{Termination of Radical Copolymerizations}

Just like the propagation, the termination of a radical copolymerization is complicated, and models are necessary for describing it. Walling introduced the cross-termination factor $\Phi$ which is given by the following equation: ${ }^{[87]}$

$$
\Phi=\frac{\left\langle k_{\mathrm{t}, a b}\right\rangle}{\left(\left\langle k_{\mathrm{t}, a \mathrm{a}}\right\rangle\left\langle k_{\mathrm{t}, b b}\right\rangle\right)^{0.5}} \text { with } a, b=1 \text { or } 2,
$$


where $\left\langle k_{\mathrm{t}, a b}\right\rangle$ is the cross-termination rate coefficient and $\left\langle k_{\mathrm{t}, a a}\right\rangle$ and $\left\langle k_{\mathrm{t}, b b}\right\rangle$ the homo-termination rate coefficients. This factor quantifies the preference for cross-termination compared to homo-termination. Because this model depends on the chemical structure of the terminal unit of the macroradicals, a chemical control is assumed for termination. As shown in Chapter 2.3.3, this is not the case for homopolymerizations, and it should also not be the case for copolymerizations. Under consideration of diffusion-controlled termination, the "ideal" diffusion model was developed, in which the homo-termination rate coefficients are linearly combined by the mole fraction of the monomer in the copolymer. ${ }^{[8]}$

$$
\left\langle k_{\mathrm{t}, \text { copo }}\right\rangle=F_{a}\left\langle k_{\mathrm{t}, a a}\right\rangle+F_{b}\left\langle k_{\mathrm{t}, b b}\right\rangle \text { with } a, b=1 \text { or } 2
$$

Another reasonable description is using the inverse rate coefficient (Equation 2.38). ${ }^{[89]}$

$$
\left\langle k_{\mathrm{t}, \mathrm{copo}}\right\rangle^{-1}=F_{a}\left\langle k_{\mathrm{t}, a a}\right\rangle^{-1}+F_{b}\left\langle k_{\mathrm{t}, b b}\right\rangle^{-1} \text { with } a, b=1 \text { or } 2 .
$$

Moreover, it is also possible to consider the terminal model as an important factor for the termination kinetics of copolymerizations. With the relative radical concentration $P_{a}$ with monomer $a$ as terminal unit, $\left\langle k_{\mathrm{t}, \text { copo }}\right\rangle$ can be expressed as follows: ${ }^{[90]}$

$$
\left\langle k_{\mathrm{t}, \mathrm{copo}}\right\rangle=\sum_{a=1}^{2} \sum_{b=1}^{2} P_{a} P_{b}\left\langle k_{\mathrm{t}, a b}\right\rangle .
$$

If the segmental diffusion is rate-determining, it is better to implement the penultimate unit into the termination model (Equation 2.40). ${ }^{[90]}$

$$
\left\langle k_{\mathrm{t}, \mathrm{copo}}\right\rangle=\sum_{a=1}^{2} \sum_{b=1}^{2} \sum_{c=1}^{2} \sum_{d=1}^{2} P_{a b} P_{d e}\left\langle k_{\mathrm{t}, a b, d e}\right\rangle
$$

Furthermore, this penultimate termination model can be simplified by the by geometric mean value (Equation 2.41) or algebraic mean value (Equation 2.42). ${ }^{[90]}$ 


$$
\begin{aligned}
\left\langle k_{\mathrm{t}, \mathrm{copo}}\right\rangle^{0.5} & =\left\langle k_{\mathrm{t} 11,11}^{0.5}\right\rangle P_{11}+\left\langle k_{\mathrm{t} 12,12}{ }^{0.5}\right\rangle P_{12}+\left\langle k_{\mathrm{t} 21,21}{ }^{0.5}\right\rangle P_{21} \\
& +\left\langle k_{\mathrm{t} 22,22}{ }^{0.5}\right\rangle P_{22} \\
\left\langle k_{\mathrm{t}, \text { copo }}\right\rangle= & \left\langle k_{\mathrm{t} 11,11}\right\rangle P_{11}+\left\langle k_{\mathrm{t} 12,12}\right\rangle P_{12}+\left\langle k_{\mathrm{t} 21,21}\right\rangle P_{21}+\left\langle k_{\mathrm{t} 22,22}\right\rangle P_{22}
\end{aligned}
$$

However, none of these models considers the chain-length dependency of termination as explained in detail for homopolymerizations in Chapter 2.4.2. Therefore, first investigations into the chain-length dependent termination of copolymeirzations were performed by Olaj et al. ${ }^{[53]}$ In this study, $k_{\mathrm{t} \text {, copo }}^{1,1}$ was determined for the copolymerization of styrene and MMA and surprisingly $k_{\mathrm{t}, \mathrm{copo}}^{1,1}$ was significantly higher than the termination rate coefficients $k_{t}^{1,1}$ for the relating homopolymerizations. This unexpected behavior was explained by the increasing mobility caused by the alternation tendency of the copolymerization of styrene and MMA, and thus $\varphi$ as a quantity for chain flexibility was introduced (Equation 2.43). ${ }^{[53]}$

$$
k_{\mathrm{t}, \text { copo }}^{1,1}=f_{1}^{2} k_{\mathrm{t}, 11}^{1,1}+2 \varphi f_{1} f_{2} \overline{k_{\mathrm{t}}^{1,1}}+f_{2}^{2} k_{\mathrm{t}, 22}^{1,1}
$$

However, this is the only investigation into the chain-length dependency of termination in copolymerizations so far and it is not clear whether this prediction is correct. ${ }^{[53]}$

\subsection{SP-PLP-EPR Method}

For investigations into the chain-length dependent termination kinetics, the powerful SP-PLP-EPR method was developed by Buback and coworkers. ${ }^{[25-27]}$ After instantaneous initiation of pulsed-laser polymerization (PLP) by a single laser pulse (SP), the decay of the radical concentration is detected time-resolved via electron paramagnetic resonance (EPR) spectroscopy. Because of the instantaneous initiation, it can be assumed that only macroradicals with the identical chain length $i$ terminate after applying the single pulse. If no transfer reaction such as backbiting occurs, $i$ can be correlated to the time $t$ (Equation 2.44). 


$$
i=c_{\mathrm{M}} \cdot k_{\mathrm{p}} \cdot t=\frac{t}{t_{\mathrm{p}}}
$$

After implementation of Equation 2.44 in the rate law for termination, integration and insertion of the Composite Model (Equation 2.22 and 2.23), the following equations are obtained:

$$
\begin{aligned}
& \frac{c_{\mathrm{R}}^{0}}{c_{\mathrm{R}}(t)}-1=\frac{2 \cdot k_{\mathrm{t}}^{1,1} \cdot c_{\mathrm{R}}^{0} \cdot t_{\mathrm{p}}^{\alpha_{\mathrm{s}}}}{1-\alpha_{\mathrm{s}}} t^{1-\alpha_{\mathrm{s}}} \text { with } t \leq t_{\mathrm{c}} \\
& \frac{c_{\mathrm{R}}^{0}}{c_{\mathrm{R}}(t)}-1=\frac{2 \cdot k_{\mathrm{t}}^{0} \cdot c_{\mathrm{R}}^{0} \cdot t_{\mathrm{p}}^{\alpha_{1}}}{1-\alpha_{1}} t^{1-\alpha_{1}} \text { with } t>t_{\mathrm{c}} .
\end{aligned}
$$

These equations can be easily linearized. Thus, the data from SP-PLPEPR is evaluated by a double logarithmic plot where short and long chain regions are linearly fitted. From the slope, $\alpha_{\mathrm{s}}$ and $\alpha_{1}$ can be determined and from the intersection of the two linear fits the crossover chain length $i_{\mathrm{c}}$ can be obtained by inserting $t_{\mathrm{c}}$ in Equation 2.44. Nevertheless, it turned out that this data treatment can result in systematic errors especially in the short chain region because the initiator fragment is not taken into account. Therefore, it was recommended to expand Equation 2.44 by addition of $1 .^{[91]}$ The so-obtained equation for evaluating the data from SP-PLP-EPR are given in Equation 2.47 and 2.48.

$$
\begin{gathered}
\frac{c_{\mathrm{R}}^{0}}{c_{\mathrm{R}}(t)}-1=\frac{2 \cdot k_{\mathrm{t}}^{1,1} \cdot c_{\mathrm{R}}^{0} \cdot\left[\left(c_{\mathrm{M}} \cdot k_{\mathrm{p}} \cdot t+1\right)^{1-\alpha_{\mathrm{s}}}-1\right]}{k_{\mathrm{p}} \cdot c_{\mathrm{M}} \cdot\left(1-\alpha_{\mathrm{s}}\right)} \text { with } t \leq t_{\mathrm{c}} \\
\frac{c_{\mathrm{R}}^{0}}{c_{\mathrm{R}}(t)}-1=\frac{2 \cdot k_{\mathrm{t}}^{1,1} \cdot c_{\mathrm{R}}^{0} \cdot\left[\left(i_{\mathrm{c}}\right)^{1-\alpha_{\mathrm{s}}}-1\right]}{k_{\mathrm{p}} \cdot c_{\mathrm{M}}\left(1-\alpha_{\mathrm{s}}\right)}-\frac{2 \cdot k_{\mathrm{t}}^{1,1} \cdot c_{\mathrm{R}}^{0} \cdot\left(i_{\mathrm{c}}\right)^{1-\alpha_{\mathrm{s}}}}{k_{\mathrm{p}} \cdot c_{\mathrm{M}} \cdot\left(1-\alpha_{1}\right)} \\
+\frac{2 \cdot k_{\mathrm{t}}^{1,1} \cdot c_{\mathrm{R}}^{0} \cdot\left[\left(c_{\mathrm{M}} \cdot k_{\mathrm{p}} \cdot \mathrm{t}+1\right)^{1-\alpha_{1}}-1\right]}{k_{\mathrm{p}} \cdot c_{\mathrm{M}}\left(1-\alpha_{1}\right)}
\end{gathered}
$$

These equations cannot be linearized and hence the composite parameter must be determined by an iterative fit. However, it turned out that it is difficult to treat the long chain region by this iterative fit. ${ }^{[32]}$ Therefore, it was recommended to use the iterative fit for evaluation of 
the short chain region and determine $\alpha_{1}$ and $i_{\mathrm{c}}$ by the double logarithmic plot. $^{[32]}$ 


\section{EXPERIMENTAL}

\subsection{Chemicals}

\subsubsection{Monomers}<smiles>C=C(C)C(=O)OC</smiles>

Methyl

methacrylate

(MMA, $M=100.12 \mathrm{~g} \cdot \mathrm{mol}^{-1}$, 99\%, Sigma-Aldrich) was purified by passing through a column filled with inhibitor remover (Sigma-Aldrich).<smiles>C=C(C)C(=O)OCCCCC</smiles>

Pentyl methacrylate

(PnMA, $\left.M=156.22 \mathrm{~g} \cdot \mathrm{mol}^{-1}, 95 \%, \mathrm{ABCR}\right)$, was purified by passing through a column filled with inhibitor remover (Sigma-Aldrich).<smiles>C=C(C)C(=O)OCC(CC)CCCC</smiles>

2-Ethylhexyl methacrylate (2-EHMA, $M=198.30 \mathrm{~g} \cdot \mathrm{mol}^{-1}, 98 \%$, Sigma-Aldrich) was purified by passing through a column filled with inhibitor remover (Sigma-Aldrich).<smiles>C=C(C)C(=O)OCCCCCCCC</smiles>

Dodecyl

methacrylate

(DMA, $M=254.41 \mathrm{~g} \cdot \mathrm{mol}^{-1}, 96 \%$, Sigma-Aldrich) was purified by passing through a column filled with inhibitor remover (Sigma-Aldrich).<smiles>C=Cc1ccccc1</smiles>

Styrene (Sty, $\quad M=104.15 \mathrm{~g} \cdot \mathrm{mol}^{-1}, \quad \geq 99 \%$, Sigma-Aldrich), was purified by passing through a column filled with aluminum oxide (neutral, Brockmann). 
<smiles>[2H]C([2H])=C([2H])c1c([2H])c([2H])c([2H])c([2H])c1[2H]</smiles>

Styrene-d8 (Sty-d8, $M=112.20 \mathrm{~g} \cdot \mathrm{mol}^{-1}$, 98\%, Cambridge Isotope Laboratories), was used as received.

\subsubsection{Photoinitiators}<smiles>CC(C)(OOC(C)(C)c1ccccc1)c1ccccc1</smiles>

Dicumyl peroxide (DCP, $M=270.37 \mathrm{~g} \cdot \mathrm{mol}^{-1}$, $98 \%$, Sigma-Aldrich) was used as received.<smiles>CSc1ccc(C(=O)C(C)(C)N2CCOCC2)cc1</smiles>

2-Methyl-4'-(methylthio)-2-morpholinopropiophenone (MMMP, $M=279.40 \mathrm{~g} \cdot \mathrm{mol}^{-1}$, $98 \%$, Sigma-Aldrich) was used as received.

\subsubsection{Calibration Agent}<smiles>CC1(C)CCCC(C)(C)N1[O]</smiles>

2,2,6,6-tetramethyl-1-piperidinyloxyl (TEMPO, $M=156.25 \mathrm{~g} \cdot \mathrm{mol}^{-1}, 98 \%$, Sigma-Aldrich) was used as received.

\subsection{EPR Measurements}

\subsubsection{Experimental Setup}

The used experimental setup is schematically illustrated in Figure 3.1. For the EPR measurements, a Bruker EPR CW/ transient spectrometer system Elexsys-II 500T, which operates in the X-Band (9-10 GHz) region, was used. The EPR spectrometer includes an ER 41122SHQE-LC cavity 


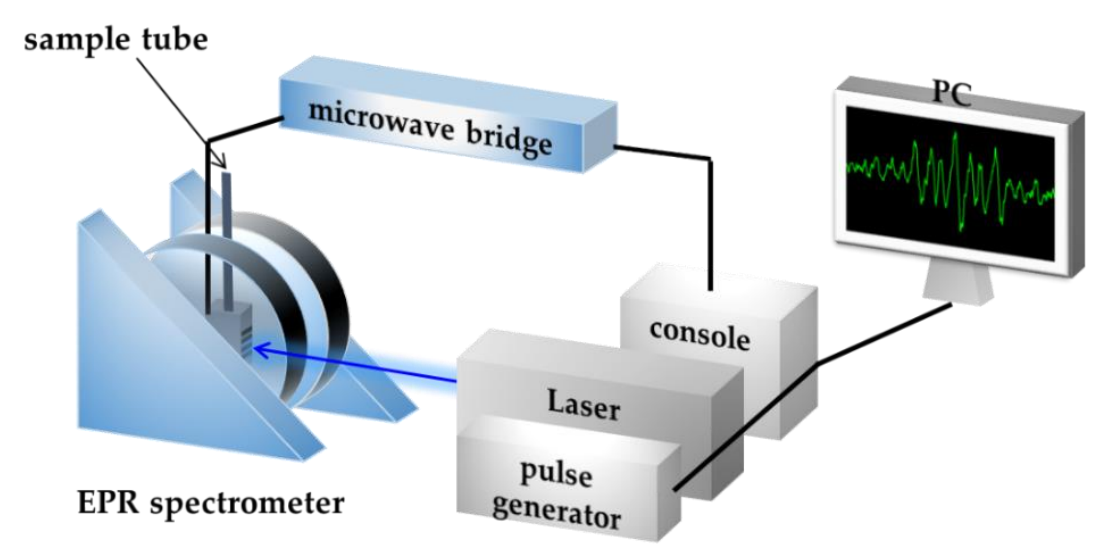

Figure 3.1: Schematic illustration of the used experimental setup including the EPR spectrometer which consists a cavity, magnets, microwave bridge and console, XeF laser or Nd:YAG laser, pulse generator and the connected PC. The telescope and shutter placed between spectrometer and laser are not depicted.

(Bruker). The sample tube is placed in this cavity so that the sample is completely irradiated. Therefore, the cavity is equipped with a grid as shown in Figure 3.2. The SP experiments of the copolymerization were performed with a XeF laser (LPX 210 iCC, Lambda Physik) operating at a wavelength of $351 \mathrm{~nm}$. Laser and spectrometer were synchronized by a Quantum Composers 9314 pulse generator (Scientific Instruments). However, the laser had to be replaced by a Nd:YAG laser (LPY774G-30, Nd-YAG, 3rd harmonic $355 \mathrm{~nm}$, Litron Lasers Ltd) which was used to investigate the termination kinetics of the homopolymerizations. Between laser and spectrometer, a telescope and a shutter were installed additionally. The telescope was necessary for homogenization of the laser beam. The shutter and EPR were synchronized by Quantum Composers 9314 pulse generator (Scientific Instruments) for the single pulse experiments.

The spectra were recorded under continuous irradiation with mercury-arc lamp (LAX 1450/SH2/5,500W, Müller) or under pseudostationary conditions using the laser with a pulse repetition rate (p.r.r.) of $30 \mathrm{~Hz}$. The temperature was controlled by an ER 4131VT unit (Bruker). Temperatures below room temperature were reached by evaporation of liquid nitrogen. To avoid condensation, the cavity was flushed with a continues flow of gaseous nitrogen. At temperatures above room 


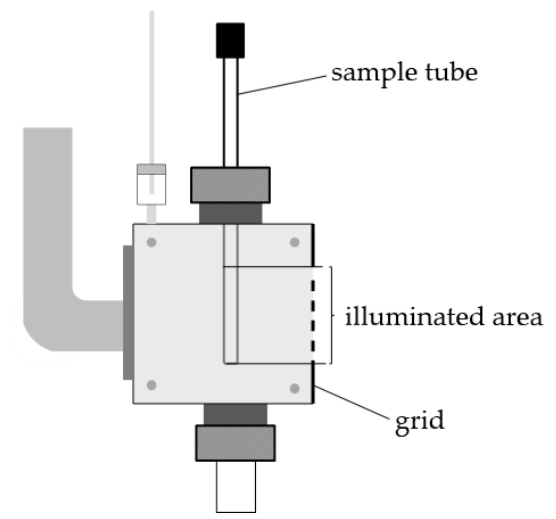

Figure 3.2: Schematic illustration of the cavity where the sample tube is placed in. Using the grid, a full irradiation of the sample is reached.

temperature, the cavity was flushed with a continuous flow of gaseous nitrogen to achieve a constant temperature. Thus, temperatures between $100 \mathrm{~K}$ and $600 \mathrm{~K}$ were accessible.

\subsubsection{Experimental Procedure}

A good signal-to-noise ratio was obtained using a modulation amplitude $3 \mathrm{G}$ and modulation frequency of $100 \mathrm{kHz}$, a receiver gain of 60 and an attenuation of $20 \mathrm{~dB}$ for homopolymerizations and $26 \mathrm{~dB}$ for copolymerizations, respectively.

\subsubsection{Sample Preparation}

With exception of styrene- $\mathrm{d} 8$, all monomers were degassed by several freeze-pump-thaw cycles under the exclusion of visible light with aluminum foil. Styrene-d8 was received in an ampule under argon atmosphere. Afterwards, the mixtures for copolymerization were prepared in a glove box under an argon atmosphere. The photoinitiator was added to the purified monomer and copolymerization mixture, respectively.

Depending on the polarity of the sample, different sample tubes were used. For investigations of high polar organic monomers (MMA, PnMA, 2-EHMA and copolymerization of styrene and MMA), $50 \mu \mathrm{L}$ of the monomer-photoinitiator solution was filled in EPR tubes (Wilmad, 
Table 3.1: $\quad$ The different copolymerization composition for styrene-d8 and MMA which were investigated.

\begin{tabular}{lllll}
\hline$f_{\text {sty-d8 }}$ & 0.118 & 0.239 & 0.349 & 0.467 \\
\hline$f_{\text {MMA }}$ & 0.881 & 0.761 & 0.651 & 0.533 \\
\hline
\end{tabular}

suprasil, $3 \mathrm{~mm}$ outer diameter, $2 \mathrm{~mm}$ inner diameter) using a microliter syringe ( $50 \mu \mathrm{L}$, Hamilton). Styrene and DMA are less polar, and thus $0.2 \mathrm{~mL}$ of these monomer-photoiniator solutions were filled in EPR tubes (Wilmad, suprasil, $5 \mathrm{~mm}$ outer diameter, $4 \mathrm{~mm}$ inner diameter) using an Eppendorf ${ }^{\circledR}$ pipette. However, styrene-d8 was measured in the smaller EPR tubes because of the small amount of styrene-d8. Afterwards, the EPR tubes were closed with a plastic cap and sealed with Parafilm ${ }^{\circledR}$.

All prepared sample tubes were excluded from light and measured immediately after preparation.

\section{Composition of Copolymerzations}

The different copolymerization compositions (Table 3.1) were prepared in a glovebox under argon atmosphere. The monomer feed fraction $f_{a}$ of monomer $a$ is given by the following equation:

$$
f_{a}=\frac{n_{a}}{n_{a}+n_{b}} \text {, with } a, b=1 \text { or } 2
$$

where $n_{a}$ is the amount of substance $a$. With a given volume, the density and molar mass for monomer $a, n_{a}$ can be determined whereas $n_{b}$ is obtained by Equation 3.2.

$$
n_{b}=1-n_{a} \text { with } a, b=1 \text { or } 2 .
$$

\subsubsection{Choice of Photoinitiator}

In particular, the photoinitiator is an important factor for SP-PLP-EPR measurements and therefore, it has to have the subsequent properties: ${ }^{[92]}$ 
(1) The initiator has to be thermally stable within the investigated temperature region.

(2) The initiator efficiency has to be high.

(3) The initiator has to absorb the applied wavelength-in this work $351 \mathrm{~nm}$ and $355 \mathrm{~nm}$, respectively.

(4) The decomposition of the initiator has to be faster than the initiation.

(5) The initiator has to be soluble in the monomer.

Studies have shown that MMMP perfectly fits into these criteria for some monomers. ${ }^{[92,93]}$ Thus, MMMP was used for the measurements of the DMA homopolymerizations. In termination kinetics study of vinyl acetate and vinyl pivalate, however, it has been observed that primary radicals of MMMP form a stable radical adduct (see Scheme 3.1). ${ }^{[94]}$ This is caused by the fact that vinyl and vinyl pivalate are more nucleophilic compared to monomers investigated in previous studies. Accordingly, MMMP cannot be used for investigations of those monomer types. Hence, the termination kinetics of the copolymerization of styrene and MMA were investigated with DCP as photoinitiator. It was successfully used for investigations into the termination kinetics of the homopolymerization of styrene and is known to initiate both types of monomers. ${ }^{[34,95]}$ However, during the investigations of PnMA within this thesis, a similar EPR Signal as observed in the literature ${ }^{[94]}$ occurred at low temperature $(233 \mathrm{~K})$ using MMMP as initiator (Figure 3.3). In contrast, using DCP as photoinitiator, such signals could not be observed during and after the irradiation with the laser. A possible reason for the formation of this stable radical is the changed laser type with slightly varied wavelength. Therefore, to avoid any effect of the stable MMMP radical on the termination kinetics, DCP was used for the investigations into the termination kinetics of PnMA and 2-EHMA. 
<smiles>CSc1ccc(C=O)cc1</smiles><smiles>CSc1ccc(C=O)cc1</smiles><smiles>CSc1ccc(C(=O)C(C)(C)N2CCOCC2)cc1</smiles><smiles>CSc1ccc(C(=O)OC(C)(C)N2CCOCC2)cc1</smiles>

Scheme 3.1: Decomposition of MMMP (top). Proposed mechanism of the formation of the stable radical adduct (bottom) in the literature. ${ }^{[94]}$

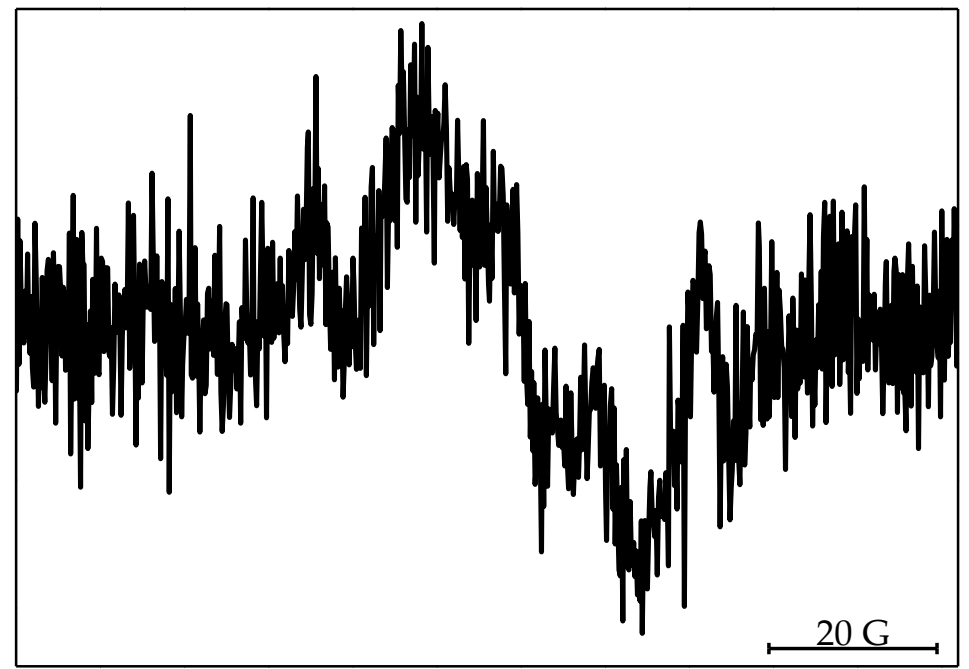

Figure 3.3: Spectrum after laser initiation of a PnMA homopolymerization with MMMP as photoinitiator in bulk at $233 \mathrm{~K}$. The spectrum was recorded without further irradiation.

The chosen concentration of photoinitiator differs for homopolymerizations and copolymerizations. For homopolymerizations, concentrations of $c_{\mathrm{MMMP}}=2.7 \cdot 10^{-3} \mathrm{~mol} \cdot \mathrm{L}^{-1}$ and 
$c_{\mathrm{DCP}}=9.0 \cdot 10^{-2} \mathrm{~mol} \cdot \mathrm{L}^{-1}$, respectively, were chosen. The chosen concentration of DCP was higher than this for MMMP because of the lower absorbance at $351 \mathrm{~nm}$ and $355 \mathrm{~nm}$ of DCP compared to MMMP. ${ }^{96]}$ In addition, it has to be considered that DCP affects the viscosity, and thus the termination kinetics. However, for the recorded copolymerization spectra, a higher concentration $\left(c_{\mathrm{DCP}}=0.5 \mathrm{~mol} \cdot \mathrm{L}^{-1}\right)$ was selected to improve the signal-to-noise ratio whereas for SP experiments of copolymerizations were conducted with $c_{\mathrm{DCP}}=9.0 \cdot 10^{-2} \mathrm{~mol} \cdot \mathrm{L}^{-1}$ to avoid a significant effect of the viscosity on the termination kinetics.

\subsubsection{Calibration}

The outstanding advantage of EPR is that absolute radical concentrations can be measured directly and time-resolved. To determine the absolute radical concentration, a two-step calibration is required ${ }^{[27]} \mathrm{In}$ the first step, the EPR spectra of a stable radical -in this work TEMPOwith at least three concentrations are measured. Therefore, a known concentration of TEMPO is solved in the monomer and copolymerization mixture, respectively. To minimize the errors, the calibration is carried out for several TEMPO concentrations under the same condition including the sample volume, sample tube, position of the tube in cavity and same temperature as for the SP-PLP-EPR experiments. Moreover, these spectra are recorded with the same measurement parameters (attenuation, receiver gain, sweep time) as for the SP-PLP-EPR experiments. Since the radical concentration $c_{R}(T E M P O)$ is proportional to the double integral of the intensity $\iint I(B)$ (Equation 3.3), the constant $h_{1}$ is determined from the slope of the linear fit of the corresponding plot (see Figure 3.4). An exemplary spectrum of TEMPO (blue), the integral of this $I(B)$ (green) and the double integral of this $\iint I(B)$ (black) is shown.

$$
c_{\mathrm{R}}(\mathrm{TEMPO})=h_{1} \cdot \iint I(B)
$$

However, a second calibration step is essential because the signal intensity is recorded during the SP experiments and not the double integral. In consequence, the double integral of the spectrum recorded during the polymerization has to be correlated to the intensity $I\left(B_{\max }\right)$ as follows: 


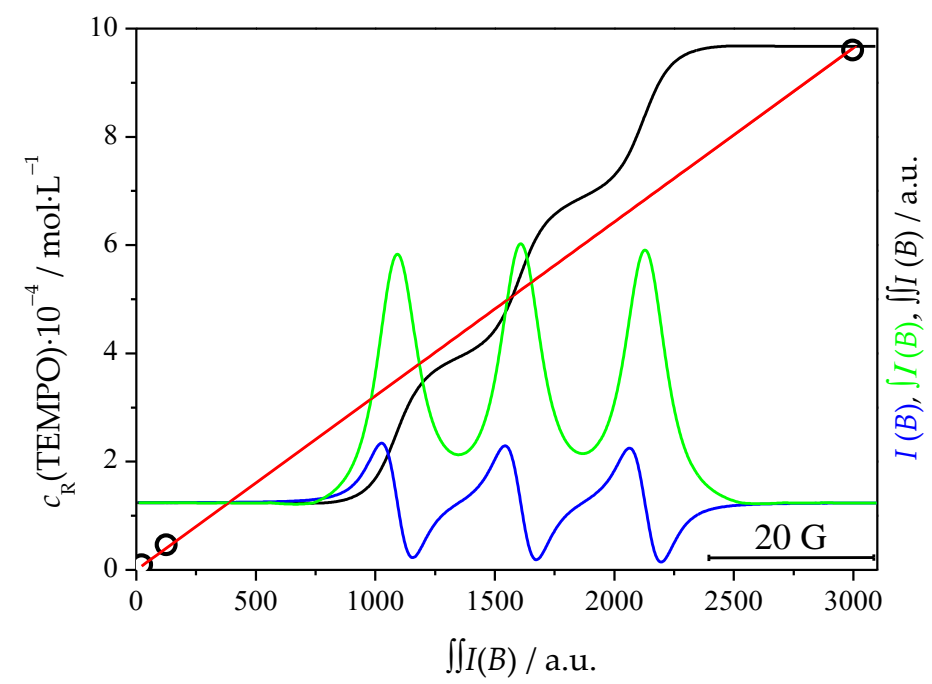

Figure 3.4: Plot of the radical concentration of TEMPO $c_{R}($ TEMPO) $\left(9.60 \cdot 10^{-4} \mathrm{~mol} \cdot \mathrm{L}^{-1}, 4.61 \cdot 10^{-5} \mathrm{~mol} \cdot \mathrm{L}^{-1}\right.$ and $\left.9.22 \cdot 10^{-6} \mathrm{~mol} \cdot \mathrm{L}^{-1}\right)$ against the double integral $\iint I(B)$ at $333 \mathrm{~K}$ for determination of the constant $h_{1}$. The TEMPO spectrum (blue) recorded for $c_{\mathrm{R}}(\mathrm{TEMPO})=9.60 \cdot 10^{-4} \mathrm{~mol} \cdot \mathrm{L}^{-1}$ and the related integrated (green) and double integrated (black) spectra are shown, too.

$$
\iint I(B)=h_{2} \cdot I\left(B_{\max }\right)
$$

By plotting $\iint I(B)$ against $I\left(B_{\max }\right)$, the constant $h_{2}$ is given by the slope of the linear fit (Figure 3.5). Thus, the absolute radical concentration at a certain $t$ is calculated by the subsequent equation:

$$
c_{\mathrm{R}}(t)=h_{1} \cdot h_{2} \cdot I\left(B_{\text {max }}, t\right) .
$$

\subsubsection{Determination of Conversion}

After the SP-PLP-EPR measurements, the conversion $X$ was determined gravimetrically. Therefore, an empty aluminum pan was weighted $\left(m_{1}\right)$. The monomer-photoinitiator solution was filled into the aluminum pan and weighted immediately $\left(m_{2}\right)$. Under ambient conditions and exclusion of light, the monomer evaporated, and 


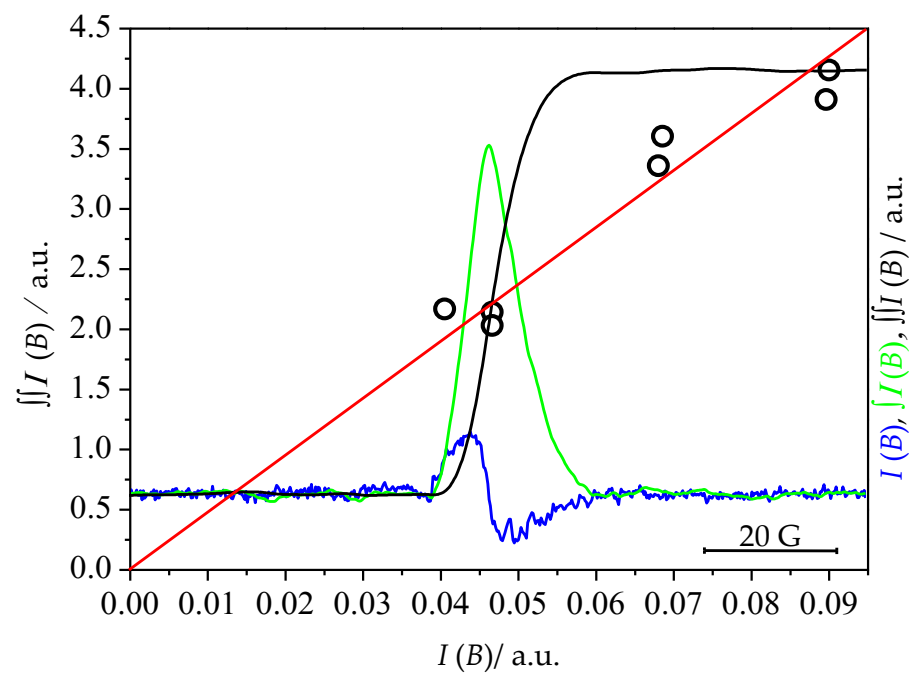

Figure 3.5: Plot of the double integral $\iint I(B)$ of the spectra recorded during the copolymerization of styrene-d8 and MMA $\left(f_{\text {sty- } 8}=0.498\right.$ and $\left.f_{\mathrm{MMA}}=0.502\right)$ at $333 \mathrm{~K}$ against intensity $I(B)$ for determination of the constant $h_{2}$. The copolymerization spectrum (blue) recorded and the related integrated (green) and double integrated (black) spectra are shown, too.

afterwards the aluminum pan was weighted again $\left(m_{3}\right)$. Thus, the conversion was determined by the following equation:

$$
X=\frac{m_{3}-m_{1}}{m_{2}-m_{1}} .
$$

\subsubsection{Density and Viscosity Measurements}

The density was determined with a density meter system DPR 2000 (Anton Paar) and the viscosity with a Viskosimeter AMVn (Anton Paar, 1569).

\subsubsection{Kinetic Simulations}

For kinetic simulations, the commercially available program PREDICI ${ }^{\circledR}$ was used. Thus, the concentration-time profile of the copolymerization of styrene-d 8 and MMA $\left(f_{\text {sty- } 8}=0.498\right.$ and $\left.f_{\mathrm{MMA}}=0.502\right)$ 
could be evaluated via parameter estimation. For the termination rate coefficients, the IUPAC recommended factor of 2 was considered. ${ }^{[59,60]}$

\subsubsection{Simulations of the EPR Spectra}

The EPR spectra were simulated with Easy Spin package for Matlab ${ }^{\circledR}$. In the Appendix, the used script for the copolymerization of styrene- $\mathrm{d} 8$ and MMA $\left(f_{\text {sty-d } 8}=0.118\right.$ and $\left.f_{\mathrm{MMA}}=0.881\right)$ is given. Beside the hyperfine coupling constants, the ratio of two or more coexisting radicals can be determined directly.

\subsection{Troubleshooting}

Within this work, some complications occurred during the SP-PLPEPR measurements. Some issues were caused by the replacements of some parts of the experimental setup and some by sample preparations. Solving these problems had a huge impact on this work, and therefore specific obstacles are explained in the following chapters.

\subsection{1 "Glass Radical"}

After changing the laser type, an unknown EPR signal was observed during the measurements of the copolymerization of styrene and MMA $\left(f_{\text {Sty }}=0.498\right.$ and $\left.f_{\mathrm{MMA}}=0.502\right)$ (Figure 3.6). To clarify the origin of this signal, it was tested whether the signal is caused by the photoinitiator or by the monomer. Finally, it turned out that this signal was caused by the sample tube. Therefore, this unknown signal was named "glass radical" which was stable over days. It turned out that similar problems were observed by another working group in IR spectra after the excimer laser was exchanged by a Nd:YAG laser. ${ }^{[97]}$ Therefore, it was recommended to install a telescope for beam homogenization in-between the laser and spectrometer. ${ }^{[97]}$ Additionally, the laser intensity was reduced. Afterwards, the signal of the "glass radical" was not observed anymore. 


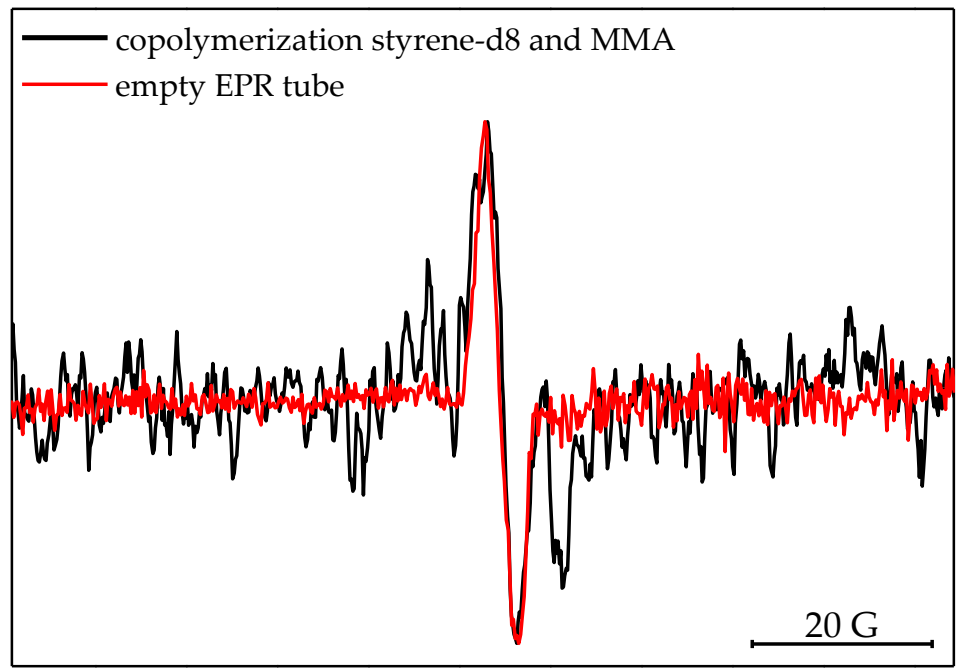

Figure 3.6: Spectrum recorded during the copolymerization of styrene- $\mathrm{d} 8$ and MMA $\left(f_{\text {Sty }}=0.498\right.$ and $\left.f_{\mathrm{MMA}}=0.502\right)$ in bulk at $333 \mathrm{~K}$ is shown in black. The spectrum for an empty EPR tube. Both spectra were recorded under pseudo-stationary conditions with a pulse repetition rate of $30 \mathrm{~Hz}$ and are normalized.

\subsubsection{EPR Signal of Oxygen}

Comparing the two spectra recorded during a radical homopolymerization of PnMA (Figure 3.7), the red spectrum represents the characteristically spectrum for methacrylates which will be explained in Chapter 4.1 whereas an additional signal is observed in the black spectrum. This additional signal can be assigned to oxygen. Oxygen is an inhibitor, and thus it directly influences the termination kinetics. Consequently, it has to be avoided for SP experiments. Especially for the small tubes, the present of oxygen was an issue since the caps could not be fixed tightly to the tubes. The presence of oxygen alters the concentration-time profile and even makes the measurements impossible. Thus, before the SP experiments, a spectrum must be recorded to ensure the absence of oxygen: the author highly recommends measuring only 2 or 3 samples in a row and not perform any SP experiments if an oxygen signal is observed. 


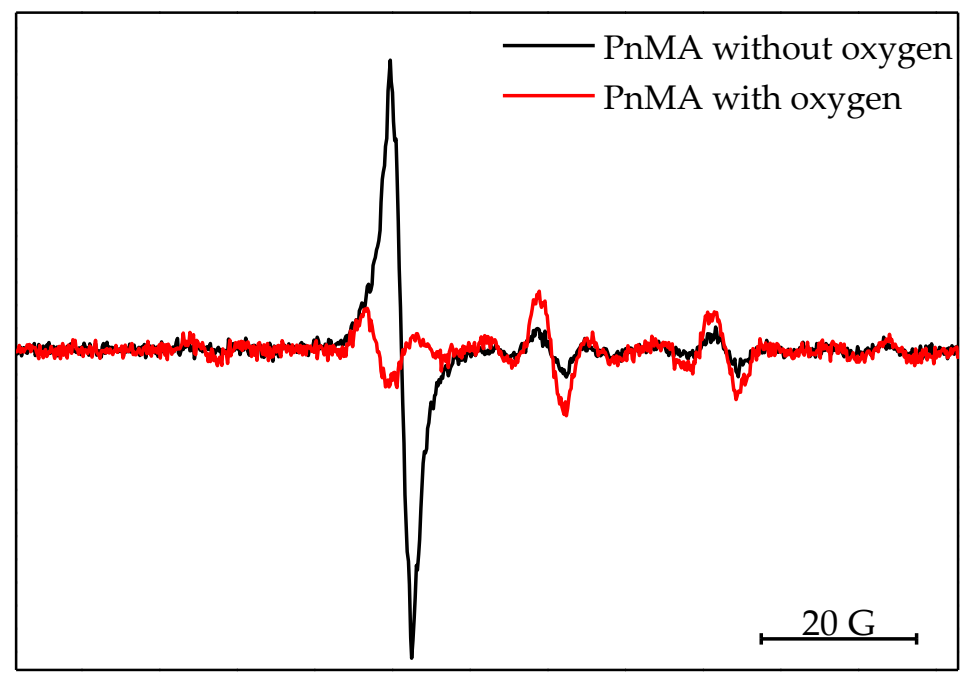

Figure 3.7: Both spectra were recorded during a PnMA homopolymerization at $233 \mathrm{~K}$ in bulk under pseudo stationary conditions (p.r.r. $30 \mathrm{~Hz}$ ). The black one shows an oxygen signal whereas no oxygen is observed in the red ones.

\subsubsection{EPR Signal of the Heating Element}

It was known that the heating element in the cavity also induces an EPR signal. This signal is reproducible for the given conditions. Especially, for the recorded spectra during the homopolymerization of styrene-d8 and the copolymerization of styrene-d8 and MMA, the signal of the heating element disturbs the measurement because of the low signal intensity of the sample radicals. To solve this issue, the spectra of copolymerization and styrene-d8 were averaged from up to 60 spectra (shown in black in Figure 3.8) and the backgrounds were averaged over 10 measurement (shown in red in Figure 3.8). In this way, the signal from the hating elements can be successful subtracted from the sample spectrum. 


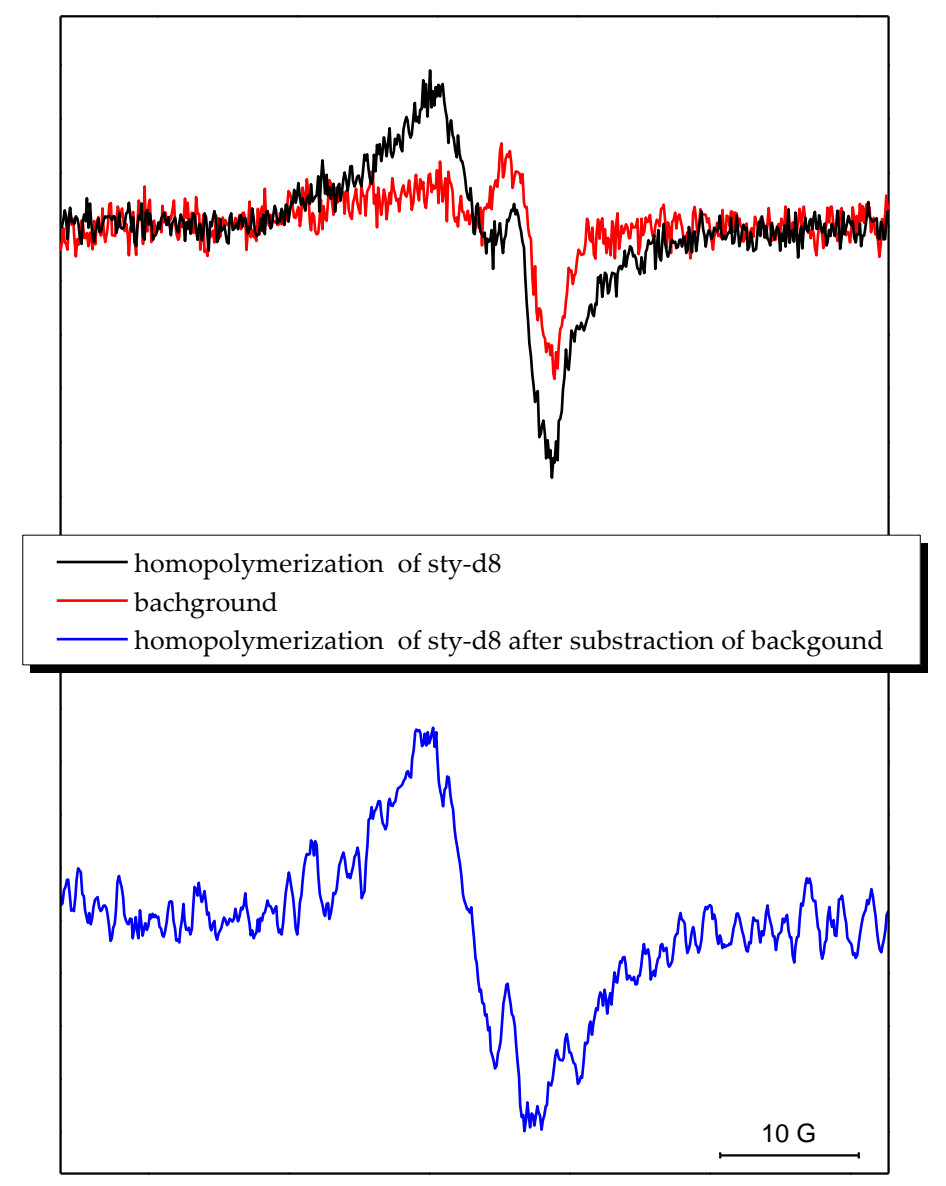

Figure 3.8: Spectrum recorded during a radical homopolymerization of styrene-d8 in bulk at $353 \mathrm{~K}$ (black). The background was measured before the irradiation (red). After subtracting the background, the spectrum of the homopolymerization of styrene- $\mathrm{d} 8$ can be obtained without the signal of the heating element. 


\section{TERMINATION KINETICS OF RADICAL POLYMERIZATION OF METHACRYLATES}

Methacrylates are one of the most important monomer families used for polymerizations. The most famous one is poly(methyl methacrylate) (PMMA) which is known as Plexiglass ${ }^{\circledR[}{ }^{[3]}$ Furthermore, several methacrylates are widely used for copolymerizations as can be seen in Chapter 5. Thus, the knowledge of the kinetics of this monomer family is essential for planning radical polymerizations, and moreover for further investigations into the kinetics of copolymerizations. The kinetics of some monomers within this monomer family is well investigated and a family behavior for the propagation kinetics could be found. ${ }^{[98]}$ Nevertheless, the detailed kinetics of some methacrylates are still unknown and some questions about the crossover chain length were left open in previous studies, too. ${ }^{[5]}$

One interesting monomer within the methacrylate family is $n$-pentyl methacrylate (PnMA). Due to its glass transition temperature slightly below room temperature, poly-PnMA is an interesting matrix polymer for testing the mechanical properties of polymer-filler compounds. ${ }^{[5]}$ However, nothing was known about the kinetics of PnMA so far. Therefore, the propagation kinetics of PnMA was investigated by $A$. Nitschke and coworkers and the results are discussed in the relating publication. ${ }^{[99]}$ Moreover, the chain-length dependent termination kinetics of PnMA was studied in detail within this work using the powerful SP-PLP-EPR method. The obtained results are discussed in this chapter.

i The results of PnMA in this chapter were published in A. Nitschke, L. Riemann, L. Kollenbach, V. Braun, M. Buback, P.Vana, Macromolecular Chemistry and Physics 2019, 221(1), 1900345 and are reproduced with permission. ${ }^{\text {[99] }}$ 
In a previous study of the termination kinetics of methacrylates, a significant temperature dependency of $i_{\mathrm{c}}$ was observed for 2-ethyl hexyl methacrylate (2-EHMA) and dodecyl methacrylate (DMA). ${ }^{[55]}$ This was surprising because such a temperature dependency of $i_{\mathrm{c}}$ was not observed for other methacrylates such as butyl methacrylate (BMA $)^{[31]}$ and acrylates, ${ }^{[42]}$ respectively. Nonetheless, the focus of the previous study was not on this temperature dependency. Therefore, more detailed investigations into the temperature dependency of $i_{\mathrm{c}}$ for 2-EHMA and DMA in bulk were performed within this work and the results are discussed in this chapter.

Beside this interest into the termination kinetics of this monomer family, several parts of the experimental setup had to be replaced or repaired in comparison to previous works. Especially, the excimer laser was exchanged by an Nd:YAG laser which led to problems such as formation of a glass radical (Chapter 3.3.1). Hence, it was essential to verify that the new results are reliable. Because of the investigations into the termination kinetics of numerous methacrylates, this monomer family is an excellent candidate to validate the changes within the experimental setup.

\subsection{EPR Spectrum of Pentyl Methacrylate}

The experimental EPR spectrum recorded during a homopolymerization of PnMA in bulk at $233 \mathrm{~K}$ (black line in Figure 4.2) shows the characteristic thirteen signals for methacrylates. ${ }^{[31,55,100]}$ Due to the methyl group, the rotation around the $\mathrm{C}_{\alpha}-\mathrm{C}_{\beta}$-bond is hindered, and thus two conformers of methacrylates exist (Figure 4.1). As can be seen, the dihedral angles $\Theta$ between p-orbital containing the radical and the hydrogens of the methylene group differ. $\Theta$ can be correlated to the hyperfine coupling constant $a_{\mathrm{hf}}$ by the Heller-McConnell equation (Equation 4.1). ${ }^{[101-103]}$ Hence, the hydrogen atoms of the methylene group have inequivalent $a_{\mathrm{hf}}$.

$$
a_{\mathrm{hf}}=A \cdot \cos ^{2}(\Theta)
$$




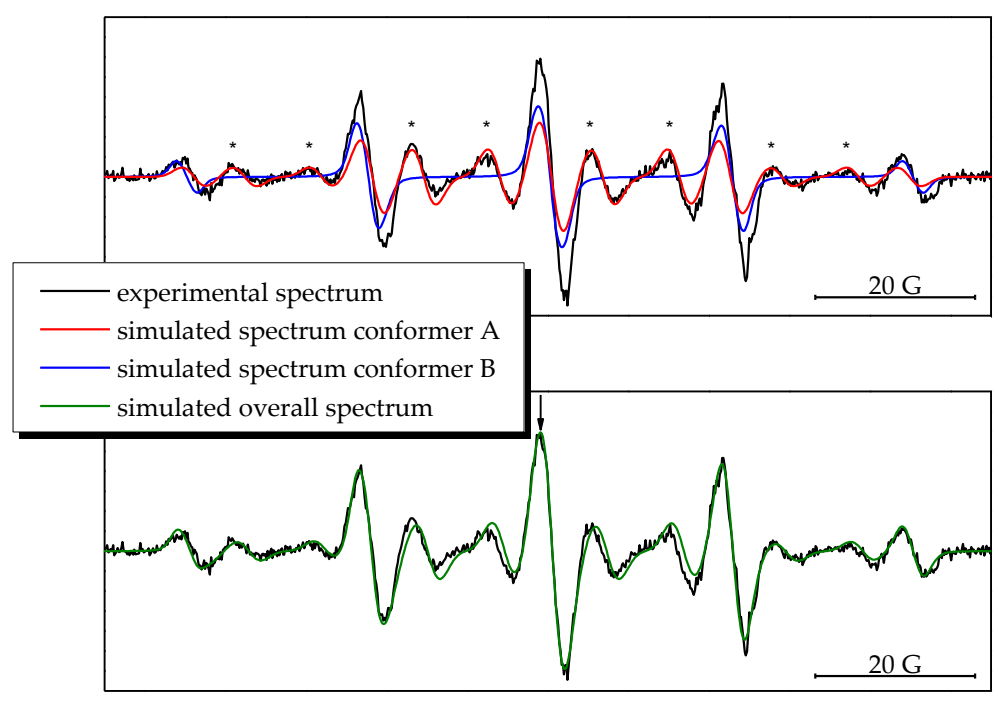

Figure 4.2: EPR spectrum (black) recorded during a PnMA polymerization at $233 \mathrm{~K}$ with DCP $\left(9 \cdot 10^{-2} \mathrm{~mol} \cdot \mathrm{L}^{-1}\right)$ as the photoinitiator under laser pulsing with a repetition rate of $30 \mathrm{~Hz}$. Red and blue are the simulated spectra for both conformers with the hyperfine coupling constants given in Table 4.1. Superposition of the two conformers spectra lead to the overall spectrum in green. The arrow indicates the magnetic field position used within the SP-PLP-EPR experiments.

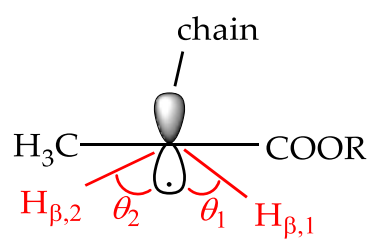

conformer A

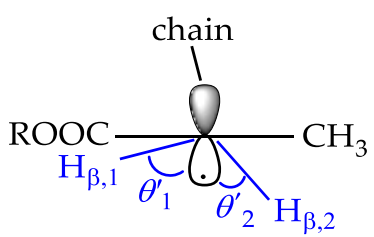

conformer B

Figure 4.1: Newman projection of the two conformers of methacrylates.

$A$ is a proportionality constant. The simulated spectra for both conformers of PnMA are shown in red and blue in Figure 4.2. From simulations, the hyperfine coupling constants for the three equivalent hydrogen atoms of the methyl group and for the two inequivalent hydrogen atoms of the methylene group were obtained and are given in Table 4.1. 
Table 4.1: $\quad$ Hyperfine coupling constants $a_{\mathrm{hf}}$ obtained from fitting the spectrum shown in Figure 4.2 for the different conformers of PnMA at $233 \mathrm{~K}$.

\begin{tabular}{ccc}
\hline & Conformer A & Conformer B \\
\hline \hline $\begin{array}{c}\text { Amount and position } \\
\text { of hydrogen }\end{array}$ & $a_{\mathrm{hf}} / \mathrm{G}$ & $a_{\mathrm{hf}} / \mathrm{G}$ \\
$\mathbf{1} \mathbf{H}_{\beta, 1}$ & 15.8 & 23.5 \\
$\mathbf{1 ~ H}_{\beta, 2}$ & 6.3 & 0.2 \\
$\mathbf{3} \mathbf{H}_{\text {methyl }}$ & & 22.3 \\
\hline
\end{tabular}

Moreover, superimposing of both conformer spectra lead to the overall spectrum shown in green in Figure 4.2 which perfectly agrees with the experimental spectrum of PnMA. Furthermore, the ratio of the two conformers can be determined by simulation and is around 1:1 at $233 \mathrm{~K}$. As can be seen in Figure 4.2, the small inner lines of the spectrum marked with asterisks only belongs to conformer A shown in red. With increasing temperature, these inner lines increase because of the improved rotation around the $\mathrm{C}_{\alpha}-\mathrm{C}_{\beta}$-bond as will be shown for MMA at $333 \mathrm{~K}$ in Chapter 5.1. However, the ratio of conformers is unimportant for determining the termination kinetics. The understanding of the spectra is important to be sure that no other radicals - such as oxygen for example (Chapter 3.3.2)-are formed which may influence the termination kinetics.

\subsection{Chain-Length Dependent Termination of Pentyl Methacrylate}

For determining the chain-length dependent termination of PnMA, single pulse experiments were performed at the static field position indicated with an arrow in Figure 4.2. The signal-to-noise ratio was improved by co-adding up to 10 individual concentration-time profiles. After each single pulse experiment the monomer-to-polymer conversion was checked gravimetrically and was below $7 \%$. Moreover, three samples were measured for each temperature and the determined composite parameters were averaged. The so-obtained concentration-time profiles at $233 \mathrm{~K}$ and $313 \mathrm{~K}$ are shown in Figure 4.3. As expected, the 


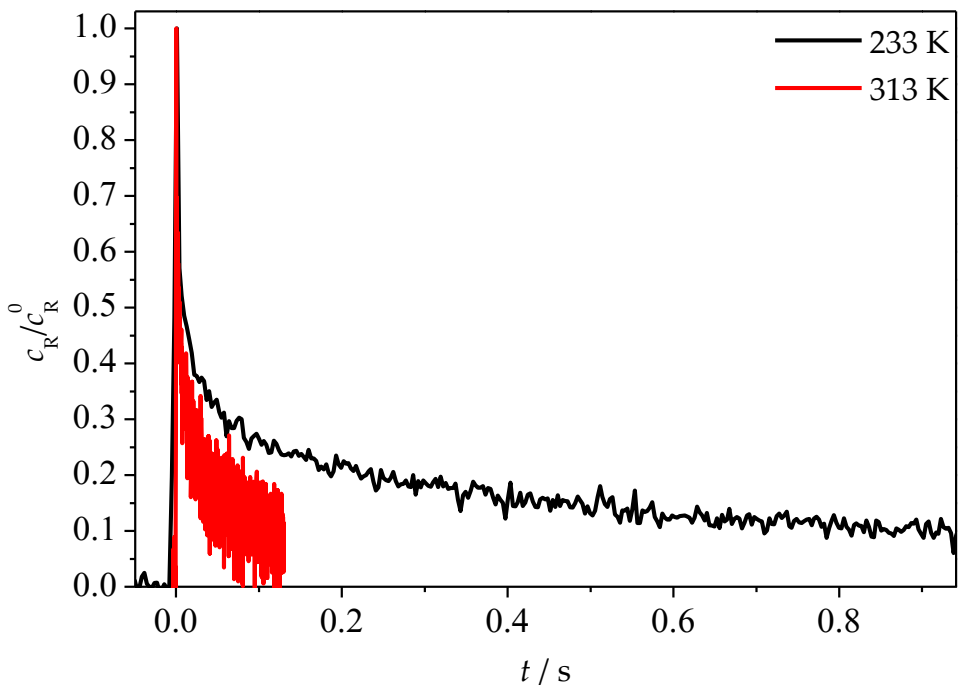

Figure 4.3: Normalized concentration-time profiles for $233 \mathrm{~K}$ (black) and $313 \mathrm{~K}$ (red) for PnMA in bulk with DCP used as photoinitiator $\left(9 \cdot 10^{-2} \mathrm{~mol} \cdot \mathrm{L}^{-1}\right)$. For each concentration-time profile, up to 10 traces were co-added for improving the signal-to-noise ratio. The static field position is indicated with an arrow in Figure 4.2.

concentration decay at $313 \mathrm{~K}$ is much faster than for $233 \mathrm{~K}$ caused by the faster termination at higher temperature. However, the higher signal-tonoise ratio at $233 \mathrm{~K}$ is caused by the Boltzmann distribution and the higher time resolution.

The obtained data from SP experiments were evaluated as recommended (see Chapter 2.6). ${ }^{[104]}$ Thus, the composite parameters $\alpha_{1}$ and $i_{\mathrm{c}}$ were obtained by a double logarithmic plot of the experimental data. In Figure 4.4, the double logarithmic plot is shown for PnMA polymerization in bulk at $233 \mathrm{~K}$. The short chain region (red line) and the long chain region (blue line) are linearly fitted. According to Equation 4.2, the slope of the linear fit of the long chain region is equal to $\left(1-\alpha_{1}\right)$.

$$
\log \left(\frac{c_{\mathrm{R}}^{0}}{c_{\mathrm{R}}(t)}-1\right)=\log \left(\frac{2 \cdot k_{\mathrm{t}}^{0} \cdot c_{\mathrm{R}}^{0} \cdot t_{\mathrm{p}}{ }^{\alpha_{1}}}{1-\alpha_{1}}\right)+\left(1-\alpha_{1}\right) \cdot \log (t) \quad i \gg i_{\mathrm{c}}
$$




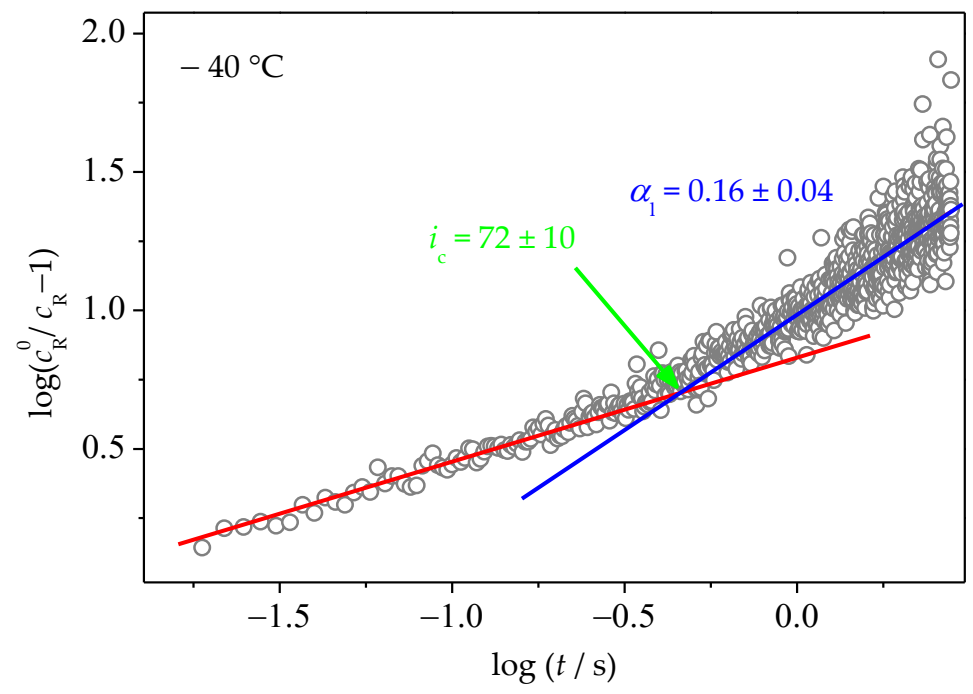

Figure 4.4: Double-log plot of data obtained by SP-PLP-EPR measurements of PnMA bulk polymerization at $233 \mathrm{~K}$ and low degrees of monomer conversion. From the slope of the two straight-lines, the power-law exponents for short-chain and long-chain radicals were determined, respectively. The intersection of the two straight lines yields the time $t_{\mathrm{c}}$

From the intersection of the two linear fits, the crossover time $t_{\mathrm{c}}$ can be determined, and thus $i_{\mathrm{c}}$ can be calculated by Equation 2.44. It should be noted that for the data treatment so far, no calibration is required because the ratio of radical concentration $\frac{c_{R}^{0}}{c_{R}(t)}$ is equal to the intensity ratio $\frac{I_{0}}{I(t)}$ where $I_{0}$ is the initial intensity and $I(t)$ the intensity at time $t$. For Equation 2.44, the monomer concentration $c_{\mathrm{M}}$ and the propagation rate coefficient $k_{\mathrm{p}}$ are required. The $c_{\mathrm{M}}$ is calculated by the molar mass and the temperature dependent density which was determined by A. Nitschke and coworkers who also determined $k_{\mathrm{p}} \cdot{ }^{\left[{ }^{[9]}\right.}$ Moreover, $\alpha_{\mathrm{s}}$ might be obtained by the slope of the linear fit of the short chain region. As mentioned in Chapter 2.6, however, this would lead to systematic errors because the initiator fragment is not considered in Equation 2.44. ${ }^{[91]}$ Consequently, the short chain region is evaluated by a least-squares fit of the experimental data (see Figure 4.5). Hence, $\alpha_{\mathrm{s}}$ is directly obtained by the least-squares 


\subsection{Chain-Length Dependent Termination of Pentyl Methacrylate}

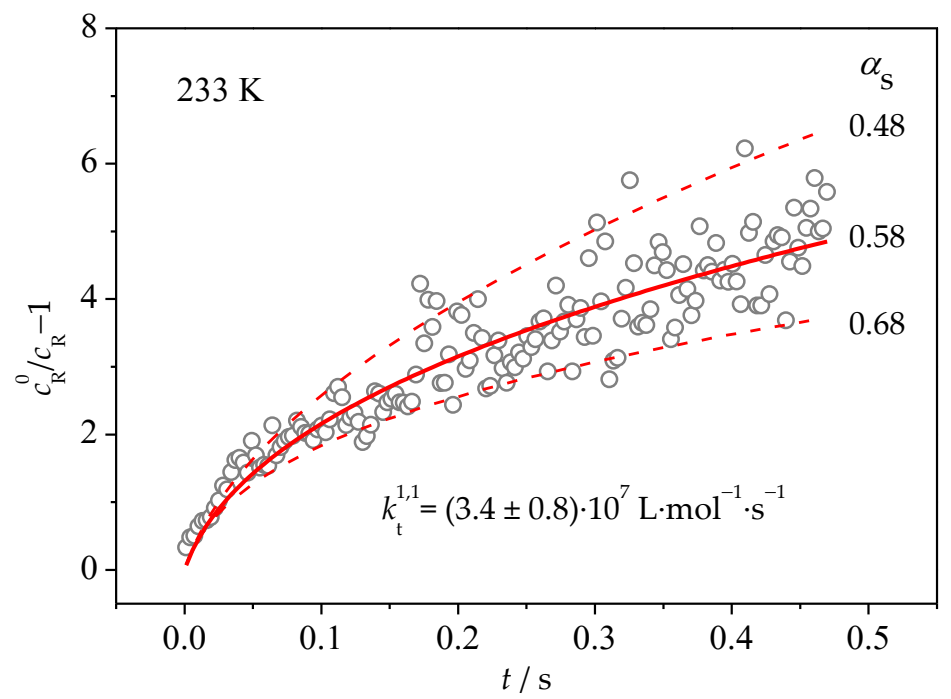

Figure 4.5: Least-squares fit of the experimental data for PnMA polymerization in bulk at $233 \mathrm{~K}$ to determine the power-law exponent $\alpha_{\mathrm{s}}$ and the termination rate coefficient of two monomeric radicals $k_{\mathrm{t}}^{1,1}$.

fit based on Equation 2.47. Furthermore, the product $k_{\mathrm{t}}^{1,1} \cdot c_{\mathrm{R}}^{0}$ is obtained. The absolute initial radical concentration $c_{\mathrm{R}}^{0}$ is calculated by the calibration procedure (see Chapter 3.2.5), and thus $k_{\mathrm{t}}^{1,1}$ is determined.

The so-obtained $\alpha_{\mathrm{s}}$ (red squares) and $\alpha_{1}$ (blue squares) values are shown in Figure 4.6 where the open symbols refer to the individual samples and the full symbols represent the associated arithmetic mean values. As can be seen, both power-law exponents are insensitive to temperature, and thus the overall arithmetic mean values $\alpha_{\mathrm{s}}=0.56$ and $\alpha_{1}=0.16$ are given as full lines. In comparison to other alkyl methacrylates and monomers (Table 4.2), $\alpha_{\mathrm{s}}$ and $\alpha_{1}$ for PnMA agrees perfectly. Additionally, for the other monomers no temperature dependence of $\alpha_{\mathrm{s}}$ and $\alpha_{1}$ was observed. 


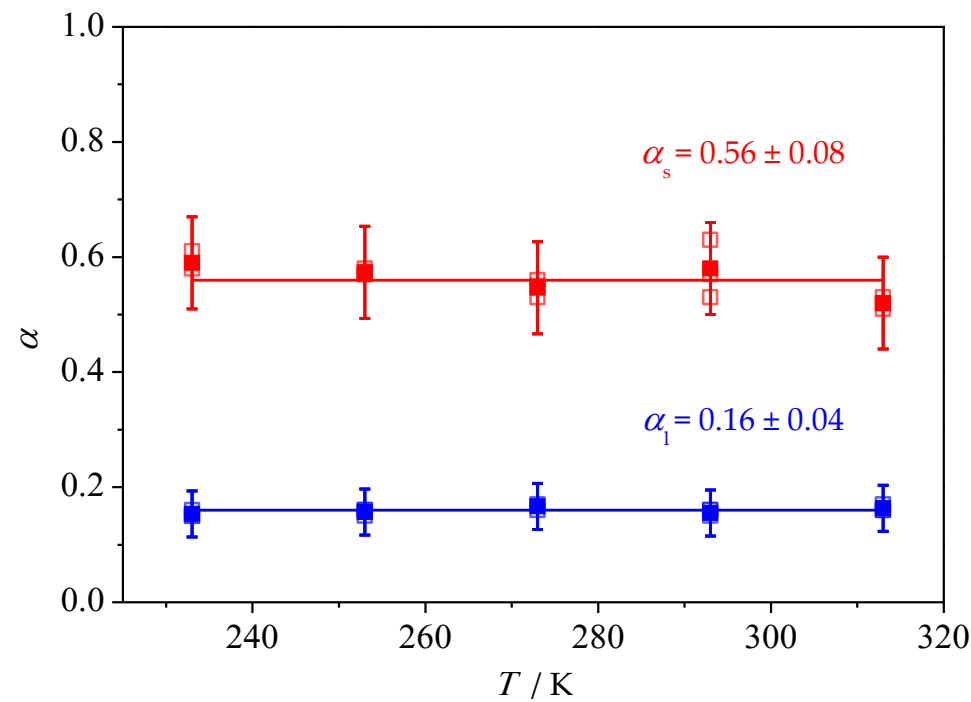

Figure 4.6: Power-law exponent $\alpha_{\mathrm{s}}$ (red squares) for short-chain radicals and $\alpha_{1}$ (blue squares) for long-chain radicals in bulk PnMA polymerization at different temperatures $T$. The open symbols refer to individual experiments whereas the full symbols represent the associated arithmetic mean value. The lines indicate that both power-law exponents are not sensitive toward temperature.

Table 4.2: $\quad$ Power-law exponents $\alpha_{\mathrm{s}}$ for short chain region and $\alpha_{1}$ for the long chain region for alkyl methacrylates and other monomers. MMA: methyl methacrylate, BMA: butyl methacrylate, 2-EHMA: 2-ethylhexyl methacrylate, DMA: dodecyl methacrylate.

\begin{tabular}{cccc}
\hline monomer & $\alpha_{\mathrm{s}}$ & $\alpha_{1}$ & ref. \\
\hline \hline MMA & $0.63 \pm 0.15$ & $0.16-0.17$ & {$[30,105]$} \\
BMA & $0.65 \pm 0.15$ & $0.20 \pm 0.05$ & {$[31]$} \\
tert-BMA & $0.56 \pm 0.15$ & $0.20 \pm 0.05$ & {$[31]$} \\
PnMA & $0.56 \pm 0.08$ & $0.16 \pm 0.04$ & this work \\
2-EHMA & $0.61 \pm 0.09$ & $0.19 \pm 0.05$ & {$[55]$} \\
DMA & $0.65 \pm 0.08$ & $0.17 \pm 0.04$ & {$[55]$} \\
\hline \hline styrene & $0.51 \pm 0.05$ & $0.16 \pm 0.05$ & {$[34]$} \\
vinyl acetate & $0.57 \pm 0.05$ & $0.16 \pm 0.07$ & {$[94]$} \\
vinyl pivalate & $0.67 \pm 0.15$ & $0.16 \pm 0.07$ & {$[94]$} \\
\hline
\end{tabular}




\subsection{Chain-Length Dependent Termination of Pentyl Methacrylate}

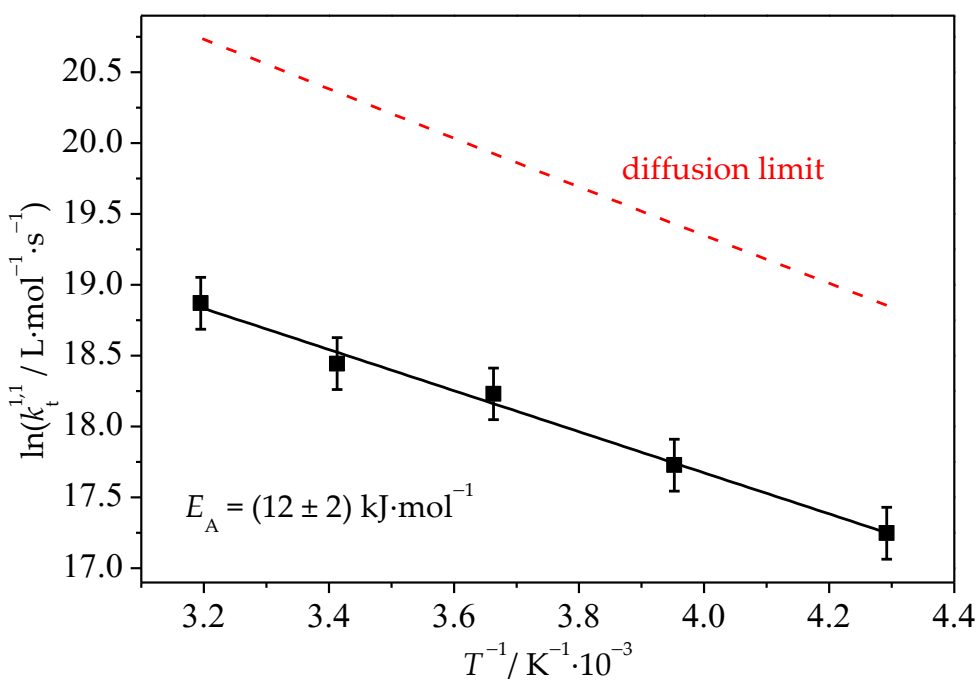

Figure 4.7: Arrhenius plot of the measured $k_{t}^{1,1}$ for PnMA bulk polymerization. The dashed red line represents $k_{t}^{1,1}$ as estimated from diffusion limit (Equation 2.25).

Furthermore, depending on the structure of the macroradical (coil or rodlike), $\quad \alpha_{\mathrm{s}}=0.5-1.0$ are theoretically predicted. ${ }^{[29,106,107]}$ For macroradicals with the radical functionality at the chain-end, $\alpha_{1}=0.16$ is theoretically predicted. ${ }^{[29,108]}$ Hence, the obtained $\alpha_{\mathrm{s}}$ and $\alpha_{1}$ fully assent to the theoretical predicted values as well as to the experimental values for other methacrylates (Table 4.2). The results for $i_{\mathrm{c}}$ will be discussed in Chapter 4.3 in detail.

The resulting $k_{t}^{1,1}$ are given as an Arrhenius plot in Figure 4.7. By linear fitting of the determined $k_{\mathrm{t}}^{1,1}$, an Arrhenius equation of $k_{\mathrm{t}}^{1,1}$ is obtained as follows:

$$
\ln \left(k_{\mathrm{t}}^{1,1} / \mathrm{L} \cdot \mathrm{mol}^{-1} \cdot \mathrm{s}^{-1}\right)=23.47-1449 \cdot(\mathrm{T} / \mathrm{K})^{-1} .
$$

Additionally, the diffusion limit is shown in Figure 4.7 as red dashed line and is calculated by Equation 2.25. The diffusion limit indicates the maximal theoretical limit for $k_{\mathrm{t}}^{1,1}$ at a given viscosity $\eta$ and temperature $T$. As can be seen, the determined $k_{t}^{1,1}$ are lower than the diffusion limit 
Table 4.3: The activation energy $E_{\mathrm{A}}\left(k_{\mathrm{t}}^{1,1}\right)$ and pre-exponential factor $A\left(k_{\mathrm{t}}^{1,1}\right)$ for the termination rate coefficient of two monomeric radicals $k_{\mathrm{t}}^{1,1}$ for different n-alkyl methacrylates and monomers.

\begin{tabular}{cccc}
\hline monomer & $E_{\mathbf{A}}\left(\boldsymbol{k}_{\mathbf{t}}^{\mathbf{1 , 1}}\right) / \mathbf{~} \mathbf{J} \cdot \mathbf{m o l}^{-\mathbf{1}}$ & $\begin{array}{c}A\left(\boldsymbol{k}_{\mathbf{t}}^{\mathbf{1 , 1}}\right) / \mathbf{1 0} \\
\mathbf{L} \cdot \mathbf{m o l}^{\mathbf{1 0}} \cdot \mathbf{s}^{-\mathbf{1 0}}\end{array}$ & ref. \\
\hline \hline MMA & $9 \pm 2$ & 2.3 & {$[30,105]$} \\
BMA & $10 \pm 2$ & $1.1^{*}$ & {$[31]$} \\
tert-BMA & $12 \pm 2$ & $1.8^{*}$ & {$[31]$} \\
PnMA & $12 \pm 2$ & 1.6 & this work \\
2-EHMA & $17 \pm 2$ & 2.7 & {$[55]$} \\
DMA & $20 \pm 2$ & 7.9 & {$[55]$} \\
\hline \hline styrene & $9 \pm 1$ & 1.9 & {$[34]$} \\
vinyl acetate & $9 \pm 2$ & 3.2 & {$[94]$} \\
vinyl pivalate & $12 \pm 2$ & 2.6 & {$[94]$} \\
\hline
\end{tabular}

* calculated from the given $E_{\mathrm{A}}$ and $k_{\mathrm{t}}^{1,1}$ in the literature.

which demonstrates that the obtained $k_{\mathrm{t}}^{1,1}$ are reliable. In comparison with other $n$-alkyl methacrylates (Table 4.3), the values for PnMA closely fit into the tendency that activation energy $E_{\mathrm{A}}\left(k_{\mathrm{t}}^{1,1}\right)$ and the preexponential factor $A\left(k_{\mathrm{t}}^{1,1}\right)$ increases with increasing size of ester side chain. A similar tendency was also found for vinyl esters (Table 4.3). This can be explained by increasing shielding effects with an increasing size the of ester side chain.

As described in Chapter 2.3.3, if the translational diffusion is as dominant as it is the case for short chains, and thus for $k_{t}^{1,1}$, the Smoluchowski equation (Equation 2.23) ${ }^{[71]}$ and the Stokes-Einstein equation (Equation 2.7) ${ }^{[57]}$ can be used for $k_{t}^{1,1}$. Moreover, with these two equations, a direct relation between $k_{\mathrm{t}}^{1,1} \cdot \eta$ and ratio of $R_{\mathrm{c}}$ and $r_{1}$ is obtained which provides a direct comparison of the influence of the monomeric structure on $k_{\mathrm{t}}^{1,1}$ (Equation 4.4). 
Table 4.4 Termination rate coefficient of two monomeric radicals $k_{\mathrm{t}}^{1,1}$, viscosity $\eta$, and the product of both quantities for several alkyl methacrylates and monomers at $80^{\circ} \mathrm{C}$.

\begin{tabular}{|c|c|c|c|c|}
\hline Monomer & $\begin{array}{c}k_{\mathrm{t}}^{1,1}\left(80^{\circ} \mathrm{C}\right) / \\
10^{9} \mathrm{~L}^{\prime} \cdot \mathrm{mol}^{-1} \cdot \mathrm{s}^{-1}\end{array}$ & $\begin{array}{l}\eta\left(80^{\circ} \mathrm{C}\right) \\
/ \mathrm{mPa} \cdot \mathrm{s}\end{array}$ & $\begin{array}{c}k_{\mathrm{t}}^{1,1} \eta\left(80^{\circ} \mathrm{C}\right) / \\
10^{8} \\
\mathrm{~L} \cdot \mathrm{mPa} \cdot \mathrm{mol}^{-1}\end{array}$ & ref. \\
\hline MMA & $(1.1 \pm 0.3)$ & 0.34 & 3.7 & {$[30,105]$} \\
\hline BMA & $(0.36 \pm 0.07)$ & 0.43 & 1.5 & [31] \\
\hline tert-BMA & $(0.3 \pm 0.1)$ & 0.45 & 1.3 & [31] \\
\hline PnMA & $(0.26 \pm 0.08)$ & 0.50 & 1.3 & $\begin{array}{l}\text { this } \\
\text { work }\end{array}$ \\
\hline 2-EHMA & $(0.095 \pm 0.015)$ & 0.73 & 0.7 & [55] \\
\hline DMA & $(0.088 \pm 0.015)$ & 1.29 & 1.1 & [55] \\
\hline styrene & $(0.83 \pm 0.05)$ & 0.39 & 3.2 & [34] \\
\hline vinyl acetate & $(1.5 \pm 0.3)$ & 0.24 & 3.6 & [94] \\
\hline vinyl pivalate & $(0.41 \pm 0.05)$ & 0.33 & 1.4 & [94] \\
\hline
\end{tabular}

Since the activation energy of the fluidity $E_{\mathrm{A}}\left(\eta^{-1}\right)=12 \mathrm{~kJ} \cdot \mathrm{mol}^{-1}$ for PnMA (see Appendix II) is similar to $E_{\mathrm{A}}\left(k_{\mathrm{t}}^{1,1}\right)$, the product $k_{\mathrm{t}}^{1,1} \eta$ is constant for different temperatures. This was also found for other methacrylates and monomers. In comparison to those (Table 4.4), a tendency for $k_{\mathrm{t}}^{1,1} \cdot \eta$ is observed. The highest value for $k_{\mathrm{t}}^{1,1} \cdot \eta$ is determined for MMA which is close to the ones for vinyl acetate and styrene. Moreover, with increasing size of ester side chain, $k_{\mathrm{t}}^{1,1} \cdot \eta$ decreases. The increasing size of ester side chain leads to a higher $r_{1}$, and hence $k_{\mathrm{t}}^{1,1} \cdot \eta$ becomes smaller (Equation 4.4). Thus, the influence of the structure on $k_{\mathrm{t}}^{1,1}$ becomes obvious. Due to the precise knowledge of the propagation and termination kinetics obtained for PnMA, kinetic modelling is possible to obtain a well-defined polymeric product. 


\subsection{Crossover Chain Length for Various Methacrylates}

The determined crossover chain length $i_{\mathrm{c}}$ for PnMA at several temperatures is depicted in Figure 4.8 where the open symbols refer to the different samples and the full symbols represent the associated arithmetic mean. From $273 \mathrm{~K}$ to $293 \mathrm{~K}$, a small decrease of $i_{\mathrm{c}}$ is observed. However, this effect is within the experimental accuracy, and thus it is not a clear temperature dependency. The arithmetic mean value of $i_{\mathrm{c}}$ for PnMA over all temperatures is $i_{\mathrm{c}}=67 \pm 10$. Within the methacrylate family (Table 4.5), no clear tendency for $i_{\mathrm{c}}$ can be noted. Compared to the other methacrylates, $i_{\mathrm{c}}$ for PnMA is close to the value for tert-BMA and inbetween the value for MMA and BMA. In comparison with other monomer families, $i_{\mathrm{c}}$ for PnMA is close to the value for butyl acrylate (BA, $i_{\mathrm{c}}=70 \pm 15$ ). Furthermore, $i_{\mathrm{c}}$ is lower for methyl acrylate (MA), styrene and vinyl acetate than for PnMA whereas the $i_{\mathrm{c}}$ for vinyl pivalate is

Table 4.5: Crossover chain length $i_{\mathrm{c}}$ for different monomers. The values were determined by different methods as noted. Some of the $i_{\mathrm{c}}$ were obtained over a wide temperature range and the presented values are averaged over all temperature.

\begin{tabular}{|c|c|c|c|c|c|}
\hline monomer & $i_{\mathrm{c}}$ & solvent & $T / \mathrm{K}$ & method & ref. \\
\hline MMA & 100 & bulk & 353 & RAFT-CLD-T & {$[30,105]$} \\
\hline BMA & $50 \pm 15$ & bulk & $243-333$ & SP-PLP-EPR & [31] \\
\hline tert-BMA & $70 \pm 15$ & bulk & $293-333$ & SP-PLP-EPR & [31] \\
\hline PnMA & $67 \pm 10$ & bulk & $233-313$ & SP-PLP-EPR & $\begin{array}{l}\text { this } \\
\text { work }\end{array}$ \\
\hline MA & $35 \pm 10$ & $\begin{array}{l}1.5 \mathrm{M} \text { in } \\
\text { toluene }\end{array}$ & 233 & SP-PLP-EPR & [42] \\
\hline BA & $65 \pm 20$ & $\begin{array}{l}1.5 \mathrm{M} \text { in } \\
\text { toluene }\end{array}$ & 233 & SP-PLP-EPR & [42] \\
\hline styrene & $30 \pm 10$ & bulk & $346-408$ & SP-PLP-EPR & [34] \\
\hline $\begin{array}{l}\text { vinyl } \\
\text { acetate }\end{array}$ & $20 \pm 10$ & bulk & 208-333 & SP-PLP-EPR & [94] \\
\hline $\begin{array}{c}\text { vinyl } \\
\text { pivalate }\end{array}$ & $110 \pm 30$ & bulk & $208-333$ & SP-PLP-EPR & [94] \\
\hline
\end{tabular}




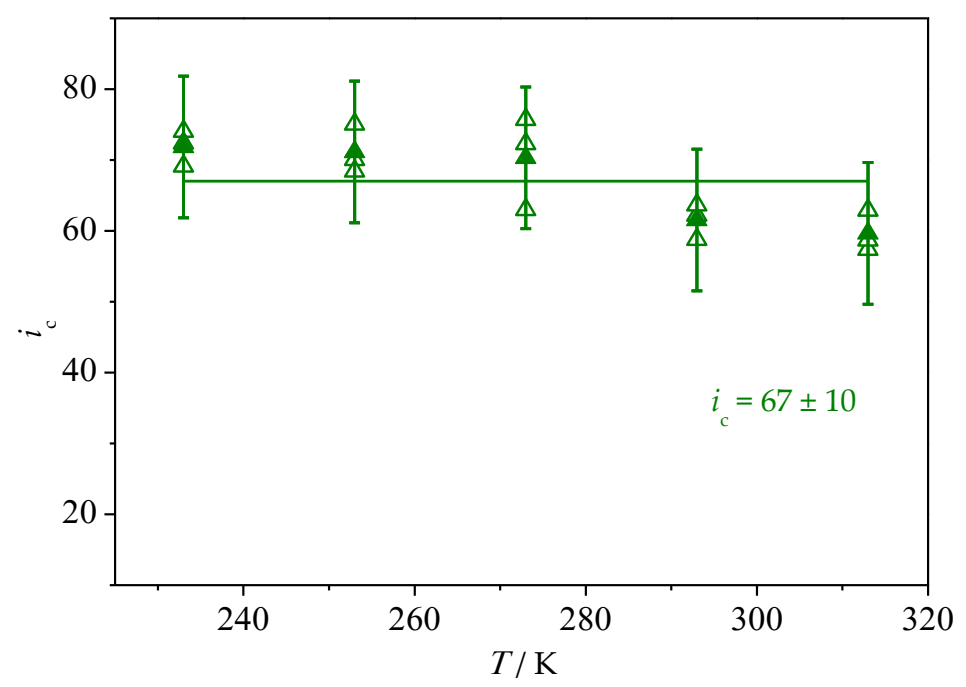

Figure 4.8: Crossover chain length $i_{\mathrm{c}}$ for bulk PnMA polymerization at several temperatures. The open symbols refer to individual experiments whereas the full symbols represent the associated arithmetic mean value. The full line represents the arithmetic mean value for the temperature range under investigation.

higher. Moreover, $i_{\mathrm{c}}$ for acrylates and vinyl esters with the smaller ester side chain (MA and vinyl acetate) are significantly lower than for the ones with longer ester side chains (BA and vinyl pivalate) while $i_{\mathrm{c}}$ for MMA is significantly higher than for PnMA and BMA. Hence, the influence of the monomer structure on $i_{\mathrm{c}}$ is not clear so far.

Hereinafter, the importance of the monomer structure for $i_{\mathrm{c}}$ will be discussed. In a study of Sörensen, a significant temperature dependency was observed for $i_{c}$ for DMA and 2-EHMA. ${ }^{[5]}$ The dependence of temperature on $i_{\mathrm{c}}$ has been observed in another study of Kattner, too. ${ }^{[56]}$ Nonetheless, some discrepancies between these studies were observed. Therefore, $i_{\mathrm{c}}$ was investigated for DMA and 2-EHMA in more detail again. In comparison to the previous study of 2 -EHMA, ${ }^{[55]} i_{\mathrm{c}}$ was determined for two or three different samples and the presented results are the associated arithmetic mean values. For DMA, $i_{\mathrm{c}}$ was determined 


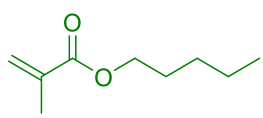

PnMA

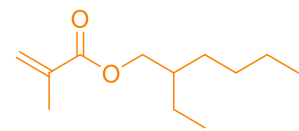

2-EHMA

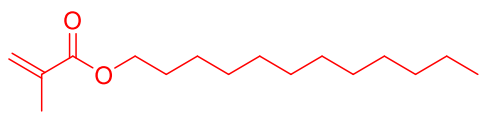

DMA

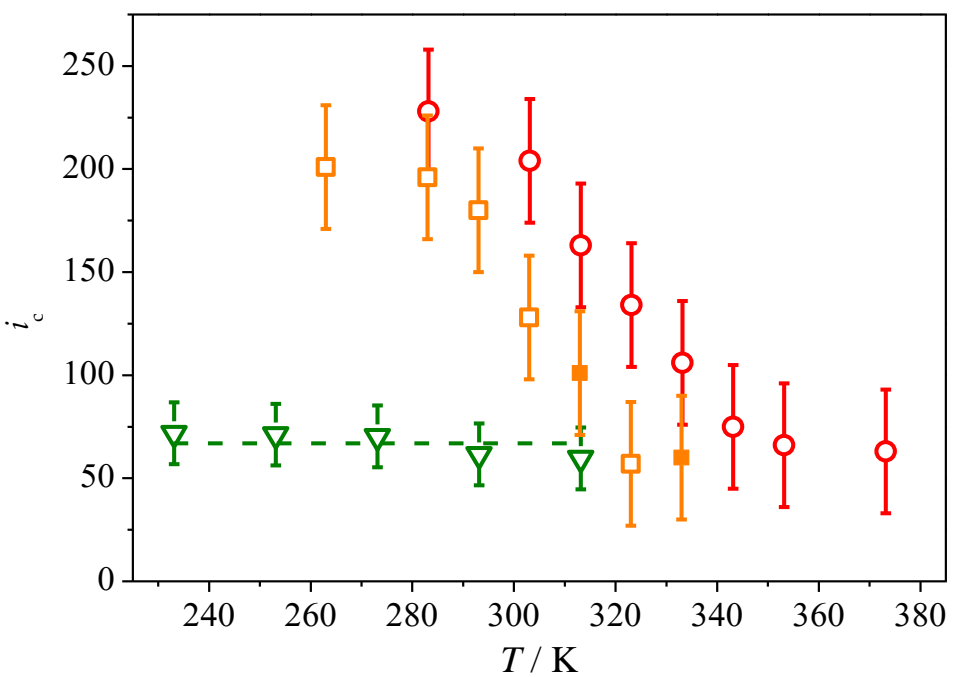

Figure 4.9: Crossover chain length $i_{\mathrm{c}}$ for bulk polymerizations of different methacrylates (PnMA green triangles, 2-EHMA orange squares, DMA red circles) for several temperatures. According to 2-EHMA, the full squares refer to experimental data which have to be measured again because of experimental problems. The structures for the different methacrylates are given above and the arrow indicates the significant influence of the ester side chain on $i_{\mathrm{c}}$. The green dashed line is the arithmetic mean value over all temperatures for PnMA $\left(i_{\mathrm{c}}=67 \pm 10\right)$.

in the previous study for even more samples $(\sim 5)$ but the temperature steps $(20 \mathrm{~K})$ were large. ${ }^{[55]}$ Within this work, in the region, where $i_{\mathrm{c}}$ significantly changes for DMA and 2-EHMA, $10 \mathrm{~K}$ steps were chosen. Therefore, $i_{\mathrm{c}}$ was determined as described for PnMA and the required density and propagation rate coefficient were taken from literature. ${ }^{[109]}$

The so-obtained results for $i_{\mathrm{c}}$ are shown in Figure 4.9. Compared to $i_{\mathrm{c}}$ for PnMA (green triangles), $i_{\mathrm{c}}$ for 2-EHMA (orange squares) and DMA (red circles) decreases significantly with increasing temperature. As can 
be seen (Figure 4.9), a sigmoidal behavior for $i_{\mathrm{c}}$ is detected for DMA and 2-EHMA. Instead, Sörensen observed an exponential decay of $i_{\mathrm{c}}$ with increasing temperature. This discrepancy might be explained by the fact that more data points were measured within this work, and temperature steps of $10 \mathrm{~K}$ were chosen within this work instead of $20 \mathrm{~K}$. Thus, the temperature dependency was investigated more precisely in this work. It has to be mentioned that the values marked as full squares relate to data which were obtained during experimental problems. These values have to be measured again to check whether they are reliable. Nevertheless, these values fit into the sigmoidal behavior, and thus they will be included in the further evaluation.

To quantify the sigmoidal behavior, the experimental data is fitted (Figure 4.10) with the subsequent equation:

$$
i_{\mathrm{c}}=i_{\mathrm{c}, \min }+\frac{\left(i_{\mathrm{c}, \max }-i_{\mathrm{c}, \min }\right)}{1+\exp \left(\frac{T-T_{\mathrm{i}}}{\mathrm{d} T}\right)},
$$

where $i_{c, \max }$ and $i_{c, \min }$ indicate the upper and the lower limit of crossover chain length, $T_{\mathrm{i}}$ is the inflection point and $\mathrm{d} T$ the slope of the middle section. The so-obtained parameters are given in Table 4.6. As can be seen, $i_{c, \text { min }}$ for 2-EHMA and DMA which are achieved at higher temperatures differ slightly from each other and are close to the ones reported value for $\mathrm{BMA}^{[31]}\left(i_{\mathrm{c}}=50 \pm 15\right)$ and $\operatorname{PnMA}\left(i_{\mathrm{c}}=67 \pm 10\right)$. Furthermore, the values of $i_{c \text {,max }}$, which is the low temperature case, differ for both monomers. Additionally, these values are significantly higher than for other monomers such as vinyl pivalate $\left(i_{\mathrm{c}}=110 \pm 30\right)^{[94]}$ and MMA $\left(i_{\mathrm{c}}=100\right),{ }^{[110]}$ respectively. However, $i_{c, \max }$ for DMA is similar to the reported one in the previous study $\left(i_{\mathrm{c}}(\mathrm{DMA}, 273 \mathrm{~K})=220 \pm 30\right) .{ }^{[55]} \mathrm{In}$ contrast, $i_{c, \text { max }}$ is significantly lower to the ones reported by Sörensen ${ }^{[55]}$ $\left(i_{\mathrm{c}}(2\right.$-EHMA, $\left.233 \mathrm{~K})=285 \pm 40\right)$. This can be explained by the higher number of samples measured for 2-EHMA in this work (3 samples for each temperature) compared to Sörensen (1 sample for each temperature). Regarding the inflection point $T_{\mathrm{i}}$, the value for 2-EHMA is $14 \mathrm{~K}$ lower than for DMA. Hence, this inflection point is shifted to higher temperatures with increasing size of ester side chain. This indicates the effect of the ester side chain length of the methacrylate on $i_{\mathrm{c}}$. 


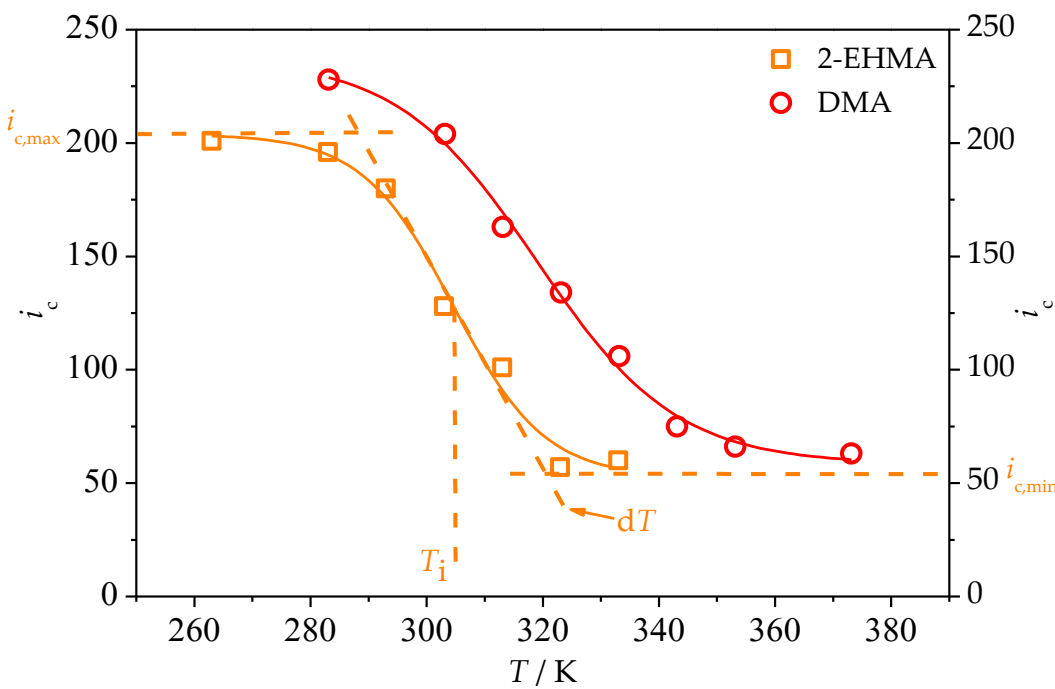

Figure 4.10: Sigmodal fitting temperature dependency of the crossover chain length for DMA and 2-EHMA. The solid lines show the sigmoidal fits (Equation 4.5). The obtained values from this fitting are shown exemplary for 2-EHMA as orange dashed lines where $i_{c, \max }$ and $i_{\mathrm{c}, \min }$ indicate the upper and the lower limit of crossover chain length, $T_{\mathrm{i}}$ is the inflection point and $\mathrm{d} T$ the slope of the middle section

Table 4.6: $\quad$ Parameter obtained by the sigmoidal fitting (Figure 4.10).

\begin{tabular}{ccccc}
\hline monomer & $\boldsymbol{i}_{\mathrm{c}, \min }$ & $\boldsymbol{i}_{\mathrm{c}, \max }$ & $\boldsymbol{T}_{\mathrm{i}} / \mathbf{K}$ & $\mathbf{d} \boldsymbol{T} / \mathbf{K}$ \\
\hline \hline 2-EHMA & $53 \pm 12$ & $204 \pm 10$ & $305 \pm 3$ & $7 \pm 3$ \\
DMA & $58 \pm 6$ & $238 \pm 9$ & $319 \pm 2$ & $12 \pm 2$ \\
\hline
\end{tabular}

To explain this temperature dependency of $i_{c^{\prime}}$ several possible reasons have to be considered. As explained in Chapter 2.3, $i_{\mathrm{c}}$ indicates the transition between the two dominating diffusion processes. Before $i_{\mathrm{c}}$ is reached, the termination is dominated by the translational diffusion (TD). Afterwards, the macroradicals are long enough, and thus segmental diffusion (SD) becomes rate determining. Therefore, the chain flexibility should have an influence on $i_{c}$, and hence the size of the ester side chain affects $i_{\mathrm{c}}$. For DMA, the inflection point is at $319 \mathrm{~K}$ whereas it is $305 \mathrm{~K}$ for 2-EHMA. The long ester side chain of DMA is stiffer at low temperature, 
and thus SD is more restricted and only becomes dominant after reaching a higher chain length of the macroradicals. With increasing temperature, the ester side chain becomes more flexible, and thus SD becomes easier even at smaller chain length of macroradicals. It might be that for BMA and PnMA such a temperature dependency of $i_{\mathrm{c}}$ could also be observed at lower temperatures but it is not possible to measure these monomers at lower temperatures because of their melting points.

The fact that such a temperature dependency was not observed in previous studies for acrylates underlines the influence of the methyl group of the methacrylates on the chain flexibility, and thus on $i_{\mathrm{c}}$ (Table 4.5). ${ }^{[42]}$ Compared to acrylates, the rotation of the polymeric backbone of methacrylate is hindered due to the methyl group. Thus, methacrylates are less flexible in comparison to acrylates and the temperature dependency of $i_{\mathrm{c}}$ is more pronounced for methacrylates. Nonetheless, it has to be mentioned that the acrylates were investigated in solution which might have an influence on $i_{\mathrm{c}}$.

Another reason might be that the power law exponents $\alpha_{\mathrm{s}}$ and $\alpha_{1}$ change with increasing temperature. These two exponents are determined from the slope of the linear fits of the two regions, as explained for PnMA in Chapter 4.2. Thus, if $\alpha_{\mathrm{s}}$ is higher and $\alpha_{1}$ lower at low temperatures, the intersection, and thus $i_{\mathrm{c}}$ would be automatically higher. However, as can be seen in Figure 4.11, both composite exponents are insensitive to temperature. The overall arithmetic mean values $\alpha_{\mathrm{s}}=0.63$ for DMA and $\alpha_{\mathrm{s}}=0.61$ are shown as full and dotted line, respectively. Both values are in full agreement with the ones reported in the study of Sörensen (Table 4.2) ${ }^{[55]}$ Moreover, the overall arithmetic mean value for $\alpha_{1}$ for both monomers is $\alpha_{1}=0.16$ and deviates slightly from the ones reported in the previous studies (Table 4.2) but they are within the experimental accuracy.

Finally, the theory suggests that $i_{\mathrm{c}}$ is a fixed point where the dominating diffusion process suddenly changes. However, under real conditions, $i_{\mathrm{c}}$ should be treated more as a region than as a fixed point. Therefore, the errors are predicted higher than usual. Nonetheless, under expectation of a $i_{\mathrm{c}}$ region within the errors, the temperature dependency 


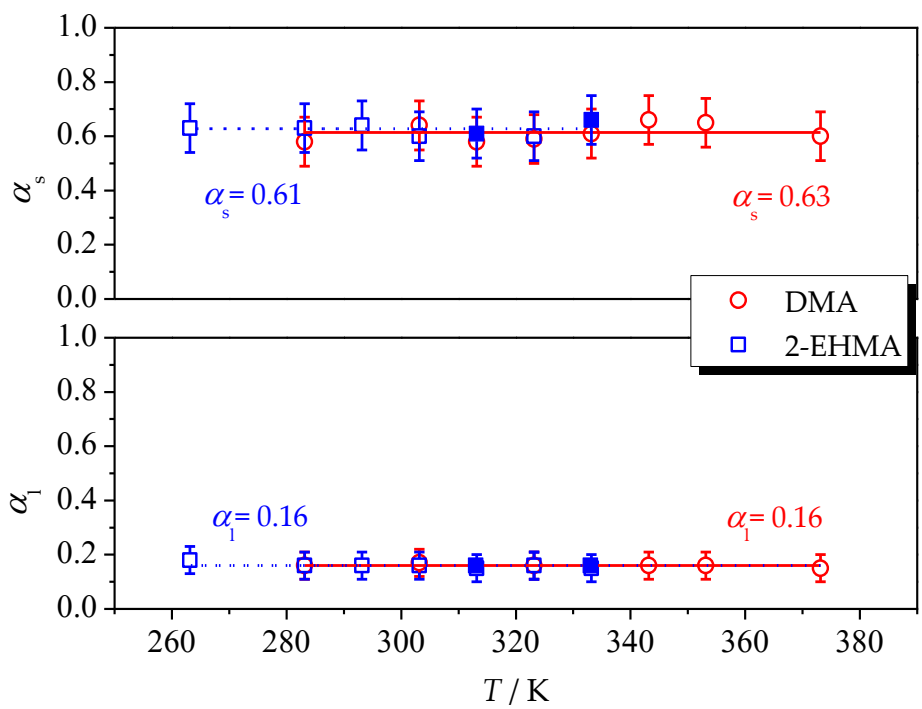

Figure 4.11: Power-law exponent $\alpha_{\mathrm{s}}$ for short-chain radicals and $\alpha_{1}$ for long-chain radicals in bulk DMA polymerization at different temperatures. The full squares refer to experimental data which have to be measured again because of experimental problems. The dashed lines indicate that both power-law exponents are not sensitive toward temperature.

of $i_{\mathrm{c}}$ is significant and for further information more measurements are required.

\subsection{Conclusion and Further Perspectives}

To sum up this chapter, the chain-length dependent termination kinetics for PnMA bulk polymerization was determined by the powerful SP-PLP-EPR method. The obtained power law exponents $\alpha_{\mathrm{s}}=0.56 \pm 0.08$ and $\alpha_{1}=0.16 \pm 0.04$ perfectly agree with the reported ones for other methacrylates and other monomers as well as with the theoretical predicted values. Moreover, the crossover chain length $i_{\mathrm{C}}=67 \pm 10$ is close to the $i_{\mathrm{c}}$ for tert-BMA $\left(i_{\mathrm{c}}=70 \pm 15\right)^{[31]}$ and a significant temperature dependency could not be observed. The activation energy for the termination rate coefficient of two monomeric radicals $E_{\mathrm{A}}\left(k_{\mathrm{t}}^{1,1}\right)=12 \mathrm{~kJ} \cdot \mathrm{mol}^{-1}$ perfectly agrees with $E_{\mathrm{A}}\left(\eta^{-1}\right)$ which was also 
observed for other methacrylates and monomers. Therefore, the product $k_{\mathrm{t}}^{1,1} \cdot \eta$ is insensitive towards temperature and can be related to the monomer structure. In comparison with other methacrylates and monomers, $k_{\mathrm{t}}^{1,1} \cdot \eta$ for PnMA fit into the tendency that $k_{\mathrm{t}}^{1,1} \cdot \eta$ decreases with increasing size of ester side chain which is caused by the increasing hydrodynamic radii.

Furthermore, one major aspect of this work was the crossover chain length $i_{\mathrm{c}}$ of DMA and 2-EHMA. A strong temperature dependency of $i_{\mathrm{c}}$ could be observed for both monomers. In contrast to the study of Sörensen, ${ }^{[55]}$ a sigmoidal decrease of $i_{\mathrm{c}}$ with increasing temperature for DMA and 2-EHMA was observed and not an exponential behavior. This discrepancy can be explained by the numerous measured data points and smaller temperature steps within this work. However, the temperature dependency of $i_{\mathrm{c}}$ is significant and clearly depends on the monomer structure. With increasing ester side chain length, the inflection point of the sigmoidal curve is shifted from $305 \mathrm{~K}$ for 2-EHMA to $319 \mathrm{~K}$ for DMA.

Nevertheless, the reason behind this temperature dependency could not be revealed so far. Further measurements are required. First investigations into the termination kinetics of hexyl methacrylate $(\mathrm{HMA})^{[56]}$ showed a temperature dependency of $i_{\mathrm{c}}$ (Figure 4.12). These values were only obtained for one sample at each temperature, and thus more measurements are necessary. Further investigations into the temperature dependency of $i_{\mathrm{c}}$ for HMA would be interesting because it seems that only a sterically not demanding side chain in the ester side chain leads to significant changes in comparison to 2-EHMA. More information could be obtained by measurements of DMA in solution. For $15 \mathrm{wt} . \%$ DMA in MeCN, also a temperature dependency of $i_{\mathrm{c}}$ was observed in the study of Sörensen ${ }^{[55]}$ In contrast, investigations into the chain-length dependent termination of poly(ethylene glycol) methyl ether methacrylate (PEGMA) in aqueous solution at $293 \mathrm{~K}$ have shown that $i_{\mathrm{c}}$ decreases from $i_{\mathrm{c}}=200 \pm 80$ for $70 \mathrm{wt} \%$ over $i_{\mathrm{c}}=150 \pm 70$ for $50 \mathrm{wt} . \%$ to $i_{\mathrm{c}}=65 \pm 20$ for $30 \mathrm{wt} . \% .{ }^{[111]}$ Because the investigations into the termination kinetics of PEGMA were performed solely at $293 \mathrm{~K}$, a temperature dependency of $i_{\mathrm{c}}$ is not known for this monomer. Thus, 


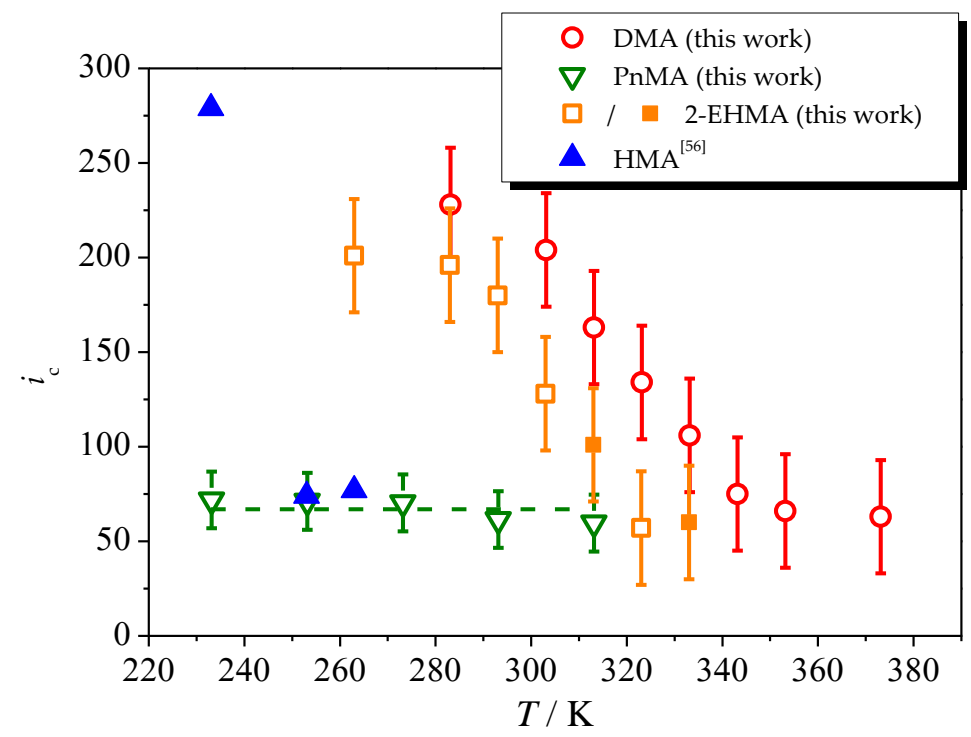

Figure 4.12: Crossover chain length $i_{\mathrm{c}}$ for bulk polymerizations of different methacrylates (PnMA green triangles, HMA blue triangles, 2-EHMA orange squares, DMA red circles) for several temperatures. According to 2-EHMA, the full squares refer to experimental data, which have to be measured again because of experimental problems. The green dashed line is the arithmetic mean value overall temperatures for PnMA $\left(i_{\mathrm{c}}=67 \pm 10\right)$.

investigations of DMA in toluene at $273 \mathrm{~K}$ could lead to deeper understanding of the temperature dependency of $i_{\mathrm{C}}$.

All in all, the results agree with the literature and previous studies. Hence, although important parts of the experimental setup were replaced or repaired, the obtained results are valid and reliable. Even the change of the laser type does not influence the termination kinetics, although it led to glass radicals during the first experiments (see Chapter 3.3.1). 


\section{KINETICS OF RADICAL COPOlYMERIZATIONS}

Since the polymerization of two types of monomers combines the properties of the related homopolymers in one copolymeric product, the great importance of copolymers is obvious. Moreover, the applications of these copolymers are wide. For instance, they are used as binder resin in automotive coating, rubber compounds, textiles and foils. ${ }^{[3,49]}$

Therefore, the interest into the kinetics of radical copolymerization is enormous because its exact knowledge opens up the access to precisely tailored high-performance products. The extensive research interest is also demonstrated by the countless studies into the propagation kinetics of radical copolymerizations..$^{[11,12,21-23,13-20]}$ As mentioned in Chapter 2.5, the propagation kinetics of radical copolymerization was investigated over decades because of the high complexity of the kinetics of radical copolymerization..$^{[5]}$

However, the high uncertainty of the copolymerization parameters obtained by fitting $k_{\mathrm{p}, \text { copo }}$ was demonstrated, too. ${ }^{[52]}$ Moreover, Heuts et al. showed that the so-obtained copolymerization parameters cannot describe the styrene radical fraction for different feed fractions of styrene for the copolymerization of styrene and MMA. ${ }^{[12]}$ Heuts et al. calculated the styrene radical fraction from stationary experiments using a transfer agent. ${ }^{[112]}$ Within this work, the styrene radical fraction is directly determined by simulations of the spectra of the styrene-d8-MMA copolymerization for different styrene feed fractions.

Moreover, Davis named the copolymerization of styrene and MMA the "fruit-fly" because of the high number of investigations into the propagation kinetics. ${ }^{[86]}$ Furthermore, the chain-length dependent termination kinetics was investigated only for the styrene-MMA copolymerization. ${ }^{[53]}$ In this study, $k_{\mathrm{t}}^{1,1}$ was obtained from stationary experiments analyzed by size exclusion chromatography. It was observed that $k_{\mathrm{t}, \mathrm{copo}}^{1,1}$ of the radical copolymerization is significantly higher than $k_{\mathrm{t}}^{1,1}$ of the corresponding homopolymerizations. ${ }^{[53]}$ Therefore, the 
copolymerization of styrene and MMA is a perfect candidate for first investigations into the chain-length dependence termination kinetics of copolymerization using the SP-PLP-EPR method. Within this chapter, the presented results are based on a previous work of the author. ${ }^{[113]}$ Moreover, another advantage of this copolymerization system is that fully deuterated species of both monomers are commercially available. This simplifies the spectra for the homopolymerizations as will be explained in this chapter. The chain-length dependent termination of the radical copolymerization of styrene and MMA was determined via PREDICI ${ }^{\circledR}$ simulations under consideration of different assumptions. Hence, first insights into the chain-length dependent termination kinetics of radical copolymerizations were obtained via SP-PLP-EPR.

\subsection{EPR Spectra of Homopolymerizations and Copolymerizations}

For the investigations into the chain-length dependent termination kinetics of radical copolymerizations, it is essential to clearly assign the different EPR signals recorded during the radical copolymerization to the two possible terminal units. Normally, the spectrum of a copolymerization is a superposition of both homopolymerization spectra. ${ }^{[14,115]}$ Nonetheless, the penultimate unit can influence the hyperfine coupling constants which might cause differences in the copolymerization spectrum compared to the superposition of the corresponding homopolymerizations. ${ }^{[84,85,116]}$ Therefore, the spectrum of both homopolymerizations will be shown and explained in the further part. Afterwards, the spectra recorded during the radical copolymerization of styrene-d8 and MMA will be compared to the superimposed spectrum of the corresponding homopolymerization or to the spectra of homopolymerizations. Thus, a clear assignment of the different signals is achieved. 


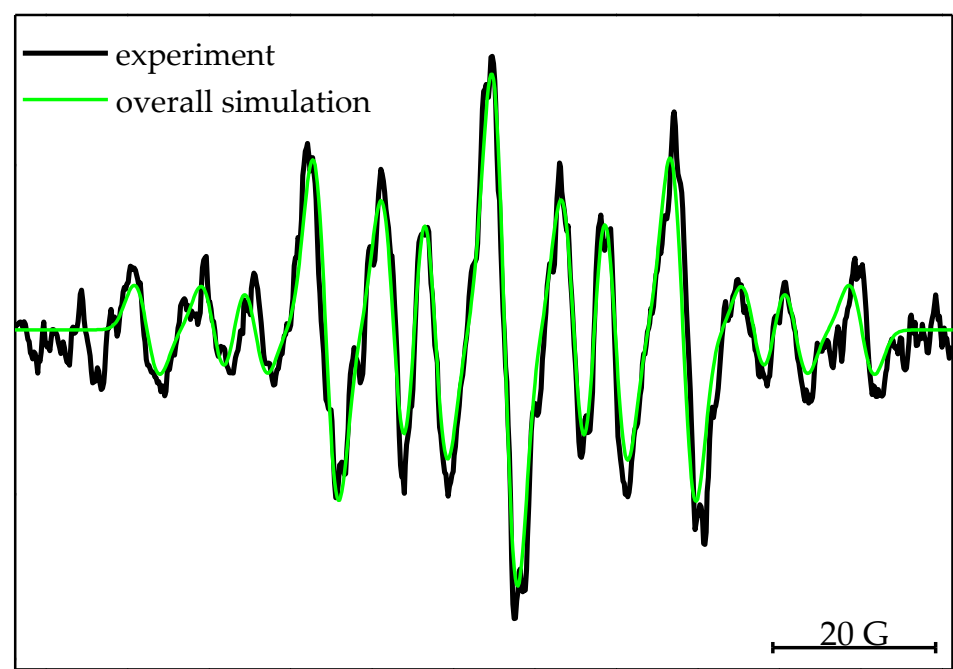

Figure 5.1: Experimental spectrum (black) recorded during the homopolymerization of MMA in bulk at $333 \mathrm{~K}$. The radicals were produced under continuous irradiation with a mercury lamp. The overall simulated spectrum is shown in green.

Table 5.1: Hyperfine coupling constants for MMA at $333 \mathrm{~K}$ determined by Matlab ${ }^{\circledR}$ simulation.

\begin{tabular}{ccc}
\hline & Conformer A & Conformer B \\
\hline \hline $\begin{array}{c}\text { Amount and position } \\
\text { of hydrogen }\end{array}$ & $a_{\mathrm{hf}} / \mathrm{G}$ & $a_{\mathrm{hf}} / \mathrm{G}$ \\
$\mathbf{1} \mathbf{H}_{\beta, 1}$ & 14.0 & 23.4 \\
$\mathbf{1} \mathbf{H}_{\beta, 2}$ & 8.8 & 0.2 \\
$\mathbf{3} \mathbf{H}_{\text {methyl }}$ & & 22.2 \\
\hline
\end{tabular}

In Figure 5.1, the spectrum recorded during a radical homopolymerization of MMA in bulk at $333 \mathrm{~K}$ under irradiation with a mercury lamp is shown. This thirteen-line spectrum is characteristic for methacrylates and results from two coexisting conformers as explained in Chapter 4.1 in detail. In comparison to the spectrum recorded during the PnMA homopolymerization at 248 K (see Figure 4.2), the hyperfine 
Table 5.2: Hyperfine coupling constants for styrene-H8 at $248 \mathrm{~K}$ used for the Simfonia ${ }^{\circledR}$-simulations.

\begin{tabular}{cccc}
\hline $\begin{array}{c}\text { Amount and position of } \\
\text { hydrogen }\end{array}$ & $\boldsymbol{a}_{\mathrm{hf}} / \mathrm{G}$ & $\begin{array}{c}\text { Amount and position of } \\
\text { hydrogen }\end{array}$ & $\boldsymbol{a}_{\mathrm{hf}} / \mathrm{G}$ \\
\hline \hline $\mathbf{1} \mathbf{H}_{\alpha}$ & 17.6 & $2 \mathrm{H}_{\text {meta }}$ & 1.7 \\
$\mathbf{2} \mathbf{H}_{\beta}$ & 16.3 & $1 \mathrm{H}_{\text {para }}$ & 5.3 \\
$\mathbf{2} \mathbf{H}_{\text {ortho }}$ & 5.2 & & \\
\hline
\end{tabular}

coupling constants differ slightly (Table 5.1 in comparison to Table 4.1) which may result from the different temperatures. Moreover, the intensity of inner lines of the MMA spectrum which refers solely to conformer A are higher as for PnMA (Figure 4.2). This is caused by the increasing rotation around the $\mathrm{C}_{\alpha}-\mathrm{C}_{\beta}$-bond with increasing temperature. Thus, the fraction of conformer A is 0.8 for MMA at $333 \mathrm{~K}$, whereas it is 0.5 for PnMA at $248 \mathrm{~K}$.

The second monomer of the investigated copolymerization is styrene. The EPR spectrum recorded during a radical homopolymerization of styrene at $248 \mathrm{~K}$ under continuous irradiation with a mercury lamp shows thirteen lines and is quite complex (black line in Figure 5.2). However, this spectrum is distinctive because the radical is delocalized over the whole the $\pi$-system of styrene. Thus, besides the hyperfine coupling to the vinylic hydrogen atoms, the radical also couples to each hydrogen of the phenyl ring. The simulation (red line in Figure 5.2) was obtained by the $a_{\mathrm{hf}}$ given in Table 5.2. Experimental and simulated spectrum perfectly agree and similar $a_{\mathrm{hf}}$ were found in the literature. ${ }^{[34,117,118]}$

Nonetheless, the superposition of these two complicated homopolymerization spectra, and thus the copolymerization spectrum results in a complicated spectrum as it has been observed in a previous study of the author (see Figure 5.3). ${ }^{[113]}$ 


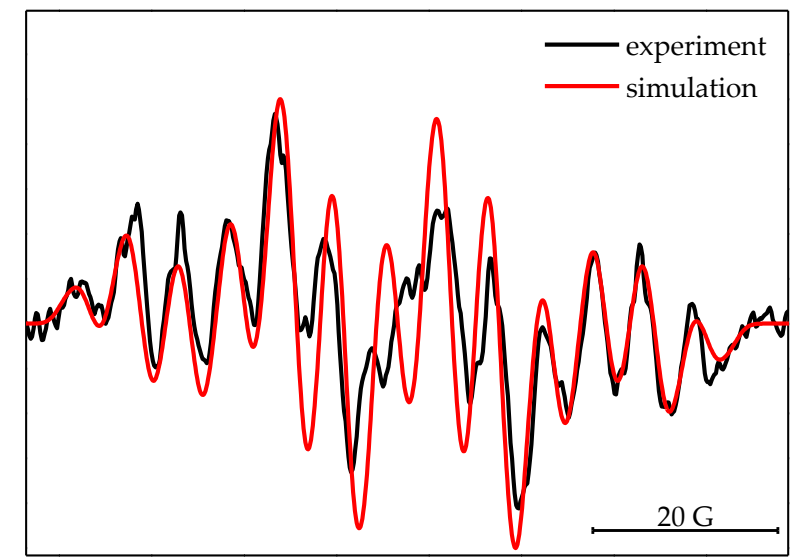

Figure 5.2: Experimental (black) and simulated (red) spectra recorded during a homopolymerization of styrene-H8 in bulk at $248 \mathrm{~K}$. The radicals were produced under continuous irradiation with a UV-lamp. The simulated spectra with the $a_{\mathrm{hf}}$ given in Table 5.2 is shown in red. ${ }^{\mathrm{ii}}$

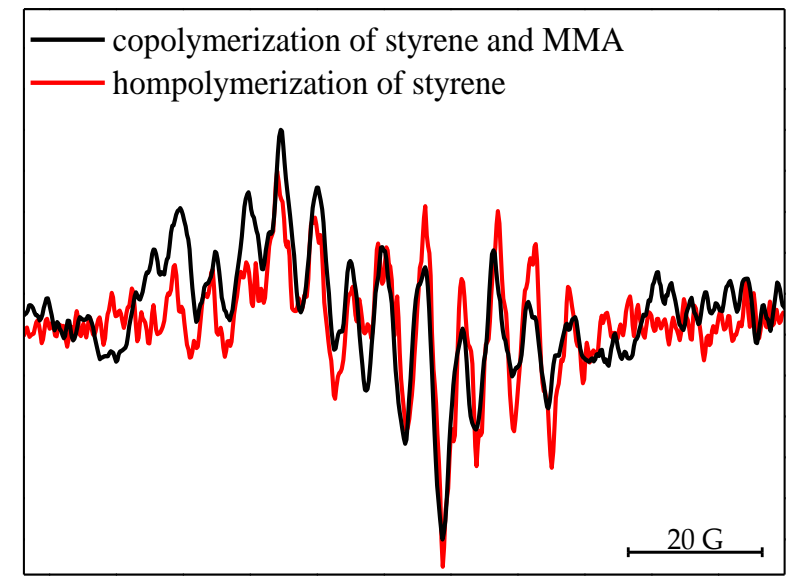

Figure 5.3: Experimental spectrum (black) recorded during the radical copolymerization of styrene and MMA $\left(f_{\text {sty }}=0.498\right.$ and $\left.f_{\mathrm{MMA}}=0.502\right)$ in bulk at $333 \mathrm{~K}$. In comparison, the spectrum recorded during the radical homopolymerization of styrene is shown in red. ${ }^{\text {ii }}$

ii The spectra were taken from the master thesis of the author where the simulation was performed with Simfonia ${ }^{\circledR}$ [ ${ }^{113]}$ 


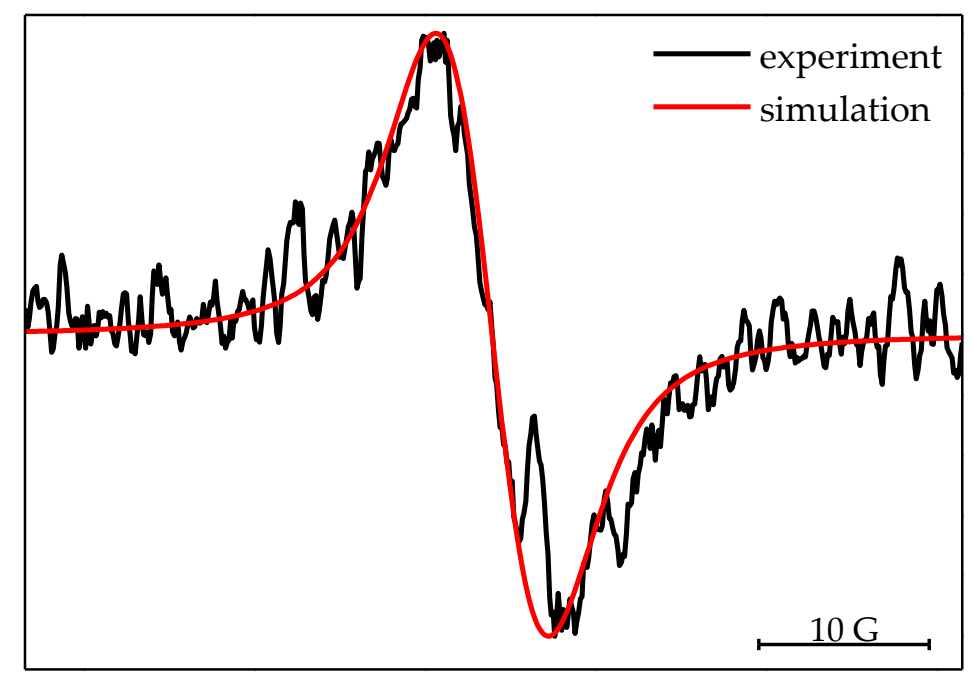

Figure 5.4: Experimental spectrum recorded during the homopolymerization of styrene-d8 in bulk at $353 \mathrm{~K}$. The radicals were produced under continuous irradiation with a UV-lamp. The simulated spectrum is shown in red.

For simplification of the copolymerization spectrum, fully deuterated styrene (styrene-d8) was used. The full deuteration simplifies the spectrum to a broad singlet (see Figure 5.4) due to the reduced gyromagnetic constant of deuterium compared to hydrogen. ${ }^{[30,34,94,119]}$ The simulated spectrum is shown in red in Figure 5.4 and is in good agreement with the experimental spectrum. Moreover, deuterated styrene was also used for the investigations into the chain-length dependent termination kinetics of styrene homopolymerizations. ${ }^{[34]}$ Furthermore, it was shown for the homopolymerizations of styrene, vinyl acetate and MMA that the deuteration does not influence the termination kinetics significantly. ${ }^{[30,34,94]}$ Additionally, investigations into the propagation kinetics of the homopolymerization of non-deuterated and deuterated styrene have shown that $k_{\mathrm{p}}$ is higher by a factor of 1.5 for the deuterated styrene compared to the non-deuterated. ${ }^{[120]}$ This will not be taken into account for $k_{\mathrm{p}}$ in the copolymerization because it is not known whether a similar effect occurs during a radical copolymerization. 


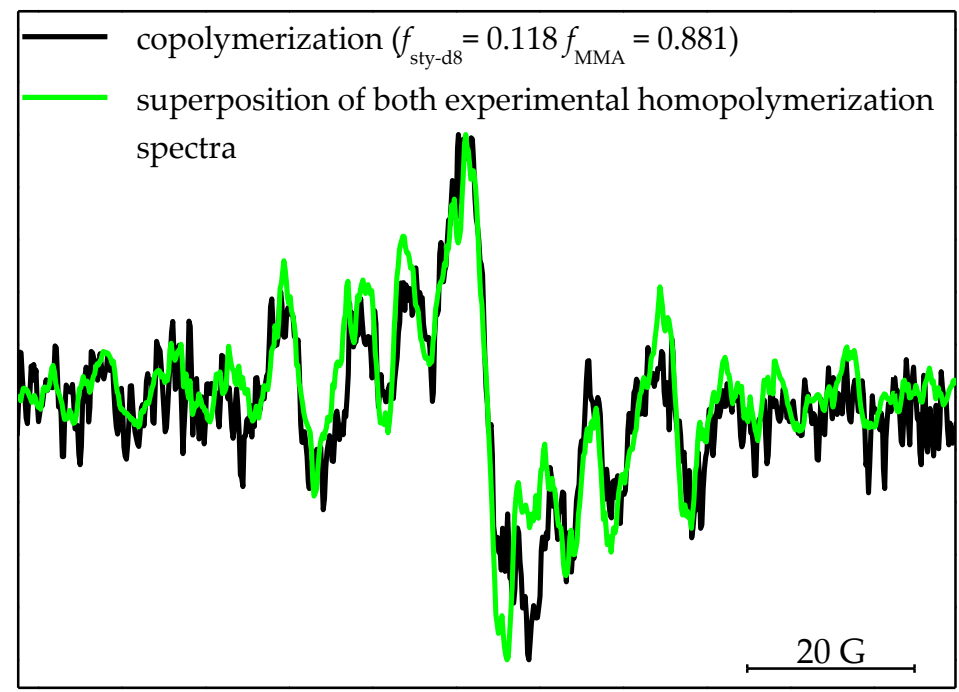

Figure 5.5: Spectrum (black) recorded during a radical copolymerization of styrene-d8 and MMA in bulk at $333 \mathrm{~K}$ for $f_{\text {sty-d } 8}=0.118$ and $f_{\text {MMA }}=0.881$. The radicals were produced under continuous irradiation with a UVlamp by the photoinitiator DCP $\left(0.5 \mathrm{~mol} \cdot \mathrm{L}^{-1}\right)$. For comparison, the superposition of the homopolymerization spectra (green) is shown. Both spectra are normalized.

In Figure 5.5, the spectra (black) recorded during the radical copolymerization of styrene-d 8 and MMA in bulk at $333 \mathrm{~K}$ for the composition with the least feed fraction of styrene-d $8\left(f_{\text {sty-d8 }}=0.118\right.$ and $f_{\text {MMA }}=0.881$ ) is shown. Furthermore, the superposition of both experimental homopolymerization spectra is depicted in green. For a better comparison, the two spectra are normalized. In comparison to the superimposed spectrum of the corresponding homopolymerization, no significant differences for the hyperfine coupling constants and the spectra in general are observed. The $a_{\mathrm{hf}}$ for the different copolymerization compositions are determined and explained in Chapter 5.2. At this point, the major aspect is the assignment of the signals to the different possible terminal units. 


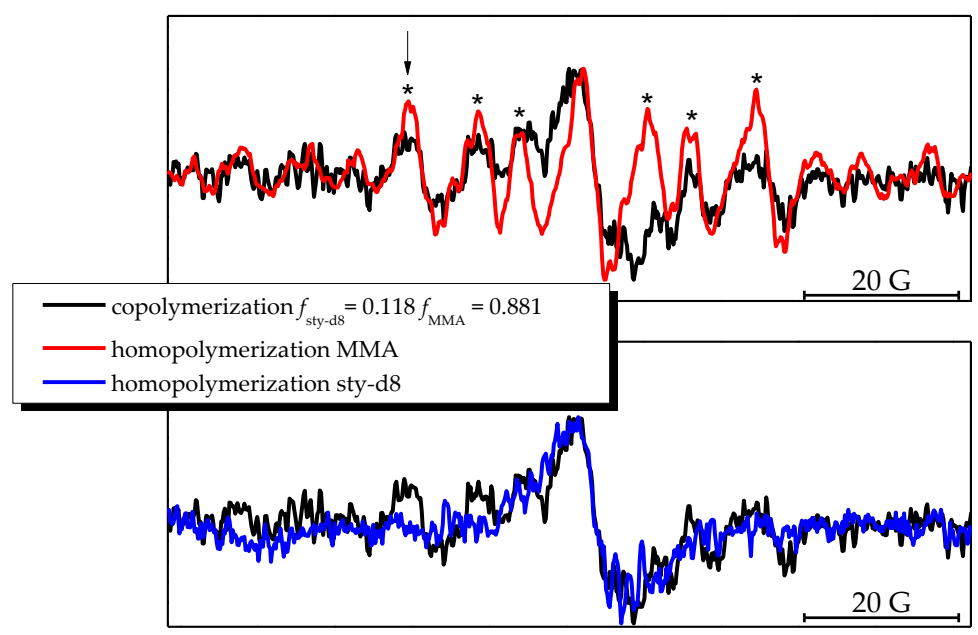

Figure 5.6: Spectrum (black) recorded during a radical copolymerization of styrene-d 8 and MMA in bulk at $333 \mathrm{~K}$ for $f_{\text {sty-d8 }}=0.118$ and $f_{\mathrm{MMA}}=0.881$. The radicals were produced under continuous irradiation with a UVlamp by the photoinitiator DCP $\left(0.5 \mathrm{~mol} \cdot \mathrm{L}^{-1}\right)$. For comparison, the spectrum (red) recorded during the radical homopolymerization of MMA and during a radical homopolymerization of styrene-d8 (blue) in bulk at $333 \mathrm{~K}$ is shown. All spectra are normalized. The arrow indicates the static field position for SP experiments of macroradicals with MMA as terminal unit.

Therefore, the copolymerization spectrum for the composition $f_{\text {sty-d8 }}=0.118$ and $f_{\mathrm{MMA}}=0.881$ is compared to the spectrum of MMA homopolymerization (red in Figure 5.6) and styrene-d8 homopolymerization (blue in Figure 5.6). As can be seen, the additional signals marked with an asterisk in the copolymerization spectrum relate to the signals of MMA as terminal unit. For further information, spectra of more compositions are required.

Thus, spectra of three more compositions were measured and are depicted in Figure 5.7. It has to be mentioned that theses spectra are normalized. It can be seen that the signal intensity related to MMA as terminal unit decreases with decreasing feed fraction of MMA (see arrow in Figure 5.7). 


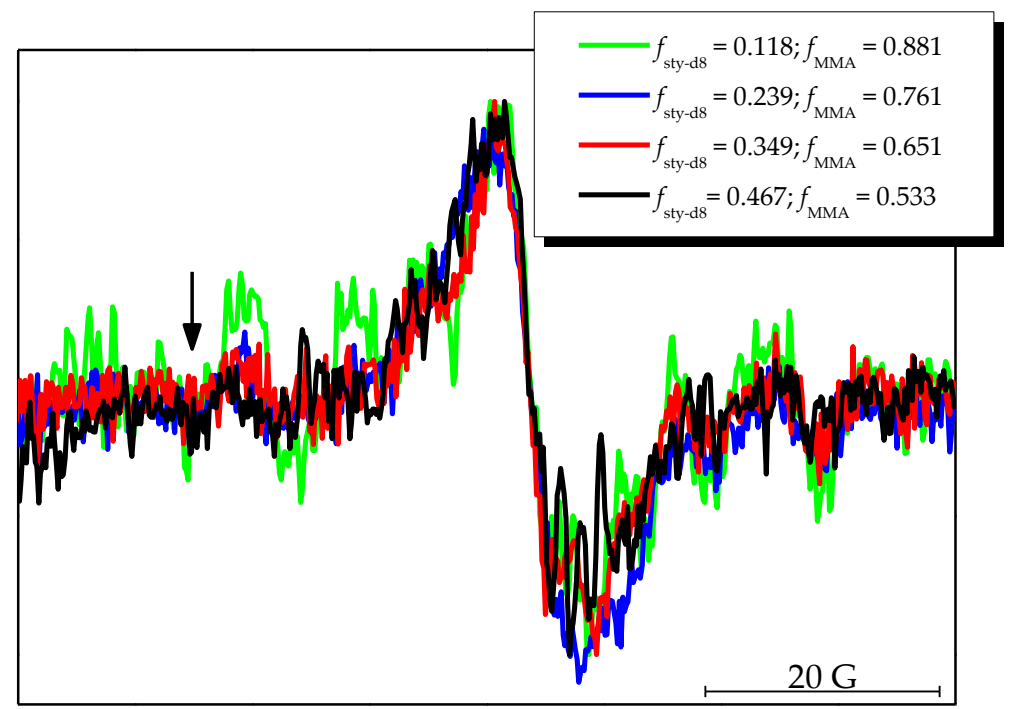

Figure 5.7: Spectra recorded during a radical copolymerizations of styrene-d8 and MMA in bulk at $333 \mathrm{~K}$ for different compositions. The radicals were produced under continuous irradiation with a UV-lamp by the photoinitiator DCP $\left(0.5 \mathrm{~mol} \cdot \mathrm{L}^{-1}\right)$. All spectra are normalized. The arrow indicates the decreasing MMA signal with decreasing MMA feed fraction.

Moreover, if the spectrum of the copolymerization with the composition near to the azeotropic point $\left(f_{\text {sty-d } 8}=0.467\right.$ and $\left.f_{\mathrm{MMA}}=0.533\right)$ is compared to the spectrum of styrene-d8 homopolymerization (Figure 5.8), only the styrene-d8 signal is observable. The signals relating to MMA as terminal unit as observed in Figure 5.6 are not recognizable.

One might argue that this is caused by formation of a monomermonomer complex. ${ }^{[121]}$ Beside the penultimate model, there is a model that assumes a monomer-monomer complex which is incorporated into the copolymer as single unit. ${ }^{[122-124]}$ Thus, the radical is localized at sty-d8 because it is stabilized by the $\pi$-system. This would result in an alternating copolymer. However, the styrene-MMA copolymerization is a statistically alternating copolymerization (Table 2.1). Moreover, Coote et al. argued against this theory because no solvent effects could be observed 


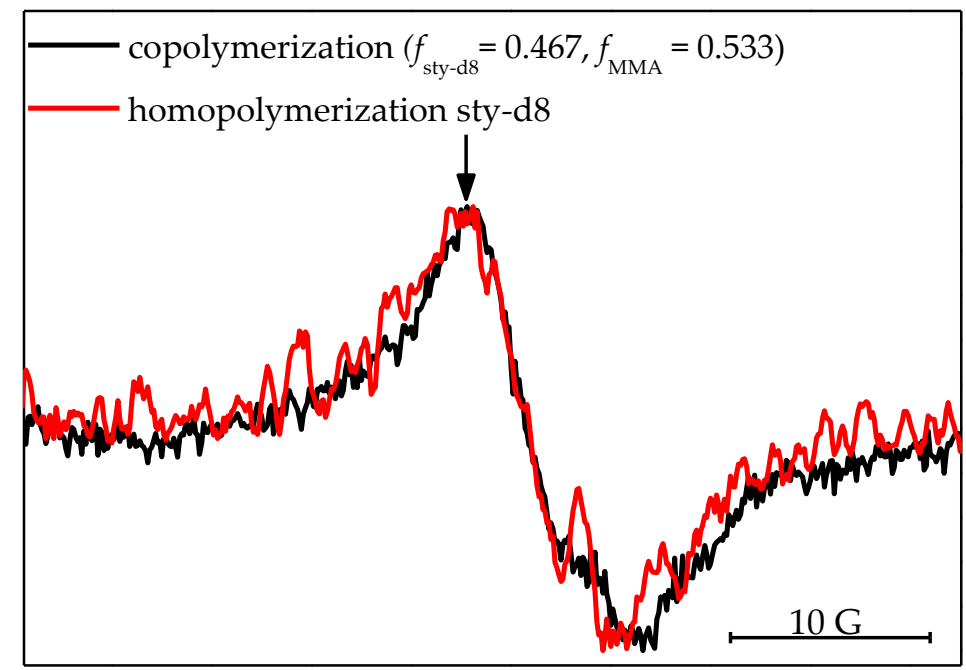

Figure 5.8: Experimental spectrum (red) recorded during a copolymerization of styrene-d8 and MMA in bulk at $353 \mathrm{~K}$. In comparison, the spectrum recorded during the homopolymerization of styrene-d8 is shown in black. The radicals were produced under continuous irradiation with a UV-lamp. The arrow indicates the static field position for the SP experiments of the copolymerization.

for this copolymerization, ${ }^{[17,125]}$ and therefore this theory is not suitable to describe the styrene-MMA copolymerization. ${ }^{[121]}$

Another model is the bootstrap model which suggests that the monomer composition differs at the radical chain end from the feed composition. ${ }^{[52,126-128]}$ The more styrene is around the radical chain end, the more styrene is incorporated and the radical concentration of MMA as terminal unit would be low. However, thermodynamic studies of the copolymerization of styrene and MMA have shown that both monomers are ideally mixed. ${ }^{[121]}$ Hence, this model is in general not a good model for the styrene-MMA copolymerization and so it cannot explain why only styrene radicals are observable for the composition $f_{\text {sty-d } 8}=0.467$ and $f_{\mathrm{MMA}}=0.533$.

Furthermore, the observation of only styrene-d8 radicals as terminal unit during the radical copolymerization with $f_{\text {sty-d } 8}=0.467$ and 


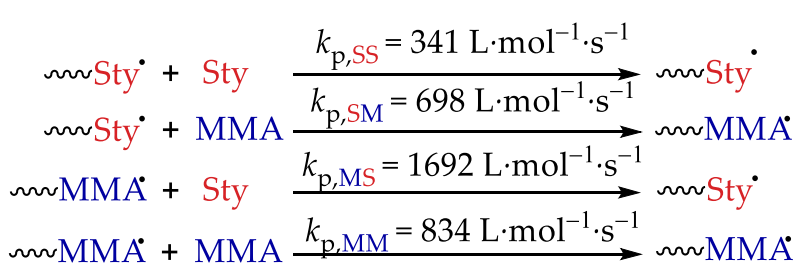

Scheme 5.1: Propagation steps of a radical copolymerization of styrene-d8 and MMA. The propagation rate coefficients of the homo-propagation at $333 \mathrm{~K}$ were taken from literature and were calculated for the crosspropagation with $r_{\mathrm{s}}=0.489$ and $r_{\mathrm{M}}=0.4929$ from literature. ${ }^{[8,9,52]}$

$f_{\mathrm{MMA}}=0.533$ can also be explained by a high cross-propagation rate. ${ }^{[121]}$ Therefore, the different propagation steps are illustrated in Scheme 5.1 and the related propagation rate coefficients are taken from literature.$^{[8,9,52]}$ For explanation, it is sufficient to use the terminal model. As can be seen, the cross-propagation step, where a MMA as terminal unit is transferred into a styrene as terminal unit, is the fastest step in this copolymerization. Hence, under stationary conditions only macroradicals with styrene- $\mathrm{d} 8$ as terminal unit might be observed.

Nonetheless, for more information, the copolymerization spectra for the different compositions were simulated, and thus the radical fraction of styrene was determined and will be discussed in the subsequent chapter.

\subsection{Determination of the Styrene Radical Fraction}

Matlab ${ }^{\circledR}$ in combination with the software package Easy Spin ${ }^{\circledR}$ was used successfully for determining MCR fractions in acrylate and acrylamide homopolymerizations. ${ }^{[39,43,129]}$ Consequently, the radical fraction of styrene $\Phi_{\mathrm{s}}$ during the copolymerization can also be obtained by this method. First, the spectra of the homopolymerizations were simulated to determine the hyperfine coupling constants (see Chapter 5.1). These hyperfine coupling constants marked the starting point for the simulation of the copolymerization spectra. Because the signal of styrene-d8 is a singlet, solely the $a_{\mathrm{hf}}$ for the macroradicals with MMA as terminal unit are interesting. For instance, the experimental 


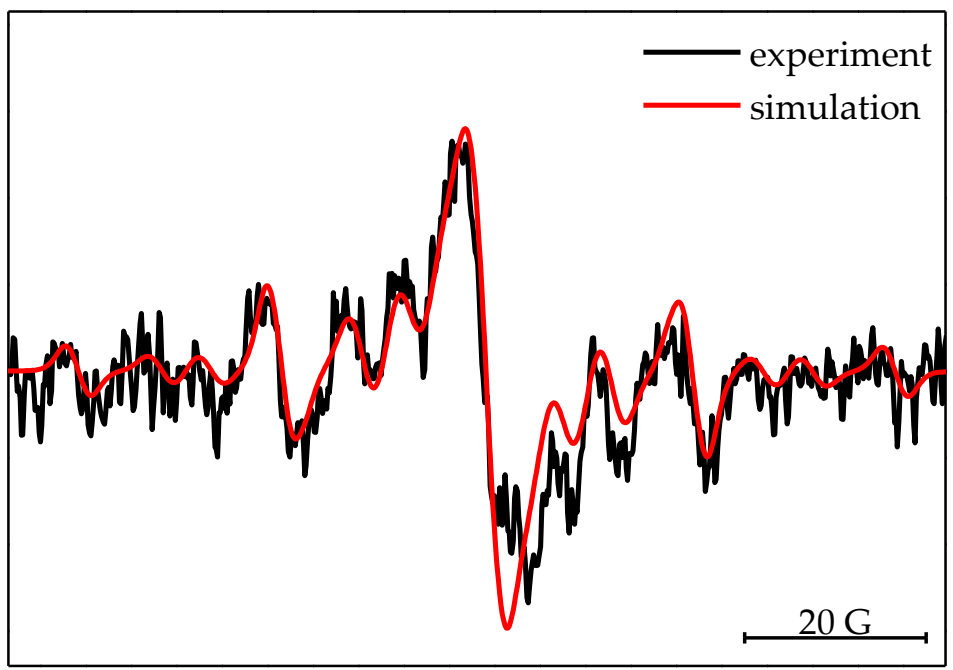

Figure 5.9: EPR spectrum recorded during a radical copolymerization of styrened8 and MMA $\left(f_{\text {sty-d8 }}=0.118\right.$ and $\left.f_{\mathrm{MMA}}=0.881\right)$ in bulk at $333 \mathrm{~K}$. The radicals were produced under continuous irradiation with a UV-lamp. The so-obtained radical fraction of styrene is $\Phi_{\mathrm{s}}=0.74 \pm 0.04$.

spectrum (black) and the simulated spectrum (red) for the copolymerization of styrene-d8 and MMA are shown Figure 5.9 for the composition with the highest feed fraction of MMA $\left(f_{\text {sty-d } 8}=0.118\right.$ and $\left.f_{\mathrm{MMA}}=0.882\right)$. As can be seen, the experimental and the simulated spectrum perfectly agree.

The determined $a_{\mathrm{hf}}$ for macroradicals with MMA as terminal unit are given in Table 5.3. Compared to the $a_{\mathrm{hf}}$ for the homopolymerization of MMA (Table 5.1), the $a_{\mathrm{hf}}$ differ slightly. This might be explained by the penultimate unit effect (PUE). In a study of Kajiwara et al., different $a_{\mathrm{hf}}$ of the two $\mathrm{H}_{\beta, 1}$ for tert-BA as terminal unit were observed if a styrene unit was the penultimate unit. ${ }^{[16]}$ Nevertheless, such differences of the $a_{\mathrm{hf}}$ were more pronounced at low temperatures $\left(-60{ }^{\circ} \mathrm{C}\right)$ and not at $60^{\circ} \mathrm{C} .^{[116]}$ Therefore, investigations at lower temperatures might bring more information about the PUE. However, for the other compositions - the experimental and simulated spectra are given in the Appendix - such a 
Table 5.3: $\quad$ Hyperfine coupling constants for macroradicals containing a MMA as terminal unit for the spectrum recorded during the radical copolymerization of styrene-d8 and MMA $\left(f_{\text {sty-d8 }}=0.118\right.$ and $\left.f_{\text {MMA }}=0.882\right)$ at $333 \mathrm{~K}$.

\begin{tabular}{ccc}
\hline & Conformer A & Conformer B \\
\hline \hline $\begin{array}{c}\text { Amount and position } \\
\text { of hydrogen }\end{array}$ & $a_{\mathrm{hf}} / \mathrm{G}$ & $a_{\mathrm{hf}} / \mathrm{G}$ \\
$\mathbf{1} \mathbf{H}_{\beta, 1}$ & 14.2 & 23.4 \\
$\mathbf{1} \mathbf{H}_{\beta, 2}$ & 8.9 & 0.2 \\
$\mathbf{3} \mathbf{H}_{\text {methyl }}$ & & 21.7 \\
\hline
\end{tabular}

difference between $a_{\mathrm{hf}}$ of the copolymerization and homopolymerization could not be observed. This is caused perhaps by the fact that intensities of the MMA signals are lower.

Beside the PUE on the $a_{\mathrm{hf}}$ further information can be obtained from the determined radical fraction of styrene $\Phi_{\mathrm{s}}$ (Table 5.4). These values were determined directly from the spectra and no further calibration was required. As can be seen, $\Phi_{\mathrm{s}}$ is always significantly higher than $f_{\mathrm{s}}$. In a preceding study of Heuts et al., $\Phi_{\mathrm{s}}$ was calculated for the copolymerization of non-deuterated styrene and MMA in bulk at $40{ }^{\circ} \mathrm{C} \cdot{ }^{[112]}$ For a better comparison, the calculated values (black circles) form literature and the experimental values (red circles) from this work are shown in Figure 5.10.

Table 5.4: $\quad$ Determined fraction of styrene-d8 radicals for different feed fractions of styrene-d8 at $333 \mathrm{~K}$.

\begin{tabular}{cc}
\hline$f_{\mathrm{s}}$ & $\boldsymbol{\Phi}_{\mathrm{s}}$ \\
\hline \hline 0 & 0 \\
0.118 & $0.74 \pm 0.04$ \\
0.239 & $0.91 \pm 0.04$ \\
0.349 & $0.96 \pm 0.04$ \\
0.467 & $0.95 \pm 0.04$ \\
1 & 1 \\
\hline
\end{tabular}




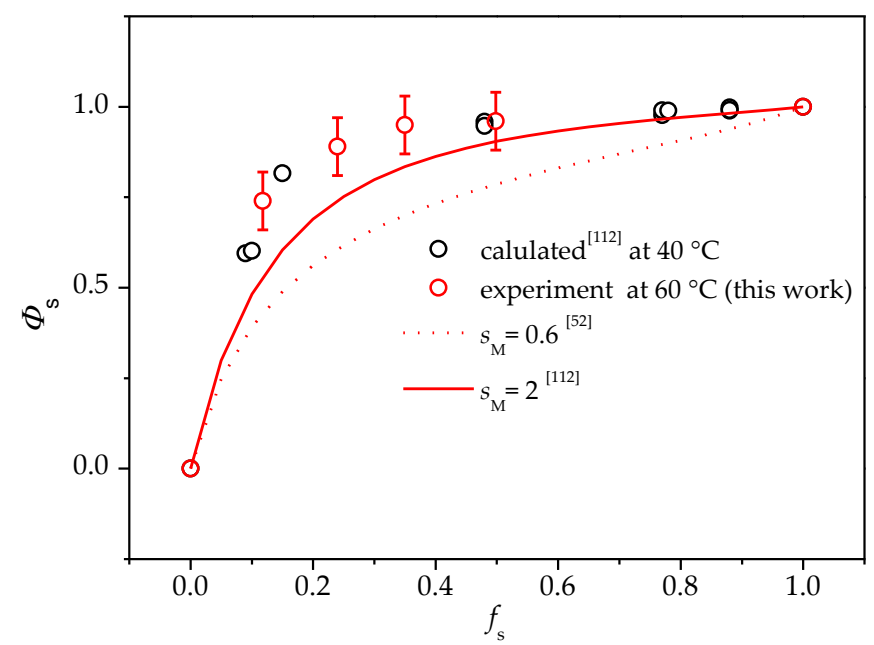

Figure 5.10: Determined radical fraction of styrene-d $8 \Phi_{\mathrm{s}}$ for different feed fractions of styrene-d $8 f_{\mathrm{s}}$ (red circles) at $333 \mathrm{~K}$. The calculated values for $\Phi_{\mathrm{s}}$ (black circles) taken from literature ${ }^{[112]}$ are given, too. Shown as red dotted line is the fit by the implicit model with $s_{\mathrm{M}}=0.6$ and as red full line with $s_{\mathrm{M}}=2$.

Although $\Phi_{\mathrm{s}}$ is determined by different methods and at different temperatures, $\Phi_{\mathrm{s}}$ of this work perfectly agrees with the previous study. Both studies show that $\Phi_{\mathrm{s}}$ is significantly higher than $f_{\mathrm{s}}$. Thus, experimental values of this work are verified by the literature values. As shown also in the literature, $\Phi_{\mathrm{s}}$ can be calculated by the following equation: ${ }^{[112]}$

$$
\Phi_{\mathrm{S}}=\frac{A_{\mathrm{SM}}}{1+A_{\mathrm{SM}}},
$$

where $A_{\mathrm{SM}}$ is the ratio of the macroradicals with styrene as terminal unit and those with MMA as terminal unit. $A_{\mathrm{SM}}$ can be calculated by the implicit penultimate model as follows: ${ }^{[12]}$ 


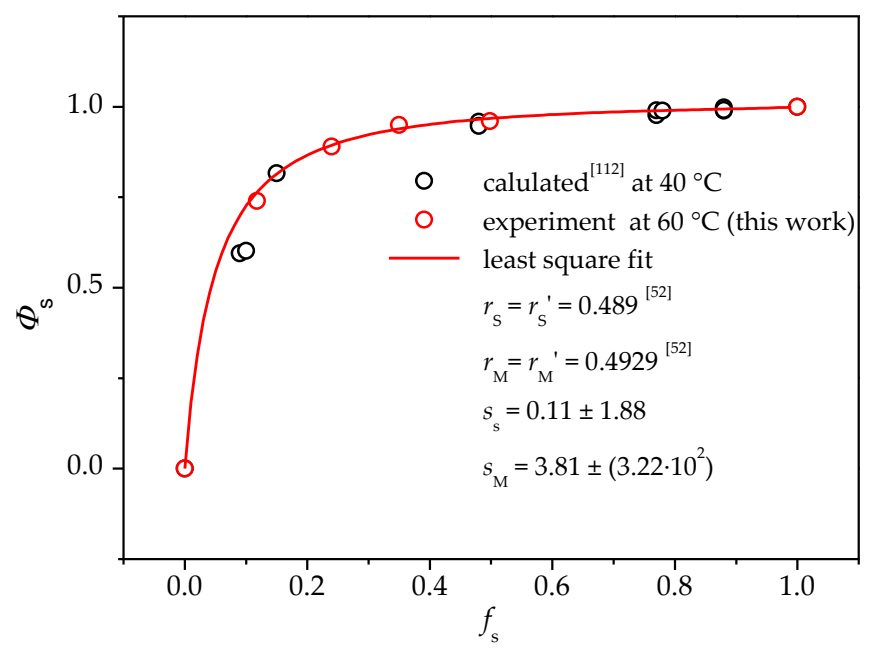

Figure 5.11: Determined radical fraction of styrene-d $8 \Phi_{\mathrm{s}}$ for different feed fractions of styrene-d $8 f_{\mathrm{s}}$ (red circles) at $333 \mathrm{~K}$. The calculated values for $\Phi_{\mathrm{s}}$ (black circles) taken from literature ${ }^{[112]}$ are given, too. The full red line represents the least-square fit based on the implicit model.

$$
A_{\mathrm{SM}}=\frac{\bar{k}_{\mathrm{p}, \mathrm{MM}} r_{\mathrm{S}} f_{\mathrm{S}}}{\bar{k}_{\mathrm{p}, \mathrm{SS}} r_{\mathrm{M}} f_{\mathrm{M}}}
$$

where $\bar{k}_{\mathrm{p}, \text { ii }}$ is given by Equation 2.35.

According to Equation 5.1 and 5.2 in conjunction with the literature known copolymerization parameters (Table 5.5), the dotted red line in Figure 5.10 is obtained. As can be seen, this fit cannot describe $\Phi_{\mathrm{s}}$ adequately. Moreover, Heuts et al. ${ }^{[112]}$ increased $s_{\mathrm{M}}=2$ which provides a better but not a perfect description of $\Phi_{\mathrm{s}}$. Thus, the uncertainty of the copolymerization parameters becomes obvious.

To obtain a better description for $\Phi_{s}$ the values for $\Phi_{\mathrm{s}}$ determined in this work were fitted by a least-squares method based on the implicit model. Therefore, $r_{\mathrm{s}}=0.489$ and $r_{\mathrm{M}}=0.4929$ were taken from literature ${ }^{[52]}$ and the s-parameters were adjusted. The result is shown in Figure 5.11. 


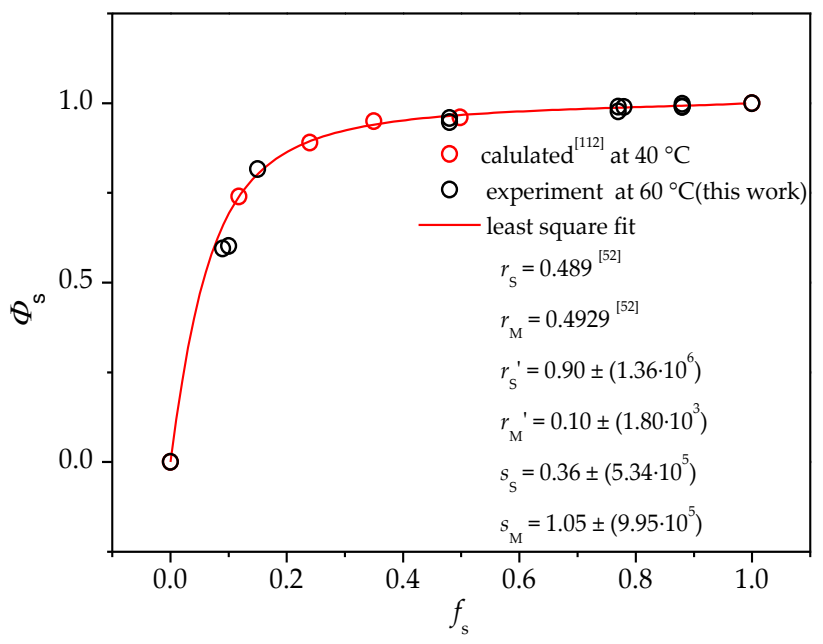

Figure 5.12: Determined radical fraction of styrene-d $8 \Phi_{\mathrm{s}}$ for different feed fractions of styrene-d $8 f_{\text {sty-d8 }}$ (red circles) at $333 \mathrm{~K}$. The calculated values for $\Phi_{\mathrm{s}}$ (black circles) taken from literature ${ }^{[112]}$ are given, too. The full red line represents the least-squares fit based on the explicit model.

The so-obtained fit represents $\Phi_{s}$ of this work acceptably. In comparison to $\Phi_{\mathrm{s}}$ from literature, ${ }^{[112]}$ the fit deviates slightly. Nevertheless, the sodetermined $s$-values have a high uncertainty.

As mentioned in Chapter 2.5.2.2, the explicit model should be preferred if possible. Considering the explicit penultimate model, $r_{\mathrm{i}}$ in Equation 5.2 can be replaced by $\bar{r}_{\mathrm{i}}$ (Equation 2.30). Hence, assuming the explicit penultimate model, $A_{\mathrm{SM}}$ is given by the subsequent equation: ${ }^{[130]}$

$$
A_{\mathrm{SM}}=\frac{\bar{k}_{\mathrm{p}, \mathrm{MM}} \bar{r}_{\mathrm{S}} f_{\mathrm{S}}}{\bar{k}_{\mathrm{p}, \mathrm{SS}} \bar{r}_{\mathrm{M}} f_{\mathrm{M}}} .
$$

Thus, the experimental $\Phi_{\mathrm{s}}$ were fitted by a least-squares fit (Figure 5.12) under consideration of Equation 5.3. This fit provides a better description of the experimental and literature values than the implicit model. However, the errors of the so-obtained copolymerization parameters are even higher. The reason of these high errors might be the 


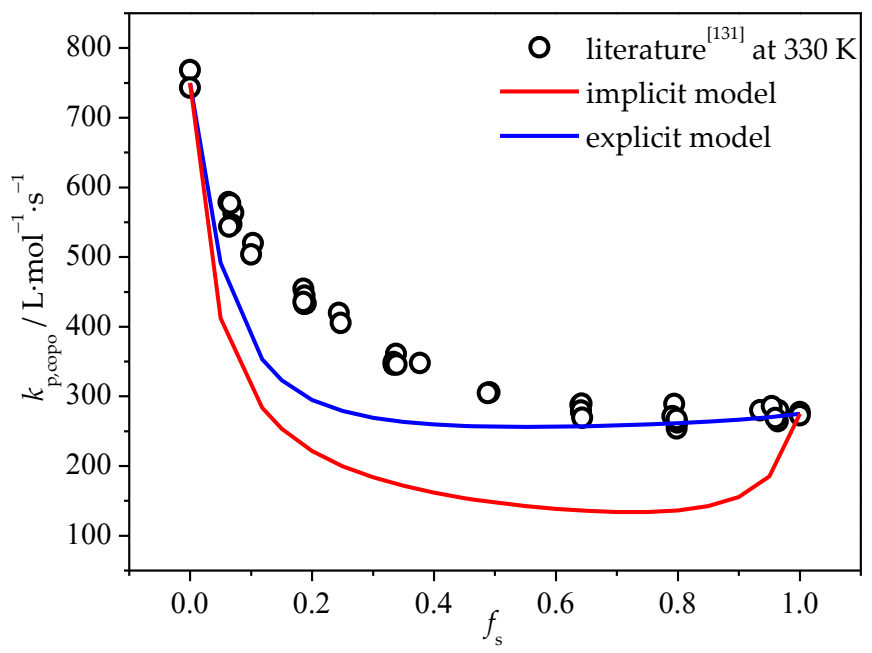

Figure 5.13: Literature values of $k_{\mathrm{p}, \text { copo }}$ (open circles) for the radical copolymerization of styrene and MMA at different feed fraction of styrene $f_{\mathrm{s}}$ at $330 \mathrm{~K} .^{[131]}$ Additionally, the fit with the copolymerization parameter obtained by the least-squares fit based on the implicit model (red) and on the explicit model (blue) is shown.

high number of adjusted parameters and indicates again the high uncertainty of the copolymerization parameters. Nevertheless, the simultaneous adjustment of all copolymerization parameters was impossible.

Moreover, it has to be verified whether the obtained copolymerization parameters the two least-square fits can describe $k_{\mathrm{p}, \text { copo }}$ and $F_{\mathrm{S}}$. Therefore, experimental data for both values for different feed fractions of styrene were taken from literature and are shown in Figure 5.13 and Figure 5.14, respectively. ${ }^{[50,131]} F_{\mathrm{S}}$ is given for $313 \mathrm{~K}$. Coote et al. could not observe a temperature dependence of the copolymerization parameters. ${ }^{[52]}$ However, they also mentioned that this can be caused by systematic errors which also indicated the uncertainty of the copolymerization parameters. ${ }^{[52]}$ 


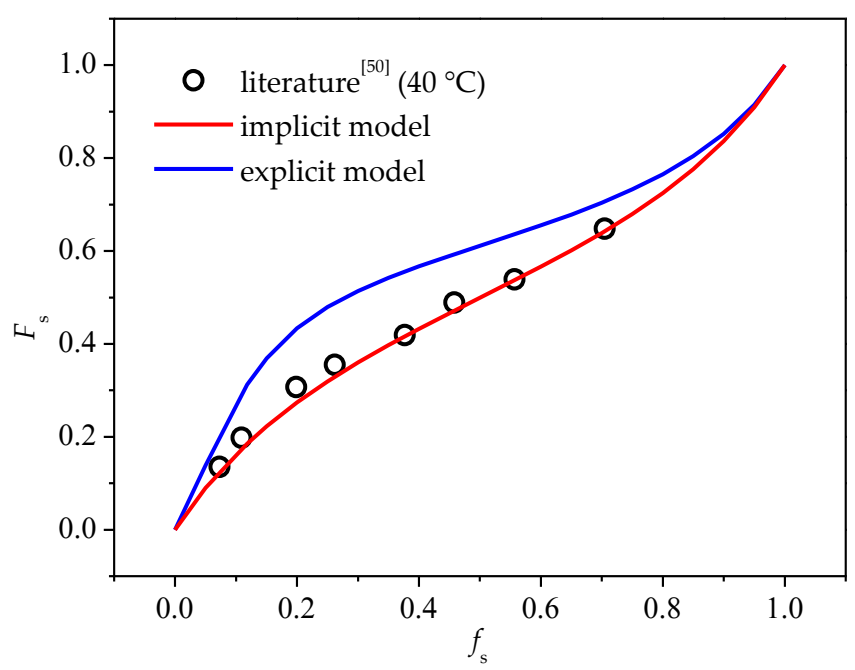

Figure 5.14: Literature values of $F_{\mathrm{s}}$ (open circles) for the radical copolymerization of styrene and MMA at different feed fraction of styrene $f_{\mathrm{s}}{ }^{[50]}$ Additionally, the fit with the copolymerization parameter obtained by the least-square based on the implicit model (red) and on the explicit model (blue) is shown.

Regarding the copolymerization parameters determined by the implicit model, a big discrepancy between experimental values for $k_{\mathrm{p}, \text { copo }}$ taken from literature and the calculated $k_{\mathrm{p}, \mathrm{copo}}$ by the implicit model are observed (red line in Figure 5.13). However, the experimental $F_{\mathrm{S}}$ is perfectly described by the implicit model (red line in Figure 5.14) which is of cause not surprisingly because $F_{\mathrm{S}}$ is only calculated by the used literature $r$-values and $r^{\prime}$-values. If the copolymerization parameters determined by the explicit model are compared with the experimental values, neither $k_{\mathrm{p}, \text { copo }}$ nor $F_{\mathrm{S}}$ are adequately represented by the copolymerization parameters obtained by the two least-square fits.

To sum up so far, the obtained $\Phi_{s}$ could not be described by literature known copolymerization parameters whereas the obtained copolymerization parameters by fitting $\Phi_{s}$ cannot adequately represent the experimental data for $k_{\mathrm{p}, \text { copo }}$ and $F_{\mathrm{S}}$ taken from literature. All this led 
Table 5.5: Copolymerization parameter obtained from manually fitting the experimental data of $\Phi_{\mathrm{s}}$ under consideration of the implicit and explicit penultimate model, respectively. In comparison, the literature values are given, too.

\begin{tabular}{ccccc}
\hline & $\begin{array}{c}\text { manual fit } \\
\text { (implicit) }\end{array}$ & $\begin{array}{c}\text { manual fit } \\
\text { (explicit) }^{-}\end{array}$ & $\begin{array}{c}\text { literature } \\
\text { (implicit) }^{[52]}\end{array}$ & $\begin{array}{c}\text { literature } \\
\text { (explicit) }^{[132]}\end{array}$ \\
\hline \hline$r_{\mathrm{S}}$ & $0.50 \pm 0.05$ & $0.4 \pm 0.1$ & 0.489 & 0.498 \\
$r_{\mathbf{M}}$ & $0.30 \pm 0.05$ & $0.30 \pm 0.05$ & 0.490 & 0.463 \\
$r_{\mathbf{S}}{ }^{\prime}$ & $0.50 \pm 0.05$ & $0.6 \pm 0.1$ & 0.489 & 0.547 \\
$\boldsymbol{r}_{\mathbf{M}}{ }^{\prime}$ & $0.30 \pm 0.05$ & $0.4 \pm 0.1$ & 0.490 & 0.589 \\
$\boldsymbol{s}_{\mathrm{S}}$ & $0.28 \pm 0.05$ & $0.30 \pm 0.05$ & 0.362 & 0.478 \\
$\boldsymbol{s}_{\mathbf{M}}$ & $5 \pm 3$ & $5 \pm 3$ & 0.602 & 0.256 \\
\hline
\end{tabular}

to the question whether the penultimate model is applicable to the styrene-MMA copolymerization. Similar discrepancies to describe $k_{\mathrm{p}, \text { copo }}$ by the terminal model revealed its invalidity (see Chapter 2.5.2.2).

To analyze whether the penultimate model can adequately describe all experimental data simultaneously $\left(\Phi_{\mathrm{s}} k_{\mathrm{p}, \text { copo }}\right.$ and $\left.F_{\mathrm{S}}\right)$, all copolymerization parameters were manually adjusted in Excel $^{\circledR}$. Therefore, each copolymerization parameter was manually adjusted in small steps for the implicit and explicit penultimate model so that the parameter sets can adequately describe all experimental data. The so-obtained copolymerization parameters are given in Table 5.5 and are compared with the literature known copolymerization parameters. As can be seen, the $s_{\mathrm{M}}$ values deviate the most from the literature values. The errors of the manually obtained copolymerization parameters were also determined manually. In the Appendix, the deviations from the manual fit are shown for the errors. Furthermore, the influence of each copolymerization parameter on the different fits could be observed.

Moreover, the fitting curves related to these copolymerization parameters are shown in Figure 5.15. As can be seen, it is possible to describe all experimental data at the same time by the implicit model (red line) as well as by the explicit penultimate model (blue line). Hence, the penultimate model is reliable. Additionally, it shows the uncertainty of 

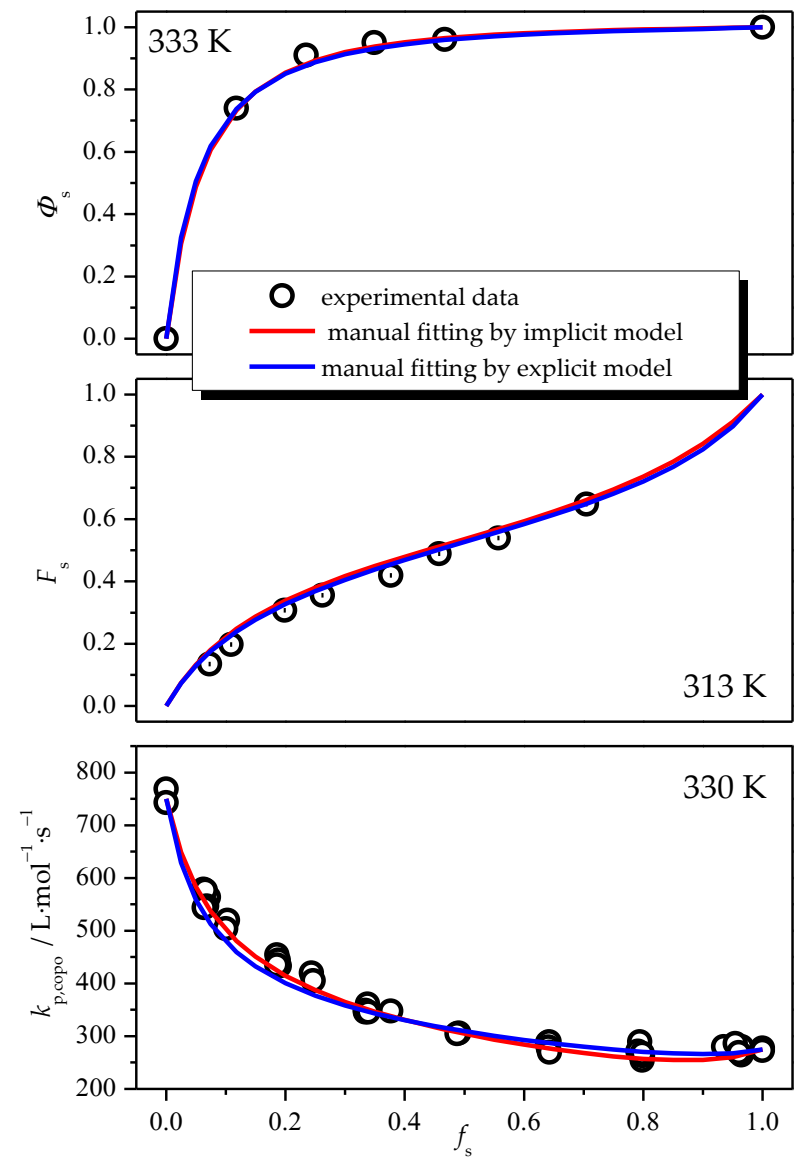

Figure 5.15: Experimental data for the radical fraction of styrene $\Phi_{s^{\prime}}$ the molar fraction of styrene in the copolymer $F_{\mathrm{S}}{ }^{[50]}$ and the overall $k_{\mathrm{p}, \text { copo }}{ }^{[131]}$ depending on the feed fraction of styrene $f_{\mathrm{s}}$. The manually fitted curves are shown in red for the implicit and in blue for the explicit penultimate model. The so-obtained copolymerization parameters are given in Table 5.5 .

the copolymerization parameters which has been obtained so far by only fitting the experimental data $k_{\mathrm{p} \text {,copo }}$ and $F_{\mathrm{S}}$. Thus, the additional information of $\Phi_{\mathrm{s}}$ can lead to more precise copolymerization parameters. 
Which of the both penultimate models should be preferred, is not easy to answer. On the one hand, the implicit penultimate model perfectly describes $\Phi_{\mathrm{s}}$ and $k_{\mathrm{p}, \text { copo }}$ whereas it leads to small differences in $F_{\mathrm{s}}$. On the other hand, the explicit model can entirely describe $F_{\mathrm{S}}$ and $k_{\mathrm{p}, \text { copor }}$ but small differences are obtained for $\Phi_{\mathrm{s}}$. Nonetheless, it exists more evidence which supports the explicit penultimate model more than the implicit model. ${ }^{[81-83]}$ Therefore, the explicit model should be also preferred here. Moreover, simultaneous fitting of all experimental data may provide more precise copolymerization parameters.

\subsection{SP Experiments}

For determining the chain-length dependent termination kinetic of the radical copolymerization of styrene and MMA $\left(f_{\text {sty-d } 8}=0.498\right.$ and $\left.f_{\text {MMA }}=0.502\right), \quad$ SP experiments were performed at $333 \mathrm{~K}$. The concentration-time profile is shown in Figure 5.16 and was measured during the master thesis of the author. ${ }^{[113]}$ Moreover, up to 60 individual concentration-time profiles were co-added. The monomer-to-polymer conversion was determined gravimetrically and was found below $5 \%$.

As can be seen, the decay of the concentration-time profile is fast which is why the smallest time resolution $(10 \mathrm{~ms})$ had to be chosen. During the master thesis of the author, it was examined whether this fast decay results from oxygen or inhibitor. ${ }^{[113]}$ None of those caused this fast decay. Furthermore, although the MMA signals are not observable in the spectrum, it might be possible that the decay of macroradicals containing MMA as terminal unit are measurable by SP experiments. Therefore, a static field position for MMA was chosen from spectrum in Figure 5.6 (indicated with an arrow). Nonetheless, it was impossible to measure SP experiments of the MMA signal at the static field position. This might be caused by the fact that the signal-to-noise ratio was low and the concentration of macroradicals containing MMA as terminal unit was too low.

Moreover, determining the chain-length dependent termination kinetics of copolymerizations is not as easy as for homopolymerizations (Chapter 4.2) because the terminal unit, and thus the reactivity of it 


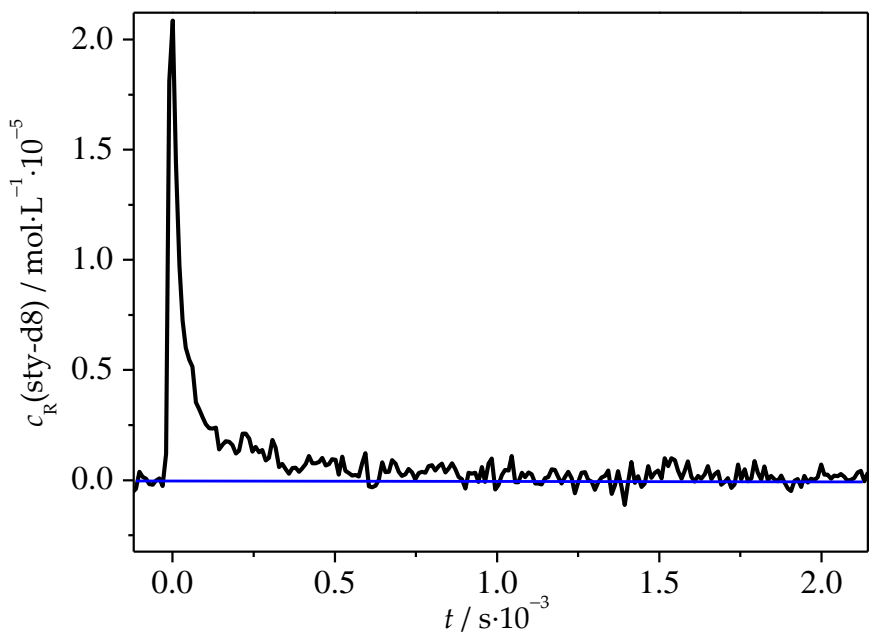

Figure 5.16: Experimental concentration-time profile for the radical copolymerization of styrene-d 8 and MMA $\left(f_{\text {sty }}=0.498\right.$ and $\left.f_{\text {MMA }}=0.502\right)$ in bulk at $333 \mathrm{~K}$. The static field position is indicated with an arrow in Figure 5.8.iii

changes during the copolymerization. Therefore, the equation for the chain length (Equation 2.44) cannot be assumed for copolymerizations. Thus, for data evaluation of the copolymerization, the commercially available simulation program PREDICI ${ }^{\circledR}$ was used.

\subsection{PREDICl ${ }^{\circledR}$ Simulations}

In comparison to the previous work of the author, ${ }^{[113]}$ the PREDICI ${ }^{\circledR}$ model was refined. Within this work, the implicit model was considered whereas only the terminal model was considered in the previous work. Therefore, the propagation rate coefficient for the homo-propagations were calculated by Equation 2.35.

iii The SP experiment of the copolymerization was taken from the master thesis of the author. ${ }^{[113]}$ 
Table 5.6: Literature values for $k_{\mathrm{p}}$ and $\alpha_{\mathrm{s}}$ for the homopolymerizations and the copolymerization parameters used within the PREDICI ${ }^{\circledR}$ simulations.

\begin{tabular}{ccc}
\hline & styrene & MMA \\
\hline \hline $\boldsymbol{k}_{\mathrm{p}}(333 \mathrm{~K}) /{\mathrm{L} \cdot \mathrm{mol}^{-1} \cdot \mathrm{s}^{-1}}$ & $341^{[8]}$ & $834^{[9]}$ \\
$\boldsymbol{\alpha}_{\mathrm{s}}$ & $0.51^{[34]}$ & $0.63^{[30]}$ \\
$\boldsymbol{r}$ & $0.489^{[52]}$ & $0.4929^{[52]}$ \\
$\boldsymbol{s}$ & $0.362^{[52]}$ & $0.602^{[52]}$ \\
\hline
\end{tabular}

\section{initiation}

$$
\begin{array}{lll}
\mathrm{I}^{\bullet}+\mathrm{M}_{\mathrm{S}} & \stackrel{k_{\mathrm{i}, \mathrm{S}}}{\longrightarrow} & \mathrm{R}\left(\mathrm{M}_{\mathrm{S}}^{\bullet}\right)_{1} \\
\mathrm{I}^{\bullet}+\mathrm{M}_{\mathrm{M}} & \stackrel{k_{\mathrm{i}, \mathrm{M}}}{\longrightarrow} & \mathrm{R}\left(\mathrm{M}_{\mathrm{M}}^{\bullet}\right)_{1}
\end{array}
$$

\section{propagation}

$\mathrm{R}\left(\mathrm{M}_{\mathrm{S}}^{\bullet}\right)_{i}+\mathrm{M}_{\mathrm{S}}$

$\begin{array}{lll}\stackrel{\bar{k}_{\mathrm{p}, \mathrm{SS}}}{\longrightarrow} & \mathrm{R}\left(\mathrm{M}_{\mathrm{S}}\right)_{i+1} & \text { homo-propagation } \\ \stackrel{\bar{k}_{\mathrm{p}, \mathrm{SM}}}{\longrightarrow} & \mathrm{R}\left(\mathrm{M}_{\mathrm{M}}\right)_{i+1} & \text { cross-propagation } \\ \stackrel{\bar{k}_{\mathrm{p}, \mathrm{MS}}}{\longrightarrow} & \left.\mathrm{R}\left(\mathrm{M}_{\mathrm{S}}\right)\right)_{i+1} & \text { cross-propagation } \\ \stackrel{\bar{k}_{\mathrm{p}, \mathrm{MM}}}{\longrightarrow} & \mathrm{R}\left(\mathrm{M}_{\mathrm{M}}\right)_{i+1} & \text { homo-propagation }\end{array}$

$\mathrm{R}\left(\mathrm{M}_{\mathrm{S}}\right)_{i}+\mathrm{M}_{\mathrm{M}}$

$\mathrm{R}\left(\mathrm{M}_{\mathrm{M}}\right)_{i}+\mathrm{M}_{\mathrm{S}}$

$\mathrm{R}\left(\mathrm{M}_{\mathrm{M}}^{\bullet}\right)_{i}+\mathrm{M}_{\mathrm{M}}$

$\stackrel{\kappa_{\mathrm{p}, \mathrm{MM}}}{\longrightarrow}$

$\mathrm{R}\left(\mathrm{M}_{\mathrm{M}}^{\bullet}\right)_{i+1}$

homo-propagation

\section{termination}

$$
\begin{aligned}
& \mathrm{R}\left(\mathrm{M}_{\mathrm{S}}\right)_{i}+\mathrm{R}\left(\mathrm{M}_{\mathrm{S}}^{\bullet}\right)_{j} \quad \stackrel{k_{\mathrm{t}, S \mathrm{~S}}^{1,1}, \alpha_{\mathrm{s} S \mathrm{~S}}}{\longrightarrow} \quad \mathrm{R}\left(\mathrm{M}_{\mathrm{S}} \mathrm{M}_{\mathrm{S}}\right)_{i+j} \quad \text { homo-termination } \\
& \mathrm{R}\left(\mathrm{M}_{\mathrm{S}}\right)_{i}+\mathrm{R}\left(\mathrm{M}_{\mathrm{M}}\right)_{j} \quad \stackrel{k_{\mathrm{t}, \mathrm{S}}^{1,}, \alpha_{\mathrm{s} S \mathrm{M}}}{\longrightarrow} \mathrm{R}\left(\mathrm{M}_{\mathrm{S}} \mathrm{M}_{\mathrm{M}}\right)_{i+j} \quad \text { cross-termination } \\
& \mathrm{R}\left(\mathrm{M}_{\mathrm{M}}^{\bullet}\right)_{i}+\mathrm{R}\left(\mathrm{M}_{\mathrm{M}}^{\bullet}\right)_{j} \stackrel{k_{\mathrm{t}, \mathrm{MM}}^{1,1}, \alpha_{\mathrm{s}, \mathrm{MM}}}{\longrightarrow} \mathrm{R}\left(\mathrm{M}_{\mathrm{M}} \mathrm{M}_{\mathrm{M}}\right)_{i+j} \quad \text { homo-termination }
\end{aligned}
$$

Scheme 5.2: Reaction scheme used for the PREDICI ${ }^{\circledR}$ simulations. The index $S$ refers to styrene-d8 and M to MMA, respectively.

The different reaction steps for the copolymerization of styrene and MMA considered in the PREDICI ${ }^{\circledR}$ simulations are shown in Figure 5.16. Since the chain lengths of the macroradicals $(i>10)$ during the SP experiments were below the crossover chain length of both homopolymerizations (Table 4.5), the termination reactions were only obtained by the equation for the short chain region (Equation 2.21). For $\alpha_{\mathrm{s}, \mathrm{SM}}$ of the cross-termination and $\alpha_{\mathrm{s}, \mathrm{copo}}$ the arithmetic mean value of both 
homopolymerizations was used (Table 5.6). ${ }^{[30,34]}$ Moreover, $k_{\mathrm{p}}$ for the homopolymerization and the copolymerization parameters were also taken from literature. ${ }^{[52]}$ The index $S$ refers to styrene independently whether styrene or styrene-d8 was used.

Additionally, this PREDICI ${ }^{\circledR}$ model was refined by implementing further aspects such as the influence of the viscosity on the termination kinetics, equal termination reactions, different copolymerization parameters and different chain lengths of the macroradicals. Hereinafter, four different simulation variants are presented, and the detailed refinements will be explained in the related parts.

\subsubsection{Influence of Different Termination Reactions}

As mentioned in Chapter 4.2, the viscosity significantly affects the termination. Hence, if cross-termination and homo-terminations are assumed to be different, the viscosity of the copolymerization composition has to be taken into account for both homo-terminations (simulation variant A). In Chapter 4.2, it was also shown that the product $k_{\mathrm{t}}^{1,1} \cdot \eta$ should be constant at different temperatures for the homopolymerizations of styrene and MMA (Table 4.4). Thus, the value for $k_{\mathrm{t}}^{1,1} \cdot \eta$ for the homopolymerizations are taken to calculate $k_{\mathrm{t}}^{1,1}$ for the viscosity of the copolymerization of styrene and MMA (Table 5.7). The Arrhenius plot of the fluidity for the copolymerization is given in the Appendix. The experimental data and the simulated data after parameter

Table 5.7: $\quad k_{\mathrm{t}}^{1,1} \eta$ for the homopolymerization taken from literature, the determined $\eta$ (соро) and the calculated $k_{\mathrm{t}}^{1,1}$ for the homo-termination in the copolymerization under consideration of $\eta$ (copo) (simulation variant A).

\begin{tabular}{ccc}
\hline & styrene & MMA \\
\hline \hline $\begin{array}{c}\boldsymbol{k}_{\mathrm{t}}^{1,1} \eta / \mathrm{L} \cdot \mathrm{mPa} \cdot \mathrm{mol}^{-1} \cdot \mathbf{1 0} \\
\eta(\mathbf{c o p o})\end{array}$ & 3.2 & 3.7 \\
$\boldsymbol{k}_{\mathrm{t}}^{\mathbf{1 , 1}} / \mathrm{L} \cdot \mathrm{mol}^{-1} \cdot \mathrm{s}^{-1} \cdot \mathbf{1 0}^{8}$ & 7.8 & 0.41 \\
\hline
\end{tabular}




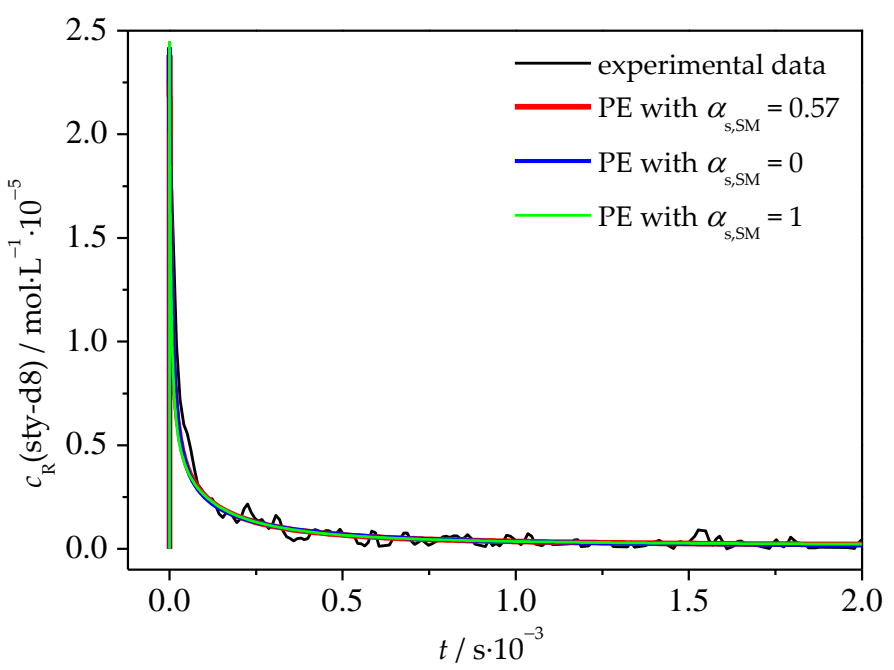

Figure 5.17: Experimental concentration-time profile for the radical copolymerization of styrene-d8 and MMA $\left(f_{\text {Sty }}=0.498\right.$ and $\left.f_{\text {MMA }}=0.502\right)$ in bulk at $333 \mathrm{~K}$. In comparison, the concentration-time profiles obtained from the parameter estimation (PE) via PREDICI ${ }^{\circledR}$ are shown considering different $\alpha_{\mathrm{s}, \mathrm{SM}}$ for simulation variant $\mathrm{A}$.

estimation (PE) are shown in Figure 5.17. Since $\alpha_{\mathrm{s}}$ is not known for the copolymerization, an error estimation is performed by inserting $\alpha_{\mathrm{s}, \mathrm{SM}}=0$ and $\alpha_{\mathrm{s}, \mathrm{SM}}=1$. The simulated concentration-time profiles adequately represent the experimental data. A significant difference between the used $\alpha_{\mathrm{s}, \mathrm{SM}}$ cannot be observed.

Beside $k_{\mathrm{t}, \mathrm{SM}}^{1,1}$, the initial radical concentrations of styrene and MMA were adjusted during the parameter estimation. The so-obtained values are given in Table 5.8. Moreover, for a better comparison, the ratio of $k_{\mathrm{t}, \mathrm{SM}}^{1,1}$ and $k_{\mathrm{t}}^{1,1}$ of the homopolymerizations are given, too. As can be seen, the obtained $k_{\mathrm{t}, \mathrm{SM}}^{1,1}$ is significantly higher than $k_{\mathrm{t}}^{1,1}$ of the corresponding homopolymerization. For $\alpha_{\mathrm{s}, \mathrm{SM}}=0.57, k_{\mathrm{t}, \mathrm{SM}}^{1,1}$ is five times higher than $k_{\mathrm{t}, \mathrm{SS}}^{1,1}$ and four times higher than $k_{\mathrm{t}, \mathrm{MM}}^{1,1}$. However, in comparison to the previous study of the author, ${ }^{[113]} k_{\mathrm{t}, \mathrm{SM}}^{1,1}$ does not differ although the viscosity and implicit penultimate model are considered within this work. 
Table 5.8: $\quad$ From the parameter estimation (simulation variant $\mathrm{A}$ ) obtained values for $c_{\mathrm{R}}^{0}$ (styrene), $c_{\mathrm{R}}^{0}$ (MMA) and $k_{\mathrm{t}, \mathrm{SM}}^{1,1}$ for different $\alpha_{\mathrm{s}}$. For comparison, the ratios $k_{\mathrm{t}, \mathrm{SM}}^{1,1} / k_{\mathrm{t}, S \mathrm{~S}}^{1,1}$ and $k_{\mathrm{t}, \mathrm{SM}}^{1,1} / k_{\mathrm{t}, \mathrm{MM}}^{1,1}$ are given, too. The results from the previous work of the author is given, too.

\begin{tabular}{|c|c|c|c|c|}
\hline & $\alpha_{\mathrm{s}, \mathrm{SM}}=0$ & $\alpha_{\mathrm{s}, \mathrm{SM}}=0.57$ & $\alpha_{\mathrm{s}, \mathrm{SM}}=1$ & $\alpha_{\mathrm{s}, \mathrm{SM}}=0.57^{[113]}$ \\
\hline $\begin{array}{l}c_{\mathrm{R}}^{\mathbf{0}}(\mathrm{sty}-\mathrm{d} 8) / \\
\mathrm{mol} \cdot \mathrm{L}^{-1} \cdot 10^{-5}\end{array}$ & 2.5 & 2.4 & 2.4 & 2.2 \\
\hline $\begin{array}{l}c_{\mathbf{R}}^{\mathbf{0}}(\mathrm{MMA}) / \\
\mathrm{mol} \cdot \mathrm{L}^{-1} \cdot 10^{-5}\end{array}$ & 1.9 & 1.8 & 1.7 & 1.1 \\
\hline $\begin{array}{c}k_{\mathrm{t}, \mathrm{SM}}^{1,1} / \\
\mathrm{L} \cdot \mathrm{mol}^{-1} \cdot \mathrm{s}^{-1} \cdot 10^{9}\end{array}$ & 2.7 & 3.9 & 5.5 & 3.9 \\
\hline$k_{\mathrm{t}, \mathrm{SM}}^{1,1} / k_{\mathrm{t}, \mathrm{SS}}^{1,1}$ & 3.5 & 5.0 & 7.1 & 5.4 \\
\hline$k_{\mathrm{t}, \mathrm{SM}}^{1,1} / k_{\mathrm{t}, \mathrm{MM}}^{1,1}$ & 3.0 & 4.3 & 6.1 & 4.6 \\
\hline
\end{tabular}

Furthermore, even if no chain length dependency of the termination is considered $\left(\alpha_{\mathrm{s}, \mathrm{SM}}=0\right), k_{\mathrm{t}, \mathrm{SM}}^{1,1}$ is three times higher than $k_{\mathrm{t}, \mathrm{MM}}^{1,1}$ and more than three times higher than $k_{\mathrm{t}, \mathrm{SS}}^{1,1}$.

The initial concentrations of styrene are higher than the experimental $c_{\mathrm{R}}^{0}($ sty- $\mathrm{d} 8)=2.03 \cdot 10^{-5} \mathrm{~mol} \cdot \mathrm{L}^{-1}$. The experimental initial concentration is obtained by the calibration procedure, and thus the errors of the calibration procedure might lead to this discrepancy. Moreover, the initial concentration of MMA is relatively high although no SP experiments were possible at the static field position of MMA. However, for parameter estimations with $c_{\mathrm{R}}^{0}(\mathrm{MMA})=0$ and $\alpha_{\mathrm{s}, \mathrm{SM}}=0.57$, a higher $k_{\mathrm{t}, \mathrm{SM}}^{1,1}=4.5 \cdot 10^{9} \mathrm{~L} \cdot \mathrm{mol}^{-1} \cdot \mathrm{s}^{-1}$ was obtained. This value is unrealistic such as the value of $k_{\mathrm{t}, \mathrm{SM}}^{1,1}=3.9 \cdot 10^{9} \mathrm{~L} \cdot \mathrm{mol}^{-1} \cdot \mathrm{s}^{-1}$ as will be shown next.

In Figure 5.18, the obtained $k_{\mathrm{t}, \mathrm{SM}}^{1,1}$ for the different $\alpha_{\mathrm{s}, \mathrm{SM}}$ are compared to $k_{\mathrm{t}}^{1,1}$ of the corresponding homopolymerization. Hence, it becomes obvious that $k_{\mathrm{t}}^{1,1}$ of cross-termination is significantly higher than $k_{\mathrm{t}}^{1,1}$ for the homopolymerizations. Moreover, the diffusion limit is shown as a cross in Figure 5.18. As can be seen, independent from $\alpha_{\mathrm{s}, \mathrm{SM}}, k_{\mathrm{t}, \mathrm{SM}}^{1,1}$ is higher 


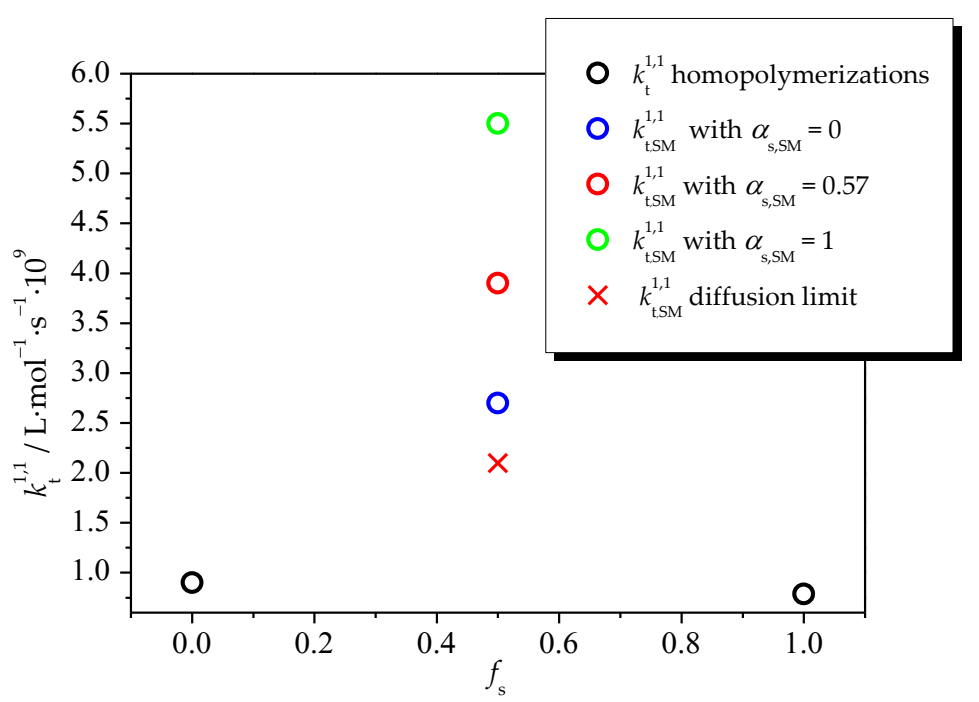

Figure 5.18: Cross-termination rate coefficients $k_{\mathrm{t}, \mathrm{SM}}^{1,1}$ determined by the parameter estimation via PREDICI ${ }^{\circledR}$ considering different $\alpha_{\mathrm{s}, \mathrm{SM}}$ for simulation variant A. For comparison, $k_{\mathrm{t}}^{1,1}$ for the homopolymerization are given, too. The cross demonstrates the diffusion limit obtained by the Smoluchowski and Stokes-Einstein equation (Equation 2.25).

than the diffusion limit. Consequently, the so-obtained $k_{\mathrm{t}, S \mathrm{M}}^{1,1}$ is physically and chemically unrealistic.

All this results in the question whether the used PREDICI ${ }^{\circledR}$ model is reliable and whether a refinement of the model can provide more realistic values of $k_{\mathrm{t}, \mathrm{SM}}^{1,1}$.

\subsubsection{Influence of Equal Termination}

Since the termination is diffusion-controlled for homopolymerizations, it should also be case for copolymerizations. Hence, the termination reaction should be independent from the terminal and penultimate units. Therefore, the termination reactions were treated equally in PREDICI $^{\circledR}$ simulations and the obtained $k_{\mathrm{t}, \mathrm{copo}}^{1,1}$ is the overall $k_{\mathrm{t}}^{1,1}$ for all termination reactions (simulation variant $\mathrm{B}$ ). The so-obtained concentration-time profiles are depicted in Figure 5.19. In comparison to 


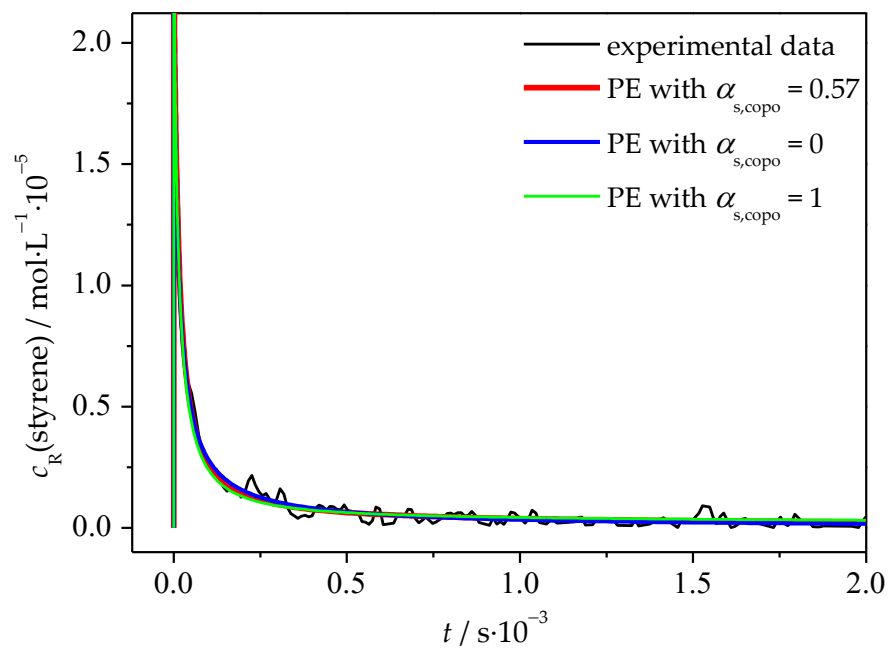

Figure 5.19: Experimental concentration-time profile for the radical copolymerization of styrene-d8 and MMA $\left(f_{\text {Sty }}=0.498\right.$ and $\left.f_{\text {MMA }}=0.502\right)$ in bulk at $333 \mathrm{~K}$. In comparison, the concentration-time profiles obtained from the parameter estimation (PE) via PREDICI ${ }^{\circledR}$ are shown considering different $\alpha_{\mathrm{s}, \text { copo }}$ for simulation variant B.

Figure 5.17, the PE with equal termination reactions provides a better description of the experimental data than simulation variant A. Moreover, only small differences for the concentration-time profiles for the different $\alpha_{\mathrm{s}, \text { copo }}$ can be observed.

The estimated parameters are given in Table 5.9. $k_{\mathrm{t}, \mathrm{copo}}^{1,1}$ is significantly lower than $k_{\mathrm{t}, S \mathrm{M}}^{1,1}$ from simulation variant A (Table 5.8). Nevertheless, $k_{\mathrm{t}, \text { copo }}^{1,1}$ is significantly higher than $k_{\mathrm{t}}^{1,1}$ for the homopolymerizations. $k_{\mathrm{t}, \text { copo }}^{1,1}$ for $\alpha_{\mathrm{s}, \text { copo }}=0.57$ is nearly two times higher than $k_{\mathrm{t}, \mathrm{SS}}^{1,1}$ and by a factor of 1.7 higher than $k_{\mathrm{t}, \mathrm{MM}}^{1,1} \quad k_{\mathrm{t}, \mathrm{copo}}^{1,1}$ is also higher than both $k_{\mathrm{t}}^{1,1}$ of the homopolymerizations if no chain length dependency of the termination is considered $\left(\alpha_{\mathrm{s}, S M}=0\right)$.

Furthermore, $c_{\mathrm{R}}^{0}(\mathrm{MMA})$ is low which is more realistic than the results for simulation variant A. Additionally, $k_{\mathrm{t}, \mathrm{copo}}^{1,1}$ is shown in Figure 5.20 in comparison to $k_{\mathrm{t}}^{1,1}$ of the homopolymerizations. As can be seen, $k_{\mathrm{t}, \mathrm{copo}}^{1,1}$ is 
Table 5.9: From the parameter estimation (simulation variant $B$ ) obtained values for $c_{\mathrm{R}}^{0}$ (styrene), $c_{\mathrm{R}}^{0}$ (MMA) and $k_{\mathrm{t}, \text { copo }}^{1,1}$ for different $\alpha_{\mathrm{s}}$. For comparison, the ratios $k_{\mathrm{t}, \mathrm{copo}}^{1,1} / k_{\mathrm{t}, \mathrm{SS}}^{1,1}$ and $k_{\mathrm{t}, \mathrm{copo}}^{1,1} / k_{\mathrm{t}, \mathrm{MM}}^{1,1}$ are given, too.

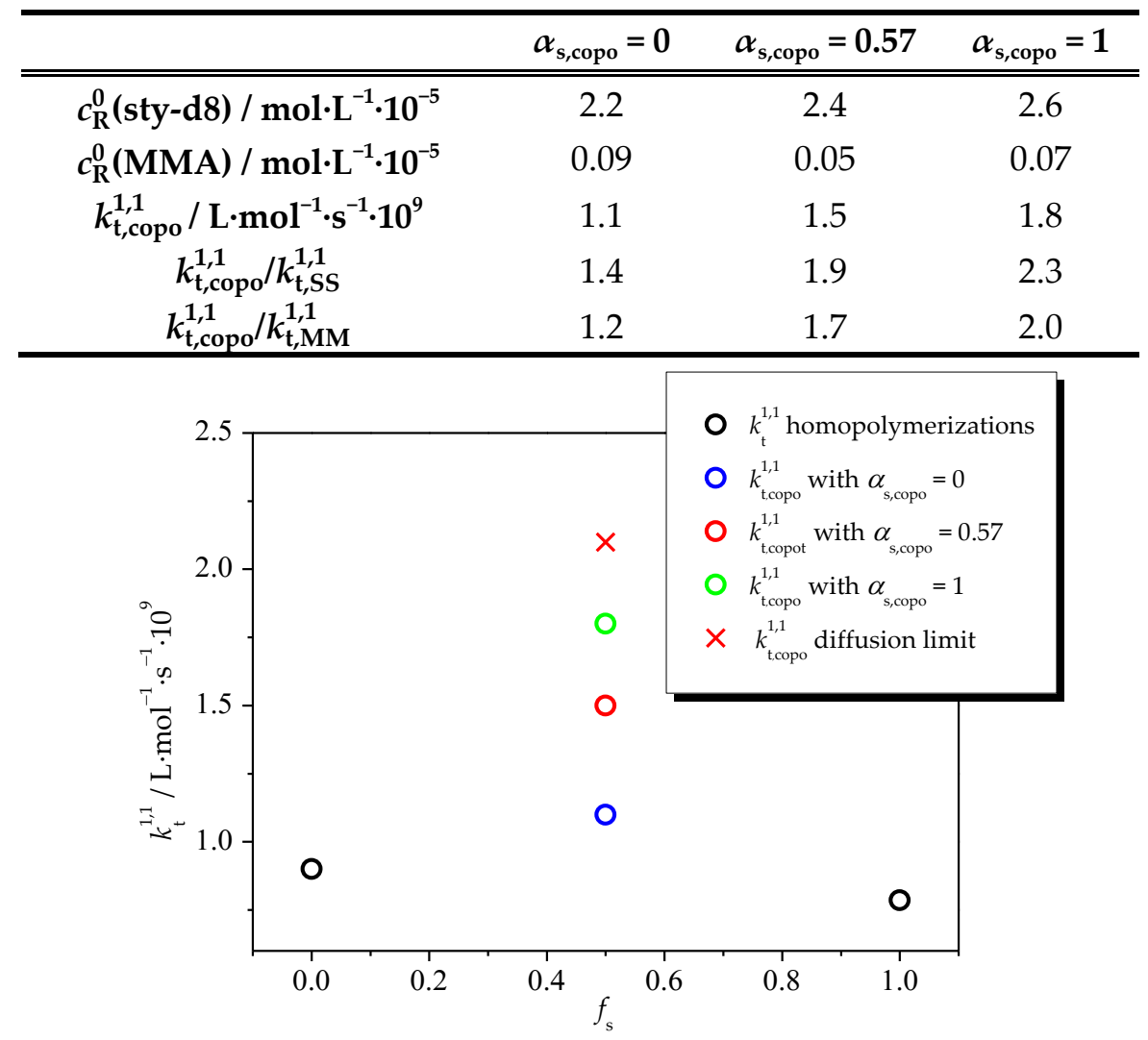

Figure 5.20: Overall termination rate coefficient $k_{\mathrm{t}, \mathrm{copo}}^{1,1}$ determined by the parameter estimation via PREDICI ${ }^{\circledR}$ considering different $\alpha_{\mathrm{s}, \text { copo }}$ for simulation variant $\mathrm{B}$. For comparison, $k_{\mathrm{t}}^{1,1}$ for the homopolymerization are given, too. The cross demonstrates the diffusion limit obtained by the Smoluchowski and Stokes-Einstein equation (Equation 2.25).

significantly higher than $k_{t}^{1,1}$ of the homopolymerizations. Furthermore, $k_{\mathrm{t}, \mathrm{copo}}^{1,1}$ is below the diffusion limit, and hence this model provides a more realistic value than simulation variant $A$. Nonetheless, some further possible reasons for such a high $k_{\mathrm{t}, \mathrm{copo}}^{1,1}$ should be analyzed. 


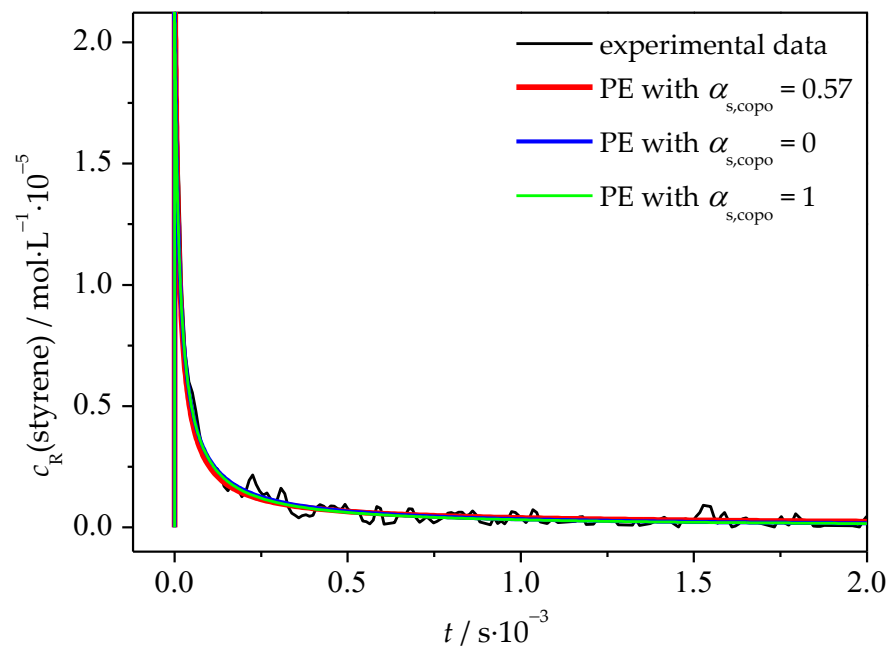

Figure 5.21: Experimental concentration-time profile for the radical copolymerization of styrene-d8 and MMA $\left(f_{\text {Sty }}=0.498\right.$ and $\left.f_{\text {MMA }}=0.502\right)$ in bulk at $333 \mathrm{~K}$. In comparison, the concentration-time profiles obtained from the parameter estimation (PE) via PREDICI ${ }^{\circledR}$ are shown considering different $\alpha_{\mathrm{s}, \text { copo }}$ for simulation variant C.

\subsubsection{Influence of Copopolymerization Parameters}

As shown in Chapter 5.2, the literature known copolymerization parameters are uncertain because they cannot describe the styrene radical fraction. Thus, the influence of the copolymerization parameters on the determination of $k_{\mathrm{t}, \mathrm{copo}}^{1,1}$ has to be analyzed. The termination reactions were treated equally like in simulation variant $B$.

Therefore, the manually obtained copolymerization parameters (Table 5.5) from this work were implemented in the PREDICI ${ }^{\circledR}$ model. The simulated concentration-time profiles agree perfectly with the experimental data and no significant differences can be observed for the different $\alpha_{\mathrm{s}, \mathrm{SM}}$ (Figure 5.21).

Regarding Table 5.10, $k_{\mathrm{t}, \text { copo }}^{1,1}$ is higher than $k_{\mathrm{t}, \text { copo }}^{1,1}$ for the homopolymerizations and higher than $k_{\mathrm{t}, \mathrm{copo}}^{1,1}$ from simulation variant $\mathrm{B}$ 
Table 5.10: From the parameter estimation (simulation variant $C$ ) obtained values for $c_{\mathrm{R}}^{0}$ (styrene), $c_{\mathrm{R}}^{0}$ (MMA) and $k_{\mathrm{t}, \mathrm{copo}}^{1,1}$ for different $\alpha_{\mathrm{s}}$. For comparison, the ratios $k_{\mathrm{t}, \text { copo }}^{1,1} / k_{\mathrm{t}, \mathrm{SS}}^{1,1}$ and $k_{\mathrm{t}, \text { copo }}^{1,1} / k_{\mathrm{t}, \mathrm{MM}}^{1,1}$ are given, too.

\begin{tabular}{|c|c|c|c|}
\hline & $\alpha_{\mathrm{s}, \mathrm{copo}}=0$ & $\alpha_{\mathrm{s}, \mathrm{copo}}=0.57$ & $\alpha_{\mathrm{s}, \mathrm{copo}}=1$ \\
\hline$c_{\mathrm{R}}^{0}$ (styrene) $/ \mathrm{mol} \cdot \mathrm{L}^{-1} \cdot 10^{-5}$ & 2.2 & 2.5 & 2.6 \\
\hline$c_{\mathrm{R}}^{0}(\mathrm{MMA}) / \mathrm{mol} \cdot \mathrm{L}^{-1} \cdot 10^{-5}$ & 0.2 & 0.1 & 0.2 \\
\hline$k_{\mathrm{t}, \mathrm{copo}}^{1,1} / \mathrm{L} \cdot \mathrm{mol}^{-1} \cdot \mathrm{s}^{-1} \cdot 10^{9}$ & 1.5 & 1.8 & 2.1 \\
\hline$k_{\mathrm{t}, \text { copo }}^{1,1} / k_{\mathrm{t}, \mathrm{SS}}^{1,1}$ & 1.9 & 2.3 & 2.7 \\
\hline$k_{\mathrm{t}, \mathrm{copo}}^{1,1} / k_{\mathrm{t}, \mathrm{MM}}^{1,1}$ & 1.7 & 2.0 & 2.3 \\
\hline
\end{tabular}

(Table 5.9). For $\alpha_{\mathrm{s}}=0.57, k_{\mathrm{t}, \mathrm{copo}}^{1,1}$ is higher by the factor of 2.3 than $k_{\mathrm{t}, \mathrm{SS}}^{1,1}$ and two times higher than $k_{\mathrm{t}, \mathrm{MM}}^{1,1}$. The obtained $c_{\mathrm{R}}^{0}(\mathrm{MMA})$ are low, and thus they are realistic.

In Figure 5.22, $k_{\mathrm{t}, \mathrm{copo}}^{1,1}$ for different $\alpha_{\mathrm{s}, \text { copo }}$ are shown. For comparison, $k_{\mathrm{t}}^{1,1}$ for both homopolymerizations are given, too. As can be seen, $k_{\mathrm{t}, \text { copo }}^{1,1}$ is significantly higher than $k_{\mathrm{t}}^{1,1}$ for both homopolymerizations. Moreover, $k_{\mathrm{t}, \text { copo }}^{1,1}$ for $\alpha_{\mathrm{s}, \text { copo }}=1$ is equal to the diffusion limit. Thus, this value is not physically and chemically realistic. However, for both homopolymerizations $\alpha_{\mathrm{s}}$ was significantly below 1 and should be also in this range for the copolymerization. For $\alpha_{\mathrm{s}}=0.57, k_{\mathrm{t}, \mathrm{copo}}^{1,1}$ is significantly below the diffusion limit, and in consequence this value is trustable. 


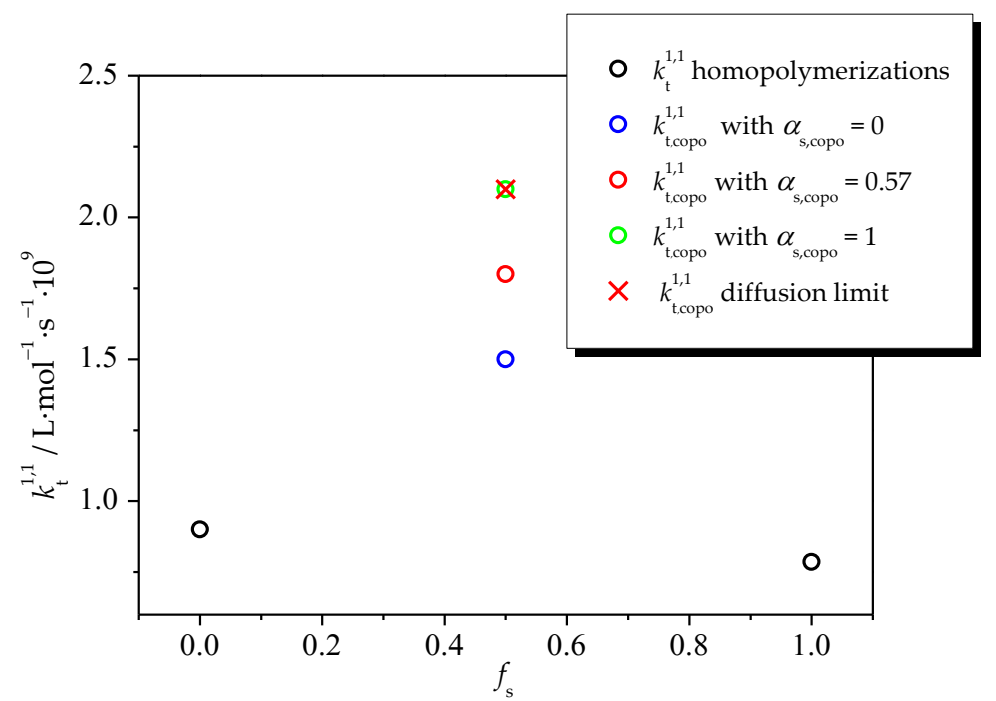

Figure 5.22: Overall termination rate coefficient $k_{\mathrm{t}, \mathrm{copo}}^{1,1}$ determined by the parameter estimation via PREDICI ${ }^{\circledR}$ considering different $\alpha_{\mathrm{s}, \text { copo }}$ for simulation variant $\mathrm{C}$. For comparison, $k_{\mathrm{t}}^{1,1}$ for the homopolymerization are given, too. The cross demonstrates the diffusion limit obtained by the Smoluchowski and Stokes-Einstein equation (Equation 2.25).

\subsubsection{Influence of Different Chain Lengths}

What has not been considered so far is that the macroradicals can have different chain lengths. To investigate the influence of varying chain length on the determination of $k_{\mathrm{t}, \text { copo }}^{1,1}$, the diffusion mean model (Equation 2.18) was implemented into the PREDICI ${ }^{\circledR}$ model. The termination reactions were treated equally (simulation variant $B$ ) and the copolymerization parameters from this work were implemented (simulation variant $\mathrm{C}$ )

The so-obtained concentration-time profiles agree with the experimental ones (Figure 5.23). As before, no significant differences for the different $\alpha_{\mathrm{s}, \text { copo }}$ can be observed. 


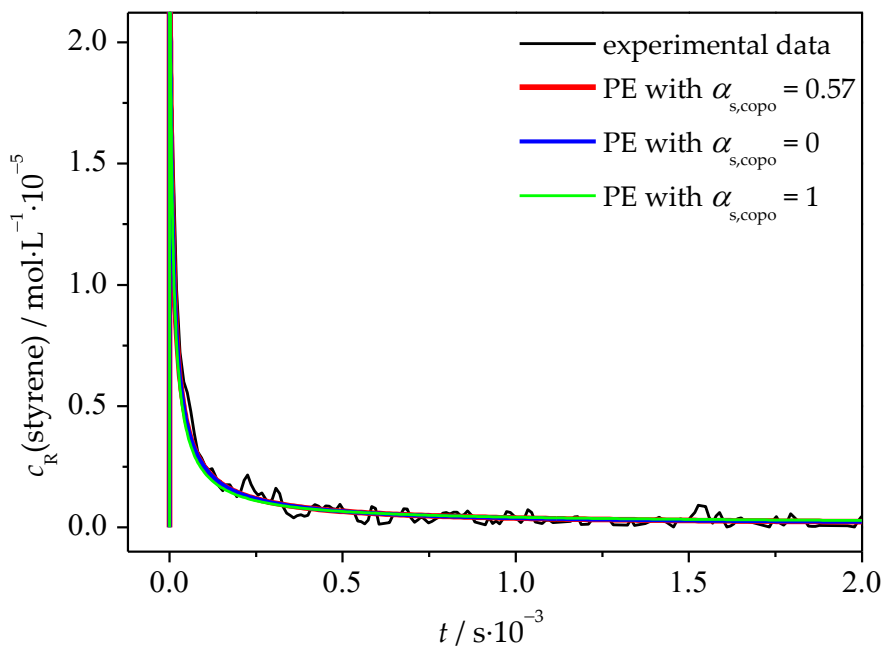

Figure 5.23: Experimental concentration-time profile for the radical copolymerization of styrene-d8 and MMA $\left(f_{\text {sty }}=0.498\right.$ and $\left.f_{\mathrm{MMA}}=0.502\right)$ in bulk at $333 \mathrm{~K}$. In comparison, the concentration-time profiles obtained from the parameter estimation (PE) via PREDICI ${ }^{\circledR}$ are shown considering different $\alpha_{\mathrm{s}, \text { copo }}$ for simulation variant $\mathrm{D}$.

Table 5.11: From the parameter estimation obtained (simulation variant $\mathrm{D}$ ) values for $c_{\mathrm{R}}^{0}$ (styrene), $c_{\mathrm{R}}^{0}(\mathrm{MMA})$ and $k_{\mathrm{t}, \mathrm{copo}}^{1,1}$ for different $\alpha_{\mathrm{s}}$. For comparison, the ratios $k_{\mathrm{t}, \mathrm{copo}}^{1,1} / k_{\mathrm{t}, \mathrm{SS}}^{1,1}$ and $k_{\mathrm{t}, \mathrm{copo}}^{1,1} / k_{\mathrm{t}, \mathrm{MM}}^{1,1}$ are given, too.

\begin{tabular}{|c|c|c|c|}
\hline & $\alpha_{\mathrm{s}, \mathrm{copo}}=0$ & $\alpha_{\mathrm{s}, \mathrm{copo}}=0.57$ & $\alpha_{\mathrm{s}, \mathrm{copo}}=1$ \\
\hline$c_{\mathrm{R}}^{0}(\mathrm{sty}-\mathrm{d} 8) / \mathrm{mol} \cdot \mathrm{L}^{-1} \cdot 10^{-5}$ & 2.2 & 2.6 & 2.9 \\
\hline$c_{\mathrm{R}}^{0}(\mathrm{MMA}) / \mathrm{mol} \cdot \mathrm{L}^{-1} \cdot 10^{-5}$ & 0.2 & 0.1 & 0.2 \\
\hline$k_{\mathrm{t}, \mathrm{copo}}^{1,1} / \mathrm{L} \cdot \mathrm{mol}^{-1} \cdot \mathrm{s}^{-1} \cdot 10^{9}$ & 1.6 & 2.1 & 2.7 \\
\hline$k_{\mathrm{t}, \mathrm{copo}}^{1,1} / k_{\mathrm{t}, \mathrm{SS}}^{1,1}$ & 2.0 & 2.7 & 3.5 \\
\hline$k_{\mathrm{t}, \mathrm{copo}}^{1,1} / k_{\mathrm{t}, \mathrm{MM}}^{1,1}$ & 1.8 & 2.3 & 3.0 \\
\hline
\end{tabular}

The results are given in Table 5.11. $k_{\mathrm{t}, \mathrm{copo}}^{1,1}$ is again significantly higher than $k_{\mathrm{t}}^{1,1}$ of the homopolymerizations. Without chain length dependency of the termination $\left(\alpha_{\mathrm{s}, \text { copo }}=0\right), k_{\mathrm{t}, \text { copo }}^{1,1}$ is almost two times higher than $k_{\mathrm{t}}^{1,1}$ 


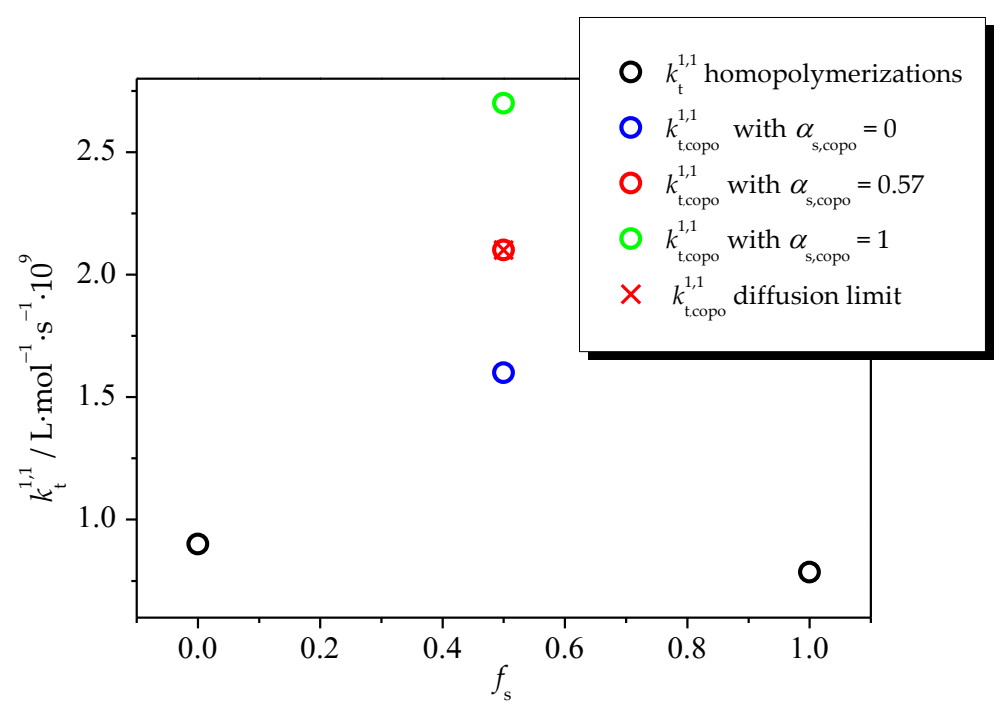

Figure 5.24: Overall termination rate coefficient $k_{\mathrm{t}, \text { copo }}^{1,1}$ determined by the parameter estimation via PREDICI ${ }^{\circledR}$ considering different $\alpha_{\mathrm{s}, \text { copo }}$ for simulation variant D. For comparison, $k_{\mathrm{t}}^{1,1}$ for the homopolymerization are given, too. The cross demonstrates the diffusion limit obtained by the Smoluchowski and Stokes-Einstein equation (Equation 2.25).

of the homopolymerizations. Moreover, $k_{\mathrm{t}, \text { copo }}^{1,1}$ for $\alpha_{\mathrm{s}, \text { copo }}=0.57$ has the same value as $k_{\mathrm{t}, \mathrm{copo}}^{1,1}$ for $\alpha_{\mathrm{s}, \text { copo }}=1$ obtained from simulation variant $\mathrm{C}$.

In comparison to simulation variant $\mathrm{C}, k_{\mathrm{t}, \text { copo }}^{1,1}$ for $\alpha_{\mathrm{s}, \text { copo }}=1$ of the last simulation has the same value as the diffusion limit, and thus it is not reliable. This can be also observed for $k_{\mathrm{t}, \mathrm{copo}}^{1,1}$ for $\alpha_{\mathrm{s}, \mathrm{copo}}=0.57$ of simulation variant $\mathrm{D}$ (Figure 5.24). Thus, $\alpha_{\mathrm{s}, \text { copo }}$ might be smaller than $\alpha_{\mathrm{s}, \text { copo }}=0.57$ and is perhaps more in the region of styrene $\left(\alpha_{\mathrm{s}}=0.51\right)$.

Finally, all simulation variants lead to significantly higher $k_{\mathrm{t}, \mathrm{SM}}^{1,1}$ and $k_{\mathrm{t}, \mathrm{copo}}^{1,1} \quad$ respectively, than $k_{\mathrm{t}}^{1,1}$ for the corresponding homopolymerizations. Since simulation variant A provides values of $k_{\mathrm{t}, \mathrm{SM}}^{1,1}$ clearly above the diffusion limit, these results will not be regarded further. The results from the other simulation variants are concluded in Table 5.12. 
Table 5.12: Conclusion of the results from the different simulation variants (simulation variant $\mathrm{A}$ excluded) at $333 \mathrm{~K}$. In comparison, the literature values at $298 \mathrm{~K}$ are given, too.

\begin{tabular}{|c|c|c|c|c|}
\hline & $\begin{array}{c}\text { simulation } \\
\text { variant B } \\
\left(\alpha_{\mathrm{s}, \text { copo }}=0.57\right)\end{array}$ & $\begin{array}{c}\text { simulation } \\
\text { variant } C \\
\left(\alpha_{\mathrm{s}, \text { copo }}=0.57\right)\end{array}$ & $\begin{array}{c}\text { simulation } \\
\text { variant } D \\
\left(\alpha_{\mathrm{s}, \mathrm{copo}}=0.57\right)\end{array}$ & $\begin{array}{c}\text { literature } \\
\text { values at } \\
298 K^{[53]}\end{array}$ \\
\hline $\begin{array}{c}k_{\mathrm{t}, \mathrm{SS}}^{1,1} / \\
\mathrm{L} \cdot \mathrm{mol}^{-1} \cdot \mathrm{s}^{-1} \cdot 10^{8}\end{array}$ & 7.8 & 7.8 & 7.8 & $1.1 \pm 0.2$ \\
\hline $\begin{array}{c}k_{\mathrm{t}, \mathrm{MM}}^{1,1} / \\
\mathrm{L} \cdot \mathrm{mol}^{-1} \cdot \mathrm{s}^{-1} \cdot 10^{8}\end{array}$ & 9.0 & 9.0 & 9.0 & $1.5 \pm 0.3$ \\
\hline $\begin{array}{c}k_{\mathrm{t}, \mathrm{copo}}^{1,1} / \\
\mathrm{L} \cdot \mathrm{mol}^{-1} \cdot \mathrm{s}^{-1} \cdot 10^{8}\end{array}$ & $15 \pm 1$ & $18 \pm 1$ & $21 \pm 1$ & $2.2 \pm 0.5$ \\
\hline$k_{\mathrm{t}, \mathrm{copo}}^{1,1} / k_{\mathrm{t}, \mathrm{SS}}^{1,1}$ & 1.9 & 2.3 & 2.7 & 2.0 \\
\hline$k_{\mathrm{t}, \mathrm{copo}}^{1,1} / k_{\mathrm{t}, \mathrm{MM}}^{1,1}$ & 1.7 & 2.0 & 2.3 & 1.5 \\
\hline
\end{tabular}

Furthermore, the results for $k_{\mathrm{t}, \mathrm{copo}}^{1,1}$ from Olaj et al. for the copolymerization of styrene and MMA in bulk at $298 \mathrm{~K}$ are given in Table 5.12 , too. ${ }^{[53]}$ In this previous work, $k_{\mathrm{t}, \text { copo }}^{1,1}$ was determined by extrapolation of experimental data obtained from stationary experiments. However, even in this study, $k_{t, \text { copo }}^{1,1}$ was significantly higher than $k_{t}^{1,1}$ of the homopolymerizations determined by the same method (Figure 5.25). A direct comparison of the experimental values and the literature values is not possible due to the different temperatures and different used methods. Therefore, the ratios $k_{\mathrm{t}, \mathrm{copo}}^{1,1} / k_{\mathrm{t}, \mathrm{SS}}^{1,1}$ and $k_{\mathrm{t}, \mathrm{copo}}^{1,1} / k_{\mathrm{t}, \mathrm{MM}}^{1,1}$ are compared. The best agreement with the literature is obtained by simulation variant B. Here, the literature known copolymerization parameters were used. Nonetheless, as shown in Chapter 5.2, the literature known copolymerization parameters are not reliable. Therefore, the simulation variant $C$ should be preferred over simulation variant $B$. Whether simulation variant $D$ should be preferred over variant $C$ is not easy to answer. Therefore, further investigations are required. 


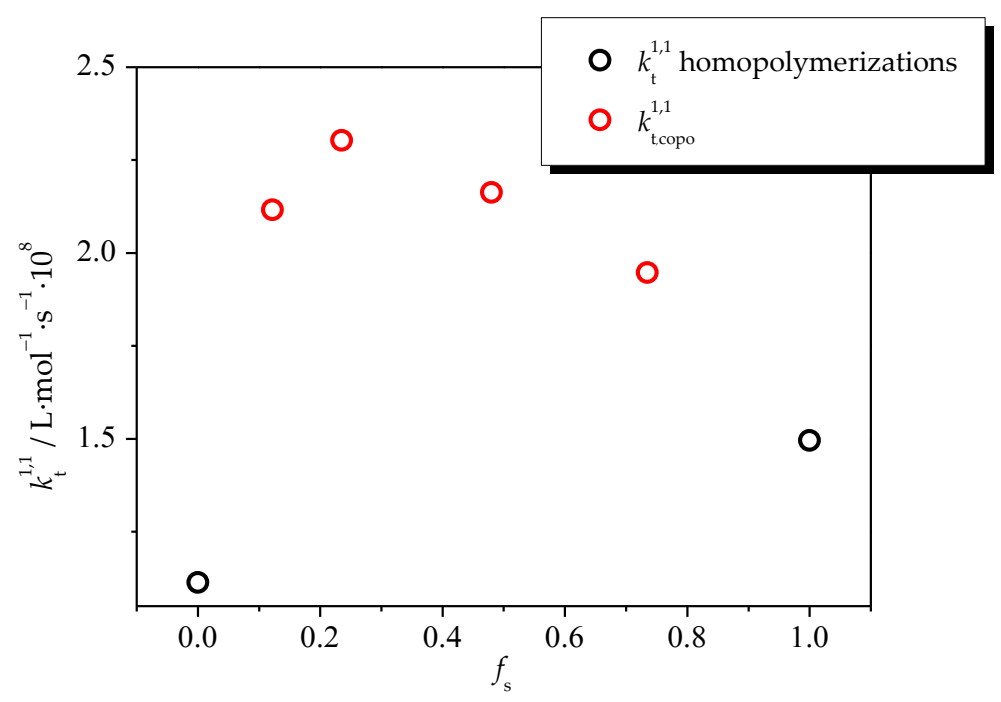

Figure 5.25: Literature values of the overall termination rate coefficient $k_{\mathrm{t}, \mathrm{copo}}^{1,1}$ for the copolymerization of styrene and MMA in bulk at $298 \mathrm{~K} .^{[53]}$

However, it is remarkable that $k_{\mathrm{t}, \text { copo }}^{1,1}$ is in both studies higher than $k_{\mathrm{t}}^{1,1}$ of the homopolymerizations. $k_{t}^{1,1}$ represents the termination rate coefficient of two monomeric radicals, and thus it would be expected that $k_{\mathrm{t}, \text { copo }}^{1,1}$ is the average value both two $k_{\mathrm{t}}^{1,1}$ for the homopolymerizations. As has be shown for homopolymerizations, $k_{\mathrm{t}}^{1,1}$ can be correlated to the ratio of $R_{\mathrm{c}}$ and $r_{1}$ (Equation 4.4). Thus, $k_{\mathrm{t}}^{1,1}$ is higher if $R_{\mathrm{c}}$ is higher or $r_{1}$ is smaller than for the homopolymerizations. Of course, $r_{1}$ is the hydrodynamic radius of the monomers, and hence it should not be different to those in the homopolymerizations. Nonetheless, it was argued that $k_{\mathrm{t}}^{1,1}$ could be influenced by the properties of the macroradicals. Also Olaj et al. ${ }^{[53]}$ tried to explain this high $k_{\mathrm{t}, \text { copo }}^{1,1}$ by the chain flexibility of the macroradicals(Equation 2.43). They argued that the chain flexibility is improved if the penultimate or penpenultimate unit differs from the terminal model. In contrast, Dynamic Light Scattering (DLS) measurements for determining $r_{\mathrm{h}}$ for a random copolymer of styrene and MMA have shown that $r_{\mathrm{h}}$ for the copolymer is in between the $r_{\mathrm{h}}$ of the homopolymers in a good solvent such as THF. ${ }^{[133]}$ Hence, it would be interesting whether similar results would be obtained for the 
copolymer in bulk. Moreover, further investigations into other copolymerization systems could provide insight whether the styreneMMA copolymerization is an exception or not.

\subsection{Conclusion and Further Perspectives}

Within this chapter, EPR spectra during the radical copolymerization of styrene-d8 and MMA were measured for different compositions. In comparison to the spectra measured during the homopolymerization of both monomers, a clear assignment of the different signals in the copolymerization spectra was possible. Moreover, the copolymerization spectrum with the lowest styrene feed fraction $\mathrm{d} 8\left(f_{\text {sty-d } 8}=0.118\right.$ and $f_{\mathrm{MMA}}=0.881$ ) agrees perfectly with the superposition of the corresponding homopolymerization spectra. However, for composition $\left(f_{\text {sty-d } 8}=0.467\right.$ and $f_{\mathrm{MMA}}=0.533$ ) near to the azeotropic point only the styrene-d8 signal was observable which can be explained by the high cross-propagation rate $k_{\mathrm{p}, \mathrm{MS}}$.

Furthermore, with MATLAB ${ }^{\circledR}$ simulations it was possible to obtain the $a_{\mathrm{hf}}$ of the copolymerization spectra. Since the styrene- $\mathrm{d} 8$ spectrum is a singlet, the $a_{\text {hf }}$ of MMA were interesting. For the copolymerization spectra with the lowest styrene feed fraction $\left(f_{\text {sty-d } 8}=0.118\right.$ and $\left.f_{\mathrm{MMA}}=0.881\right)$, small differences of $a_{\mathrm{hf}}$ for the copolymerization spectrum were found compared to the $a_{\mathrm{hf}}$ of the homopolymerization spectrum of MMA. These small differences can be caused by the penultimate unit effect. Moreover, with this MATLAB ${ }^{\circledR}$ simulations, it was possible to determine the radical fraction of styrene-d8 during the copolymerization for different compositions. The radical fraction of styrene was always higher than the feed fraction of styrene. Additionally, the radical fraction of styrene-d8 agrees perfectly with the values from a study of Heuts et al.$^{[112]}$ It could be shown that it is impossible to describe the radical fraction of styrene for different styrene feed fractions by the penultimate model with the literature known copolymerization parameters. However, if the copolymerization parameters were determined by a least-squares fit of the experimental data of the radical styrene fraction of this work, the obtained copolymerization parameters could not describe the literature 
values for $k_{\mathrm{p}, \mathrm{copo}}$ and $F_{\mathrm{s}}$. Therefore, all copolymerization parameters were manually adjusted in Excel ${ }^{\circledR}$, and thus it could be shown that it is possible to describe all experimental data from this work and literature with the implicit and explicit model, respectively. The errors for the so-obtained copolymerization parameters were also determined manually. Thus, it could be shown that by a simultaneous fitting of all experimental data, more precise copolymerization parameters can be obtained. Furthermore, investigations into other copolymerizations systems could be performed to determine the radical fraction. Thus, it could be investigated whether one radical fraction is higher than its feed fraction. For instance, EPR investigation into the copolymerization of styrene- $\mathrm{d} 5$ and cyclohexyl methacrylate- $\mathrm{d} 5$ in benzene at $60^{\circ} \mathrm{C}$ also showed a higher fraction of macroradicals with styrene-d5 as terminal unit. ${ }^{[114]}$ Here, the EPR signals were double integrated to calculate the radical fraction. ${ }^{[114]}$ However, for the method used within this thesis no double integration is required, and thus the error might be smaller. A good candidate for further investigations is the copolymerization of styrene and DMA. An advantage of this copolymerization is that DMA is less polar than MMA, and thus this copolymerization can be measured in bigger sample tubes. Hence, the signal-to-noise ratio can be improved due to the higher sample volume, and hence the evaluation of the spectra becomes easier. Additionally, the copolymerization of styrene and maleic anhydride is interesting for further investigations because it is an alternating copolymerization system.

Moreover, the SP experiments from a previous work of the author was reevaluated again. Since a transfer step occurs during a copolymerization, it is not possible to evaluate the SP data as for homopolymerizations. Hence, the concentration-time profiles had to be evaluated via PREDICI ${ }^{\circledR}$. Compared to the previous work of the author, the implicit penultimate model was implemented into the PREDICI ${ }^{\circledR}$ model instead of the terminal model. Furthermore, several aspects were regarded in different simulation variants. With the different models, a parameter estimation was performed to determined $k_{\mathrm{t}, \mathrm{SM}}^{1,1}$ and $k_{\mathrm{t}, \mathrm{copo}}^{1,1}$, respectively, and the initial concentrations of both monomers were estimated, too. In simulation variant $\mathrm{A}$, it was assumed that the homo-terminations differ 
from the cross-termination. Therefore, for the homo-terminations, composite parameters of the corresponding homopolymerization were taken from literature. Because the viscosity of the copolymerization differs from the viscosity of the homopolymerization, the viscosity of the copolymerization was considered in simulation variant $A$. This resulted in a high cross-termination rate coefficient $k_{\mathrm{t}, \mathrm{SM}}^{1,1}$ which was above the diffusion limit. Hence, this value is chemically and physically unrealistic. However, since the termination is diffusion controlled, the terminal and penultimate units of the macroradicals should not have such a great impact on the termination kinetics. Thus, all termination reaction were treated equally, and an overall termination rate coefficient $k_{\mathrm{t}, \text { copo }}^{1,1}$ was determined (simulation variant $\mathrm{B}$ ). From the parameter estimation, $k_{\mathrm{t}, \text { copo }}^{1,1}$ was determined to be significantly higher than $k_{\mathrm{t}}^{1,1}$ for the homopolymerization. Nonetheless, $k_{\mathrm{t}, \mathrm{copo}}^{1,1}$ was below the diffusion limit and is more reliable than the result from simulation variant $\mathrm{A}$. The radical fraction of styrene has shown that the literature known copolymerization parameters are not reliable. Hence, the copolymerization parameters obtained by manually fitting were taken for further simulations (simulation variant C). The so-obtained $k_{\mathrm{t}, \text { copo }}^{1,1}$ was significantly higher than $k_{t}^{1,1}$ for the homopolymerization but was also below the diffusion limit. In simulation variant $D$, different chain length of the macroradicals were regarded. The estimated $k_{\mathrm{t}, \text { copo }}^{1,1}$ was again higher than $k_{\mathrm{t}}^{1,1}$ for the homopolymerization. Nevertheless, $k_{\mathrm{t}, \mathrm{copo}}^{1,1}$ was equal to the diffusion limit, and thus this result is not reliable. Perhaps, $\alpha_{\mathrm{s}, \text { copo }}$ is smaller than the expected $\alpha_{\mathrm{s}, \text { copo }}=0.57$. However, comparing the results from simulation variant B-D to a previous study of Olaj et al., ${ }^{[53]} k_{\mathrm{t}, \text { copo }}^{1,1}$ from this work are confirmed. Although the previous study was performed at another temperature and via a stationary method, similar ratios $k_{\mathrm{t}, \mathrm{Copo}}^{1,1} / k_{\mathrm{t}, \mathrm{SS}}^{1,1}$ and $k_{\mathrm{t}, \mathrm{copo}}^{1,1} / k_{\mathrm{t}, \mathrm{MM}}^{1,1}$ as in this work were obtained. The reason behind this high $k_{\mathrm{t}, \mathrm{copo}}^{1,1}$ is not clear. Olaj et al. argued that the chain flexibility of the copolymeric macroradicals differs compared to the homopolymeric macroradicals. ${ }^{[53]}$ The flexibility is increased if the penultimate unit differs from the terminal unit. Since $k_{\mathrm{t}}^{1,1}$ can be correlated to ratio of $R_{\mathrm{c}}$ and $r_{1}$, it might be that $R_{\mathrm{c}}$ is higher or $r_{1}$ is lower than for the homopolymerization. However, $r_{\mathrm{h}}$ determined for a random copolymer of styrene and MMA 
determined via DLS measurements was in-between $r_{\mathrm{h}}$ of the homopolymers in a good solvent such as THF. ${ }^{[133]}$ In consequence, it would be interesting whether similar results would be found in bulk.

Moreover, further investigations are necessary to clearly answer the open questions. For further information about the termination kinetics of copolymerizations, SP experiments for further compositions of the styrene-MMA copolymerization are interesting. Moreover, from measurements at different temperatures, the Arrhenius parameters might be obtained. Thus, it can be analyzed whether the activation energy of $k_{\mathrm{t}, \mathrm{copo}}^{1,1}$ can be correlated to the activation energy of the intrinsic viscosity.

Furthermore, it would be interesting whether similar results are obtained for other copolymerizations such as styrene and DMA. Since the propagation and termination kinetics of the homopolymerization of methacrylates shows a family behavior, it is interesting whether similar tendencies are obtained for copolymerization of styrene with other methacrylates. The benefits of the copolymerization of styrene and DMA due to SP-PLP-EPR measurements are given above.

With further refinements of the PREDICI ${ }^{\circledR}$ simulations, more precise values of $k_{\mathrm{t}, \text { copo }}^{1,1}$ can be estimated. Implementing the explicit model is challenging but might be possible. Moreover, due to the chain-length below $i<10$, the chain-length dependent propagation can be used. However, to obtain exact knowledge about the chain-length dependent propagation of a copolymerization is difficult because it is also difficult for homopolymerization (Chapter 2.4.1).

Finally, although some questions are still left open, new insights into the termination kinetics and into the copolymerization parameters were obtained within this work. Hence, the obtained results in this chapter bring the investigation into the kinetics of copolymerization a step forward and open new perspectives for further investigations. 


\section{CLOSING REMARKS}

During this work some important aspects of the chain-length dependent termination kinetics of radical homo- and copolymerizations were intensively investigated by applying SP-PLP-EPR.

For the first time, investigations into the chain-length dependent termination kinetics of the PnMA radical homopolymerization in bulk were performed. It was shown that this radical homopolymerization is perfectly represented by the composite model. The composite exponents $\alpha_{\mathrm{s}}=0.56 \pm 0.08$ and $\alpha_{1}=0.16 \pm 0.04$ for PnMA agree with the exponents of other methacrylates and monomers and with the theoretically predicted values. Moreover, the activation energy for the termination rate coefficient of two monomeric radicals $E_{\mathrm{A}}\left(k_{\mathrm{t}}^{1,1}\right)=12 \mathrm{~kJ} \cdot \mathrm{mol}^{-1}$ is similar to the activation energy of the fluidity $E_{\mathrm{A}}\left(\eta^{-1}\right)$. Thus, $k_{\mathrm{t}}^{1,1} \cdot \eta$ is constant at different temperatures. By comparing the obtained results with other methacrylates, it could be demonstrated that $k_{\mathrm{t}}^{1,1} \cdot \eta$ decreases with increasing size of ester side chain due to the increasing hydrodynamic radius.

Furthermore, for 2-EHMA and DMA, a significant temperature dependency was observed for $i_{\mathrm{c}}$. Here, $i_{\mathrm{c}}$ shows a sigmoidal behavior and decreases with increasing temperature. Such a temperature dependency of $i_{\mathrm{c}}$ was not obtained for PnMA $\left(i_{\mathrm{c}}=67 \pm 10\right)$. Hence, the size of the ester side chain influences the temperature dependency of $i_{\mathrm{c}}$. This can be underlined by the determined inflection point of the sigmoidal curves for 2-EHMA and DMA which is $305 \mathrm{~K}$ for 2-EHMA and $319 \mathrm{~K}$ for DMA, respectively. For future studies it would be interesting to investigate other methacrylates such as hexyl methacrylate (HMA) in bulk. Moreover, it would be interesting whether a similar temperature dependency of $i_{\mathrm{c}}$ can be observed for methacrylates in solution. For a more detailed discussion of future research perspectives the reader is referred to Chapter 4.4 . 
Additionally, important parts of the experimental setup were replaced or repaired. Hence, with the investigations into the termination kinetics of the methacrylates within this work, these changes could be validated.

In the last part of this thesis, the kinetics of the radical copolymerization of styrene and MMA were thoroughly investigated. Therefore, EPR spectra for different copolymerization compositions were measured. For simplification, fully deuterated styrene was used. A clear assignment of the signals to the two possible terminal units was obtained by comparing the copolymerization spectra with the corresponding homopolymerization spectra. Furthermore, via Matlab ${ }^{\circledR}$ simulations the styrene radical fractions for the different copolymerization compositions were determined directly from the EPR spectra. However, this radical styrene fractions for different feed fractions of styrene cannot be described by the penultimate model with the literature known copolymerization parameters. Therefore, the radical styrene fraction and literature known values for the propagation rate coefficient and the copolymer composition of the copolymerization of styrene and MMA were manually fitted by the implicit and the explicit model. Hence, more precise copolymerization parameters were obtained for both models. Moreover, single pulse experiments were reevaluated via a refined PREDICI ${ }^{\circledR}$ model. This model is based on the implicit model and $k_{\mathrm{t}, \text { cross }}^{1,1}$ and $k_{\mathrm{t}, \mathrm{copo}}^{1,1}$, respectively, were determined by parameter estimation. Several influences on the termination kinetics such as the viscosity, equal termination reactions, different copolymerization parameters and different chain lengths of the macroradicals were investigated in different simulation variants. In simulation variant $\mathrm{A}$, the two different homoterminations and one cross-termination were implemented. Therefore, the influence of the viscosity on the homo-terminations was considered. However, $k_{\mathrm{t} \text {,cross }}^{1,1}$ was significantly above the diffusion limit, and thus it is unrealistic. In consequence, the termination reactions were treated equally in simulation variant $\mathrm{B}$ to determine $k_{\mathrm{t}, \mathrm{copo}}^{1,1}$. The more precise copolymerization parameters obtained in this work were included in simulation variant $C$ and the different chain lengths of macroradicals in simulation variant $D$. In simulation variant $B$ and $C, k_{t, \text { copo }}^{1,1}$ was significantly higher than $k_{\mathrm{t}}^{1,1}$ for the relating homopolymerization. 
Nonetheless, in both simulation variants $k_{\mathrm{t}, \mathrm{copo}}^{1,1}$ was clearly below the diffusion limit, and hence these values are realistic. For simulation variant $\mathrm{D}, k_{\mathrm{t}, \mathrm{copo}}^{1,1}$ was equal to the diffusion limit, and thus further refinements are required. However, all simulation variants led to a significant higher $k_{\mathrm{t}, \text { copo }}^{1,1}$ than $k_{\mathrm{t}}^{1,1}$ for the corresponding homopolymerization. This might be caused by a higher chain flexibility of the copolymeric macroradicals compared to the homopolymeric macroradicals. For a deeper understanding, more investigations into the kinetics of radical copolymerizations are required. For the styrene-MMA copolymerization, SP experiments of additional compositions and temperatures could be performed. Moreover, with kinetic studies of the styrene-DMA copolymerization it could be analyzed whether the radical fraction of styrene is also higher than the feed fraction of styrene and whether a higher $k_{t, \text { copo }}^{1,1}$ is obtained, too. The alternating copolymerization of styrene and maleic anhydride would be a suitable candidate for further investigations. By implementing the explicit penultimate model into PREDICI ${ }^{\circledR}$, a refinement of the model might be achieved, and hence more precise $k_{\mathrm{t}, \mathrm{copo}}^{1,1}$ could be estimated. All these concluded future perspectives are also described in Chapter 5.5 in more detail. To sum up, SP-PLP-EPR was successfully applied for the first time to investigate the radical copolymerization in more detail than before. Thus, new perspectives for further investigations into the kinetics of radical copolymerizations were created.

Beside the high interest into the kinetics of radical copolymerization, investigation into the kinetics of radical homopolymerizations in aqueous solution are of great importance. With SP-PLP-EPR, the propagation of those systems can be determined which cannot be determined as usually via PLP-SEC ${ }^{[47]}$ Investigations into the kinetics of sodium acrylate would be interesting. Hence, the applications of SP-PLP-EPR are wide and far from exhausted. 



\section{APPENDIX}

\section{ABBREVIATIONS}

A

$A_{0}$

$a, b$

$a_{\mathrm{hf}}$

$\alpha$

$\alpha_{1}$

$\alpha_{\mathrm{s}}$

$A_{\mathrm{SM}}$

ATRP

BA

BMA

tert-BMA

$C_{1}$

$c_{\mathrm{DCP}}$

$c_{\mathrm{I}^{*}}$

$c_{\mathrm{I}}$

$c_{\mathrm{M}}$

$c_{\mathrm{MMMP}}$

$c_{\mathrm{R}}$

$c_{\mathrm{R}}^{0}$

$\mathrm{CR}$

$C_{\mathrm{RD}}$

CRP proportional constant for Heller-McConnell

equation

pre-exponential factor

type of monomer

hyperfine coupling constant

power-law exponent for chain-length dependent termination rate coefficient

power-law exponent for long chain region

power-law exponent for short chain region

ratio of macroradicals with styrene as terminal unit

and those with MMA as terminal unit

butyl acrylate

butyl methacrylate

tert-butyl methacrylate

concentration of DCP

concentration of primary radicals

concentration of initiator

concentration of monomer

concentration of MMMP

concentration of (marco)radicals

initial radical concentration

chemical reaction

reaction diffusion constant

conventional radical polymerization 


\begin{tabular}{|c|c|}
\hline$c_{R}(\mathrm{TEMPO})$ & radical concentration of TEMPO \\
\hline CTA & chain-transfer agent \\
\hline$C_{\mathrm{tr}, \mathrm{X}}$ & transfer constant \\
\hline CW & continues wave \\
\hline$c_{\mathrm{X}}$ & concentration of transfer agent $X$ \\
\hline DCP & dicumyl peroxide \\
\hline$D^{i}$ & individual diffusion coefficient \\
\hline DMA & dodecyl methacrylate \\
\hline $\mathrm{dmm}$ & diffusion mean model \\
\hline$E_{\mathrm{A}}$ & activation energy \\
\hline 2-EHMA & 2-ethylhexyl methacrylate \\
\hline EPR & electron paramagnetic resonance \\
\hline$\eta$ & viscosity \\
\hline$\eta_{0}$ & viscosity at $0 \%$ conversion \\
\hline $\begin{array}{l}\eta_{\mathrm{r}} \\
\text { et al. }\end{array}$ & relative viscosity \\
\hline$f$ & efficiency of initiator \\
\hline$f_{a}$ & feed fraction of monomer $a$ in a copolymerization \\
\hline$F_{a}$ & amount of monomer $a$ in copolymer \\
\hline gmm & geometric mean model \\
\hline$h_{1}, h_{2}$ & calibration constants \\
\hline HMA & hexyl methacrylate \\
\hline $\mathrm{hmm}$ & harmonic mean model \\
\hline$I$ & EPR signal intensity \\
\hline$I_{0}$ & initial EPR signal intensity \\
\hline $\mathrm{I}^{\bullet}$ & primary radical \\
\hline $\mathrm{I}_{2}$ & initiator \\
\hline$i, j$ & chain length of macroradical \\
\hline$i_{1 / 2}$ & "half-life" of first order kinetics \\
\hline$i_{\mathrm{c}}$ & crossover chain length \\
\hline
\end{tabular}


$i_{\mathrm{c}, \max }$

$i_{\mathrm{c} \text {, min }}$

$k_{\mathrm{B}}$

$k_{\mathrm{d}}$

$k_{\mathrm{D}}$

$k_{\mathrm{i}}$

$k_{\mathrm{p}}$

$k_{\mathrm{p}}^{0}$

$k_{\mathrm{p}}^{1}$

$k_{\mathrm{p}, a a}$

$k_{\mathrm{p}, a b}$

$k_{\mathrm{p}, a b d}$

$k_{\mathrm{p}, \mathrm{d}}^{0}$

$k_{\mathrm{p}, \mathrm{d}}$

$k_{\mathrm{p}, \text { copo }}$

$k_{\mathrm{p}}^{i}$

$k_{\mathrm{p}}^{\infty}$

$k_{\mathrm{p}, X}$

$k_{\mathrm{p}, \mathrm{X}}$

$\left\langle k_{\mathrm{t}}\right\rangle$

$k_{\mathrm{t}}^{0}$

$k_{\mathrm{t}}^{1,1}$

$k_{\mathrm{t}, \mathrm{copo}}^{1,1}$

$\left\langle k_{\mathrm{t}, \mathrm{CR}}\right\rangle$

upper limit of crossover chain length

lower limit of crossover chain length

Boltzmann constant

rate coefficient of the initiator decay

rate coefficient of the diffusion of two reactants

rate coefficient of the initiation

propagation rate coefficient

propagation rate coefficient without diffusion

initial propagation rate coefficient

homo-propagation rate coefficient in the terminal

model

cross-propagation rate coefficient in the terminal

model

propagation rate coefficient in the penultimate

model

propagation rate coefficient with diffusion at $0 \%$

conversion

propagation rate coefficient with diffusion

overall propagation rate coefficient of a

copolymerization

chain-length dependent propagation rate coefficient

long-chain propagation rate coefficient

diffusion-controlled propagation rate coefficient

propagation rate coefficient of transfer agent $X$

chain length averaged termination rate coefficient

termination rate coefficient of two hypothetical

coiled monomeric radicals

termination rate coefficient of two monomeric

radicals

copolymerization termination rate coefficient of two

monomeric radicals

termination rate coefficient of chemical reaction 


\begin{tabular}{|c|c|}
\hline$k_{\mathrm{t}, \text { cross }}^{1,1}$ & $\begin{array}{l}\text { cross-termination rate coefficient of two monomeric } \\
\text { radicals }\end{array}$ \\
\hline$\left\langle k_{\mathrm{t}, a a}\right\rangle$ & homo-termination rate coefficient of two \\
\hline & macroradicals with monomer $a$ as terminal unit \\
\hline$\left\langle k_{\mathrm{t}, a b}\right\rangle$ & $\begin{array}{l}\text { cross-termination rate coefficient with different } \\
\text { monomers } a \text { and } b \text { as terminal unit }\end{array}$ \\
\hline$\left\langle k_{\mathrm{t}, \mathrm{copo}}\right\rangle$ & overall termination rate coefficient in a \\
\hline & copolymerization \\
\hline$\left\langle k_{\mathrm{t}, \mathrm{d}}\right\rangle$ & termination rate coefficient with diffusion \\
\hline$k_{\mathrm{td}}$ & termination rate coefficient of disproportion \\
\hline$k_{\mathrm{t}}^{i, i}$ & $\begin{array}{l}\text { termination rate coefficient of two macroradicals } \\
\text { with the chain length } i\end{array}$ \\
\hline$k_{\mathrm{tk}}$ & termination rate coefficient of combination \\
\hline$k_{\mathrm{tr}}$ & rate coefficient of transfer \\
\hline$\left\langle k_{\mathrm{t}, \mathrm{RD}}\right\rangle$ & termination rate coefficient of reaction diffusion \\
\hline$\left\langle k_{\mathrm{t}, \mathrm{SD}}\right\rangle$ & termination rate coefficient of segmental diffusion \\
\hline$\left\langle k_{\mathrm{t}, \mathrm{TD}}\right\rangle$ & termination rate coefficient of translational diffusion \\
\hline$\left\langle k_{\mathrm{t}, \mathrm{TD}}^{0}\right\rangle$ & $\begin{array}{l}\text { termination rate coefficient of translational diffusion } \\
\text { at } 0 \% \text { conversion }\end{array}$ \\
\hline M & monomer \\
\hline$M$ & molar mass \\
\hline$m$ & mass \\
\hline MA & methyl acrylate \\
\hline MCR & mid chain radical \\
\hline MMA & methyl methacrylate \\
\hline MMMP & $\begin{array}{l}\text { 2-methyl-4(methylthio)-2-morpholino- } \\
\text { propiophenone }\end{array}$ \\
\hline$N_{\mathrm{A}}$ & Avogadro constant \\
\hline$n_{a}$ & amount of monomer $a$ \\
\hline NIR & near-infrared \\
\hline $\mathrm{P}_{1}^{\bullet}$ & monomeric radical \\
\hline$P_{a}$ & $\begin{array}{l}\text { the relative fraction of the macroradicals with } \\
\text { monomer } a \text { as terminal unit }\end{array}$ \\
\hline
\end{tabular}




\begin{tabular}{|c|c|}
\hline$P_{a b}$ & $\begin{array}{l}\text { the relative fraction of the macroradicals with } \\
\text { monomer } a \text { as penultimate unit and monomer } b \text { as } \\
\text { terminal unit }\end{array}$ \\
\hline PET & polyethylene \\
\hline $\mathrm{P}_{i}^{\bullet}$ & macroradical with chain length $i$ \\
\hline $\mathrm{P}_{i}^{\bar{E}}$ & unsaturated polymer with chain length $i$ \\
\hline $\mathrm{P}_{j}^{\mathrm{H}}$ & saturated polymer with chain length $j$ \\
\hline $\mathrm{P}_{i+j}$ & polymer with $i+j$ monomers \\
\hline PLP & pulsed laser polymerization \\
\hline PMMA & poly(methyl methacrylate) (Plexiglas ${ }^{\circledR}$ ) \\
\hline PnMA & $n$-pentyl methacrylate \\
\hline$P_{\text {Spin }}$ & spin factor \\
\hline p.r.r. & pulse repetition rate \\
\hline PUE & penultimate unit effect \\
\hline$\Phi$ & cross-termination factor \\
\hline$\Phi_{\mathrm{s}}$ & radical fraction of styrene \\
\hline$\varphi$ & quantity for chain flexibility \\
\hline$R$ & ideal gas constant \\
\hline $\begin{array}{l}r_{a}, r_{a}{ }^{\prime} \\
\text { RAFT }\end{array}$ & reactivity ratios of monomer $a$ \\
\hline$R_{\mathrm{c}}$ & capture radius \\
\hline $\mathrm{RD}$ & reaction diffusion \\
\hline $\begin{array}{l}\text { PEGMA } \\
R_{\mathrm{p}}\end{array}$ & $\begin{array}{l}\text { poly(ethylene glycol) methyl ether methacrylate } \\
\text { overall polymerization rate }\end{array}$ \\
\hline$r_{i}$ & $\begin{array}{l}\text { hydrodynamic radii of a monomer/macroradical } \\
\text { with chain length } i\end{array}$ \\
\hline$s_{a}$ & radical reactivity ratios \\
\hline SD & segmental diffusion \\
\hline SEC & size-exclusion chromatography \\
\hline SP & single pulse \\
\hline SPR & secondary propagating radical \\
\hline sty & styrene \\
\hline sty-d8 & fully deuterated styrene \\
\hline
\end{tabular}




$\begin{array}{ll}t & \text { time } \\ T & \text { temperature in } \mathrm{K} \\ t_{\mathrm{c}} & \text { crossover time } \\ \mathrm{TD} & \text { translation diffusion } \\ \text { TEMPO } & (2,2,6,6-\text { tetramethylpiperidine-1-yl)oxyl } \\ T_{\mathrm{i}} & \text { inflection point of sigmoidal fit } \\ t_{\mathrm{p}} & \text { propagation time } \\ X & \\ \mathrm{X} & \text { conversion } \\ & \text { transfer molecule }\end{array}$




\section{Figures AND TABLES}

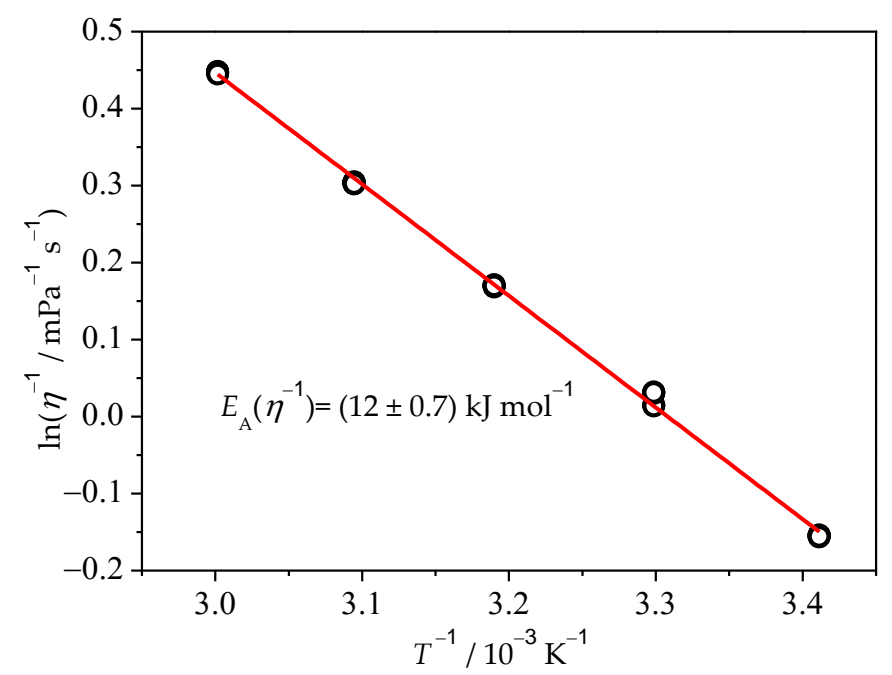

Figure II.1: Arrhenius plot of the fluidity and the determined activation energy $E_{\mathrm{A}}$ for PnMA in bulk. 


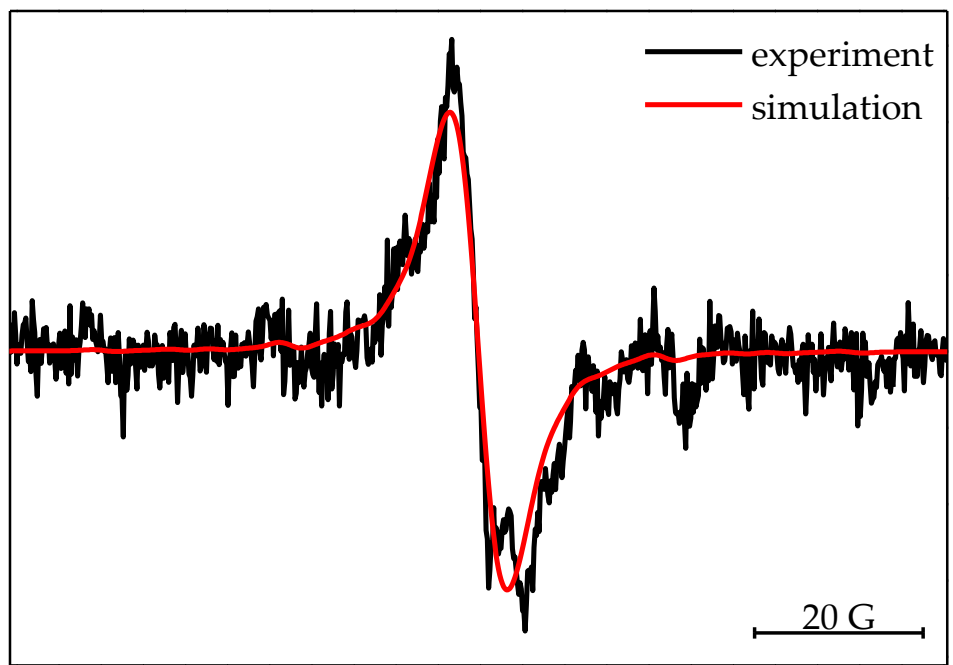

Figure II.2: EPR spectrum recorded during a radical copolymerization of styrened8 and MMA $\left(f_{\text {sty-d8 }}=0.239\right.$ and $\left.f_{\text {MMA }}=0.761\right)$ in bulk at $333 \mathrm{~K}$. The radicals were produced under continuous irradiation with a UV-lamp. The so-obtained styrene fraction is $\Phi_{\mathrm{s}}=0.91 \pm 0.04$.

Table II.1: Hyperfine coupling constants for the spectrum recorded during the radical copolymerization of styrene-d8 and MMA $\left(f_{\text {sty-d8 }}=0.239\right.$ and $\left.f_{\text {MMA }}=0.761\right)$ at $333 \mathrm{~K}$.

\begin{tabular}{ccc}
\hline & Conformer A & Conformer B \\
\hline \hline $\begin{array}{c}\text { Amount and position } \\
\text { of hydrogen }\end{array}$ & $a_{\mathrm{hf}} / \mathrm{G}$ & $a_{\mathrm{hf}} / \mathrm{G}$ \\
$\mathbf{1} \mathbf{H}_{\beta, 1}$ & 14.2 & 23.4 \\
$\mathbf{1} \mathbf{H}_{\beta, 2}$ & 8.7 & 0.2 \\
$\mathbf{3} \mathbf{H}_{\text {methyl }}$ & & 21.7 \\
\hline
\end{tabular}


II Figures and Tables

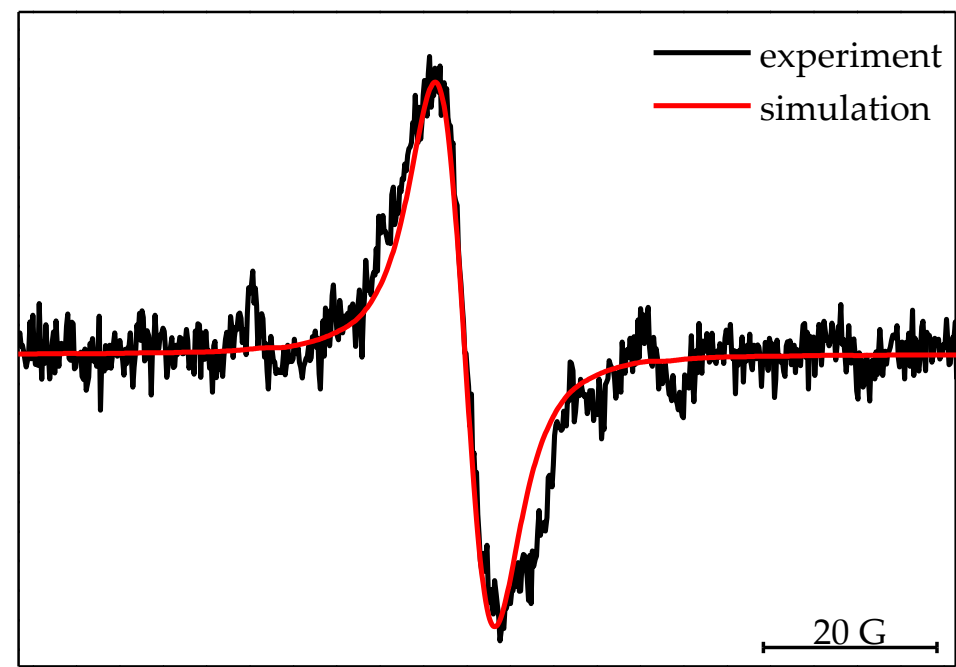

Figure II.3:

Table II.2: Hyperfine coupling constants for the spectrum recorded during the radical copolymerization of styrene- $\mathrm{d} 8$ and MMA $\left(f_{\text {sty-d } 8}=0.349\right.$ and $\left.f_{\mathrm{MMA}}=0.651\right)$ at $333 \mathrm{~K}$.

\section{Conformer A Conformer B}

\begin{tabular}{ccc}
\hline \hline $\begin{array}{c}\text { Amount and position } \\
\text { of hydrogen }\end{array}$ & $a_{\mathrm{hf}} / \mathrm{G}$ & $a_{\mathrm{hf}} / \mathrm{G}$ \\
$\mathbf{1} \mathbf{H}_{\beta, 1}$ & 14.2 & 23.4 \\
$\mathbf{1} \mathbf{H}_{\beta, 2}$ & 8.7 & 0.2 \\
$\mathbf{3} \mathbf{H}_{\text {methyl }}$ & & 22.2 \\
\hline
\end{tabular}




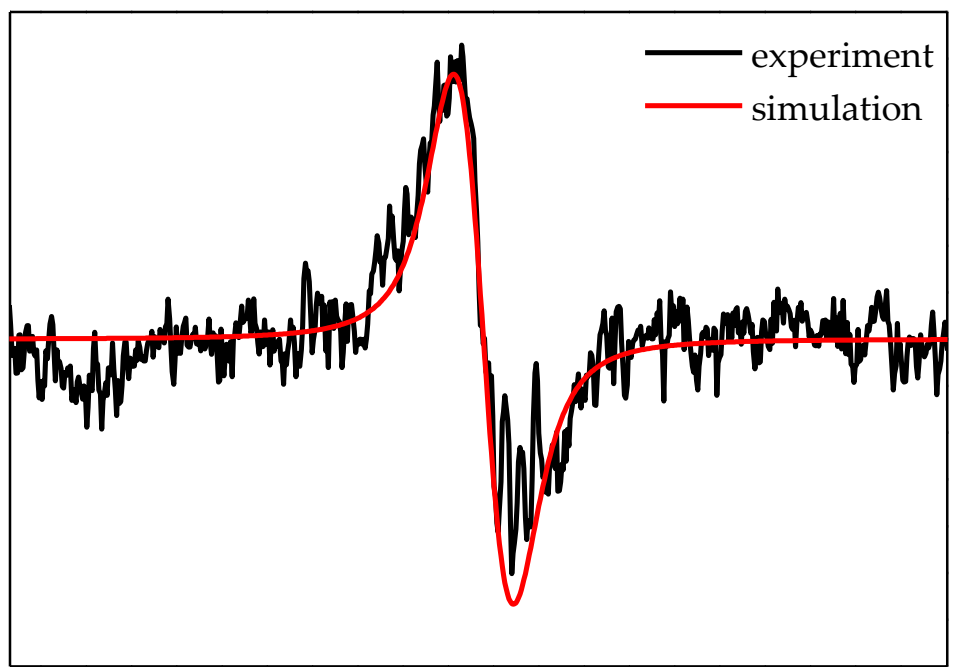

Figure II.4: EPR spectrum recorded during a radical copolymerization of styrened8 and MMA $\left(f_{\text {sty-d8 }}=0.467\right.$ and $\left.f_{\text {MMA }}=0.533\right)$ in bulk at $333 \mathrm{~K}$. The radicals were produced under continuous irradiation with a UV-lamp. The so-obtained styrene fraction is $\Phi_{\mathrm{s}}=0.95 \pm 0.04$.

Table II.3: Hyperfine coupling constants for the spectrum recorded during the radical copolymerization of styrene-d 8 and MMA $\left(f_{\text {sty-d8 }}=0.467\right.$ and $\left.f_{\mathrm{MMA}}=0.533\right)$ at $333 \mathrm{~K}$.

\section{Conformer A Conformer B}

\begin{tabular}{|c|c|c|}
\hline $\begin{array}{c}\text { Amount and position } \\
\text { of hydrogen }\end{array}$ & $a_{\mathrm{hf}} / \mathrm{G}$ & $a_{\mathrm{hf}} / \mathrm{G}$ \\
\hline $1 \mathrm{H}_{\beta, 1}$ & 14.2 & 23.4 \\
\hline $1 \mathbf{H}_{\beta, 2}$ & 8.7 & 0.2 \\
\hline $3 \mathrm{H}_{\text {methyl }}$ & \multicolumn{2}{|c|}{22.2} \\
\hline
\end{tabular}



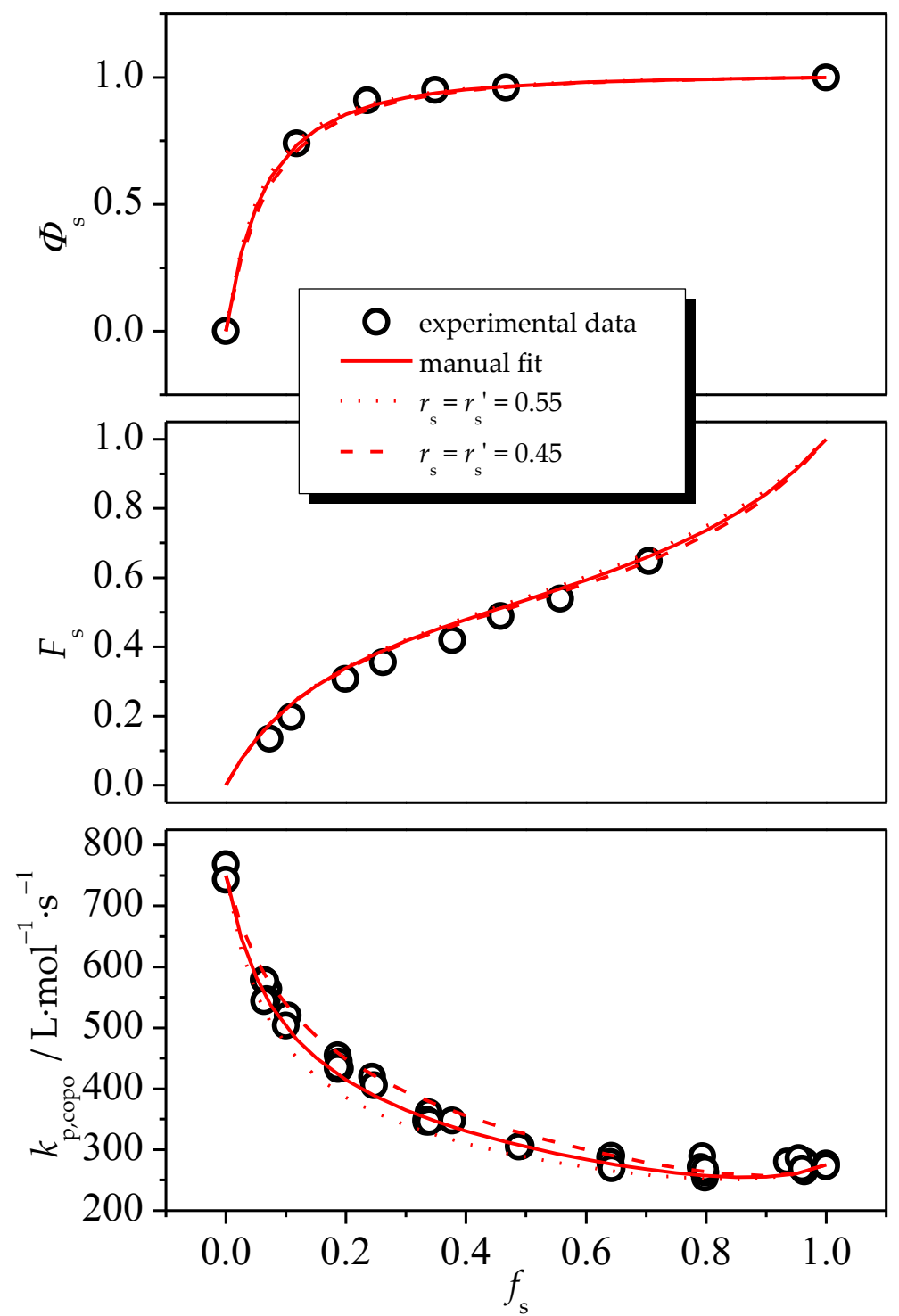

Figure II.5: Error estimation for the manually fitted copolymerization parameter $r_{\mathrm{S}}$ by the implicit penultimate model is shown as dotted and dashed red lines, respectively. The manual fit with the copolymerization parameters given in Table 5.5 is shown as solid red line. $F_{\mathrm{S}}^{[50]}$ and the overall $k_{\mathrm{p}, \text { copo }}{ }^{[131]}$ were taken from literature. 

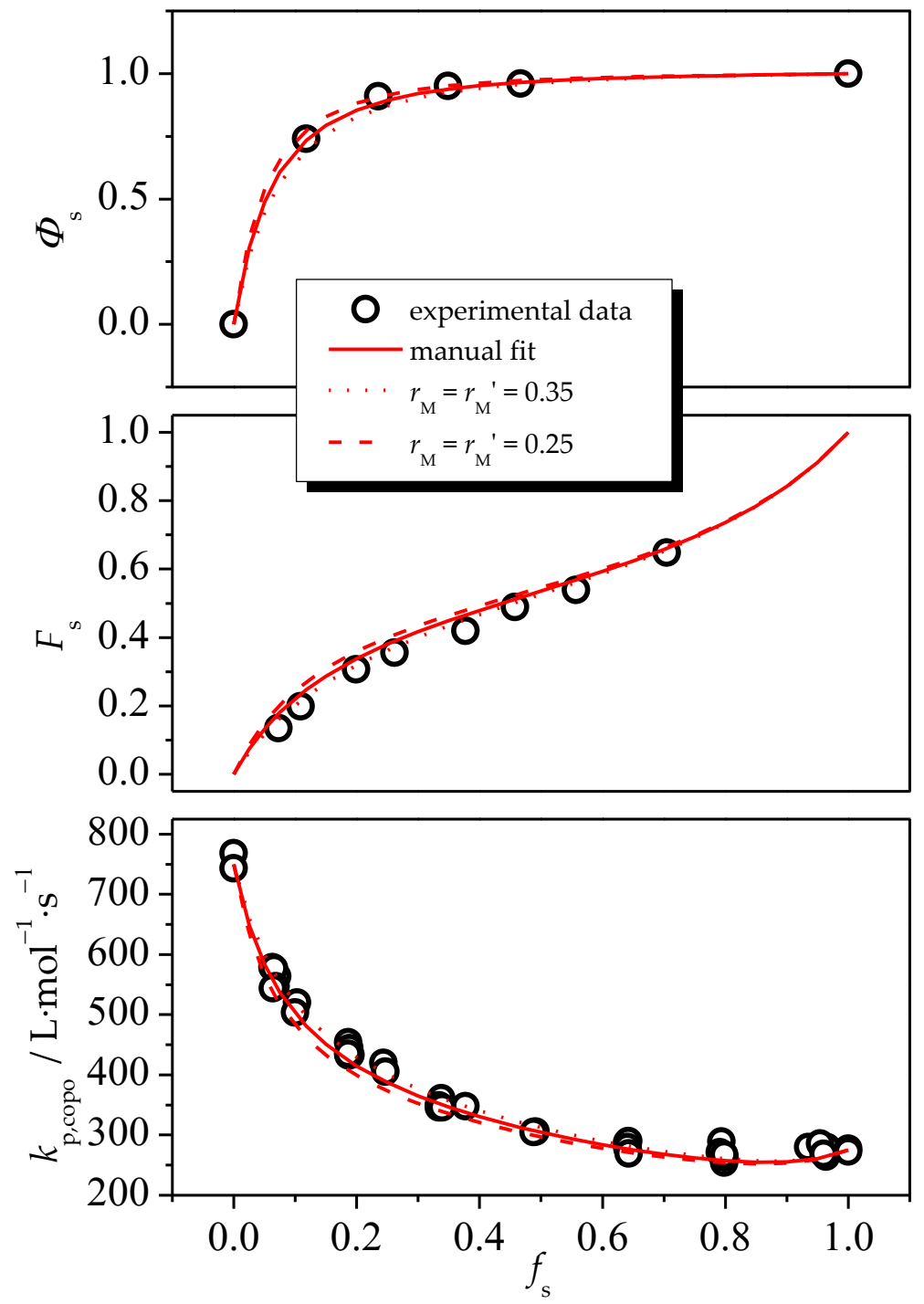

Figure II.6: Error estimation for the manually fitted copolymerization parameter $r_{\mathrm{M}}$ by the implicit penultimate model is shown as dotted and dashed red lines, respectively. The manual fit with the copolymerization parameters given in Table 5.5 is shown as solid red line. $F_{\mathrm{S}}^{[50]}$ and the overall $k_{\mathrm{p}, \text { copo }}{ }^{[131]}$ were taken from literature. 


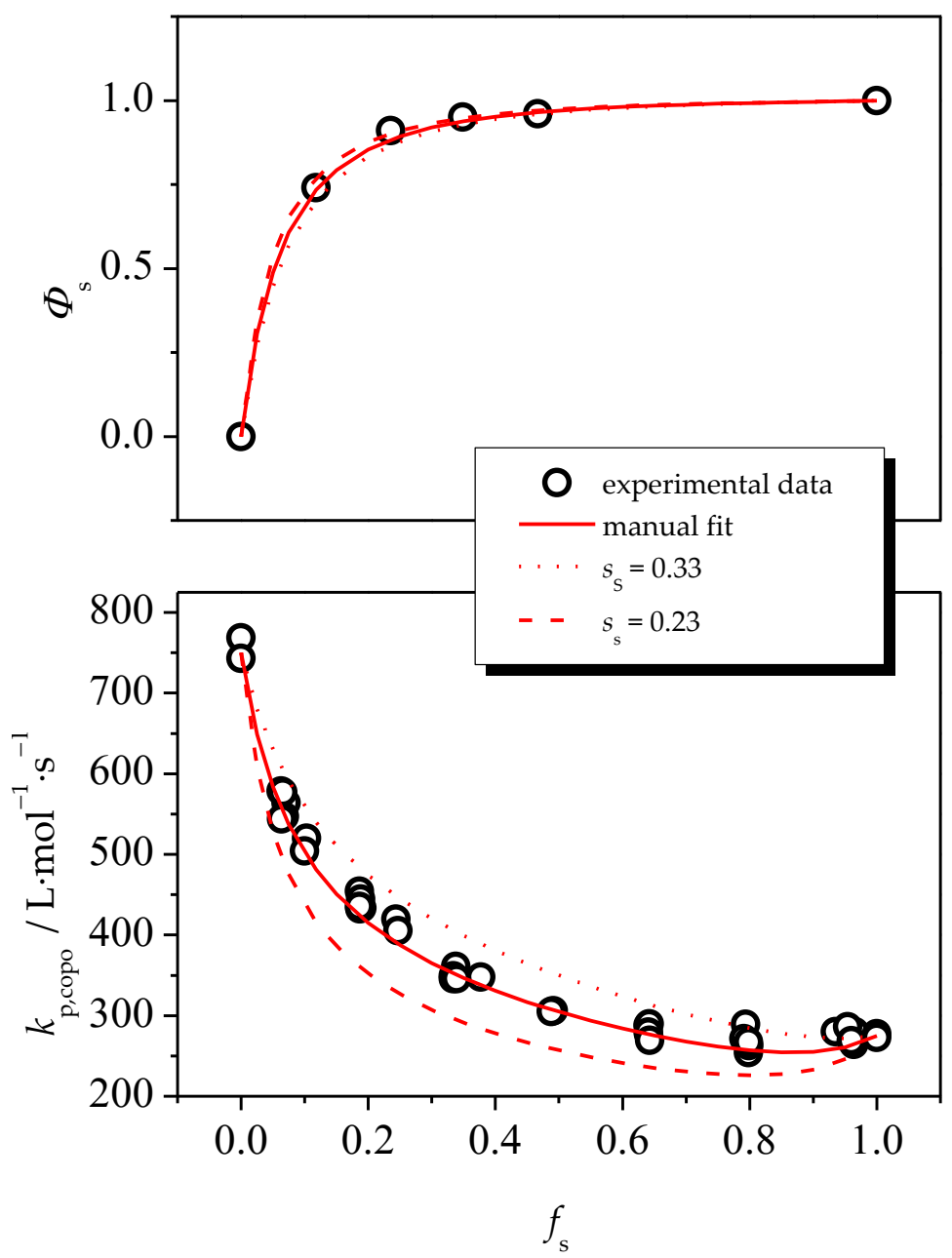

Figure II.7: Error estimation for the manually fitted copolymerization parameter $s_{\mathrm{S}}$ by the implicit penultimate model is shown as dotted and dashed red lines, respectively. The manual fit with the copolymerization parameters given in Table 5.5 is shown as solid red line. The overall $k_{\mathrm{p}, \text { copo }}{ }^{[131]}$ was taken from literature. 


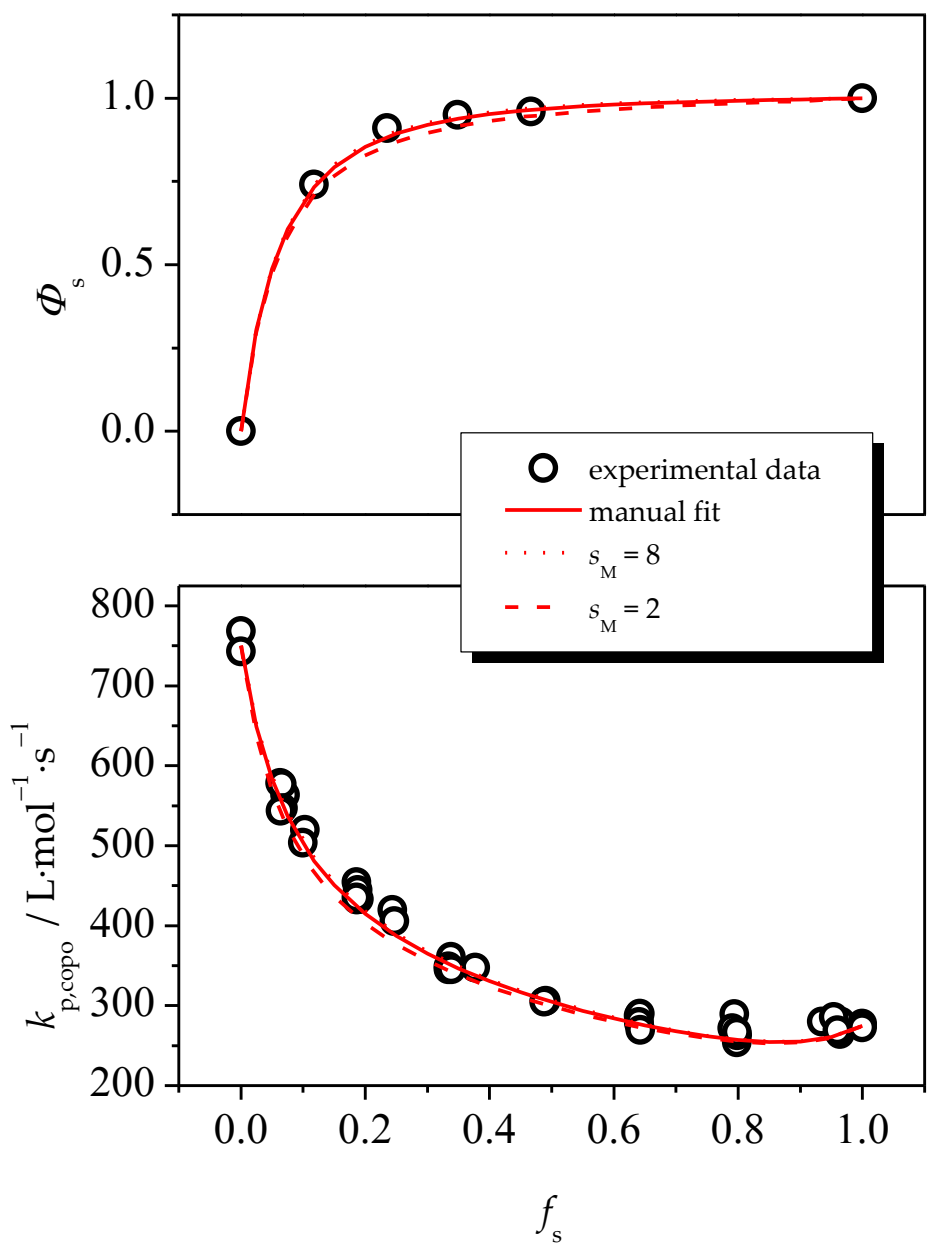

Figure II.8: Error estimation for the manually fitted copolymerization parameter $s_{\mathrm{M}}$ by the implicit penultimate model is shown as dotted and dashed red lines, respectively. The manual fit with the copolymerization parameters given in Table 5.5 is shown as solid red line. The overall $k_{\mathrm{p}, \text { copo }}{ }^{[131]}$ was taken from literature. 

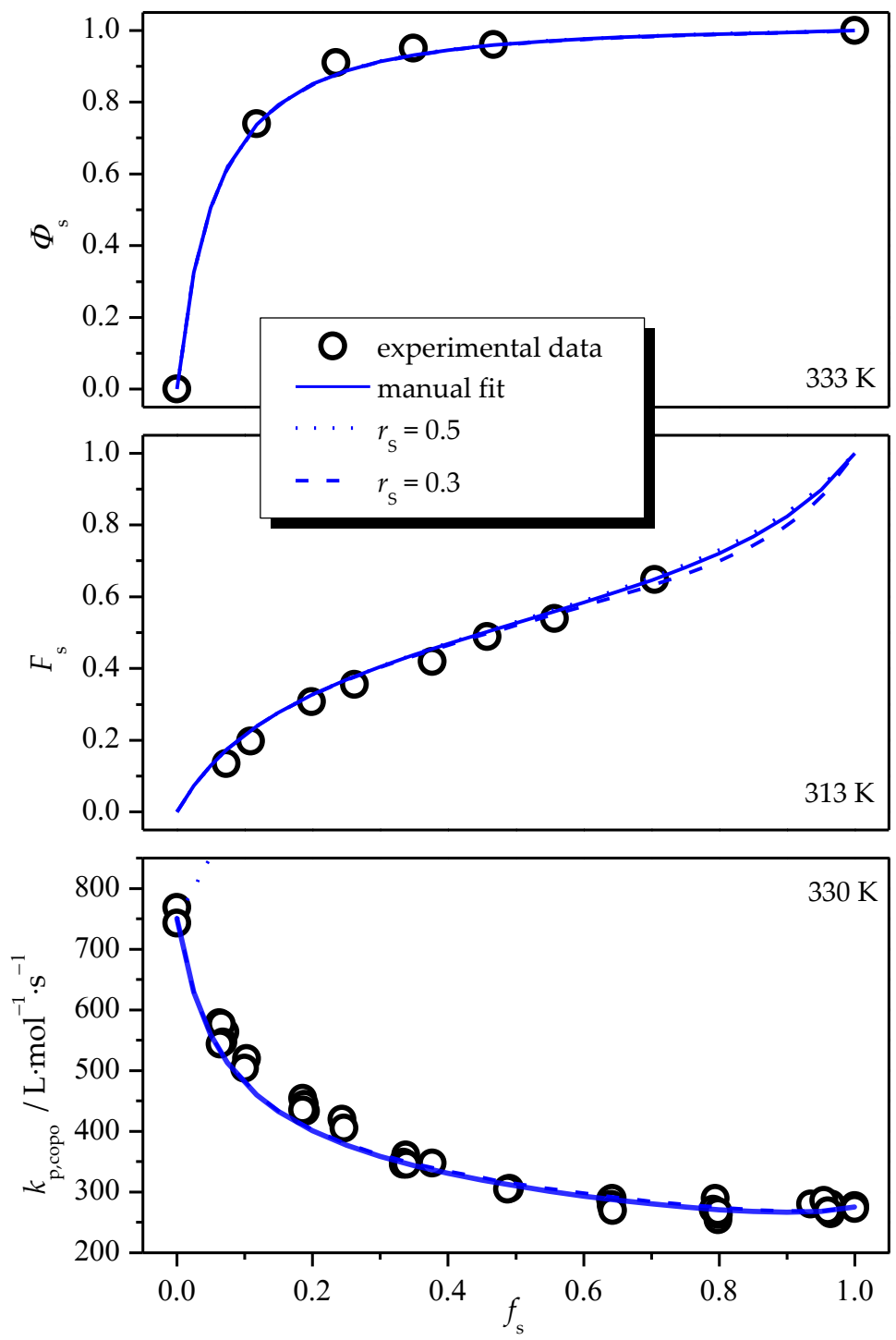

Figure II.9: Error estimation for the manually fitted copolymerization parameter $r_{\mathrm{S}}$ by the explicit penultimate model is shown as dotted and dashed blue lines, respectively. The manual fit with the copolymerization parameters given in Table 5.5 is shown as solid blue line. $F_{\mathrm{S}}^{[50]}$ and the overall $k_{\mathrm{p}, \text { copo }}{ }^{[131]}$ were taken from literature. 

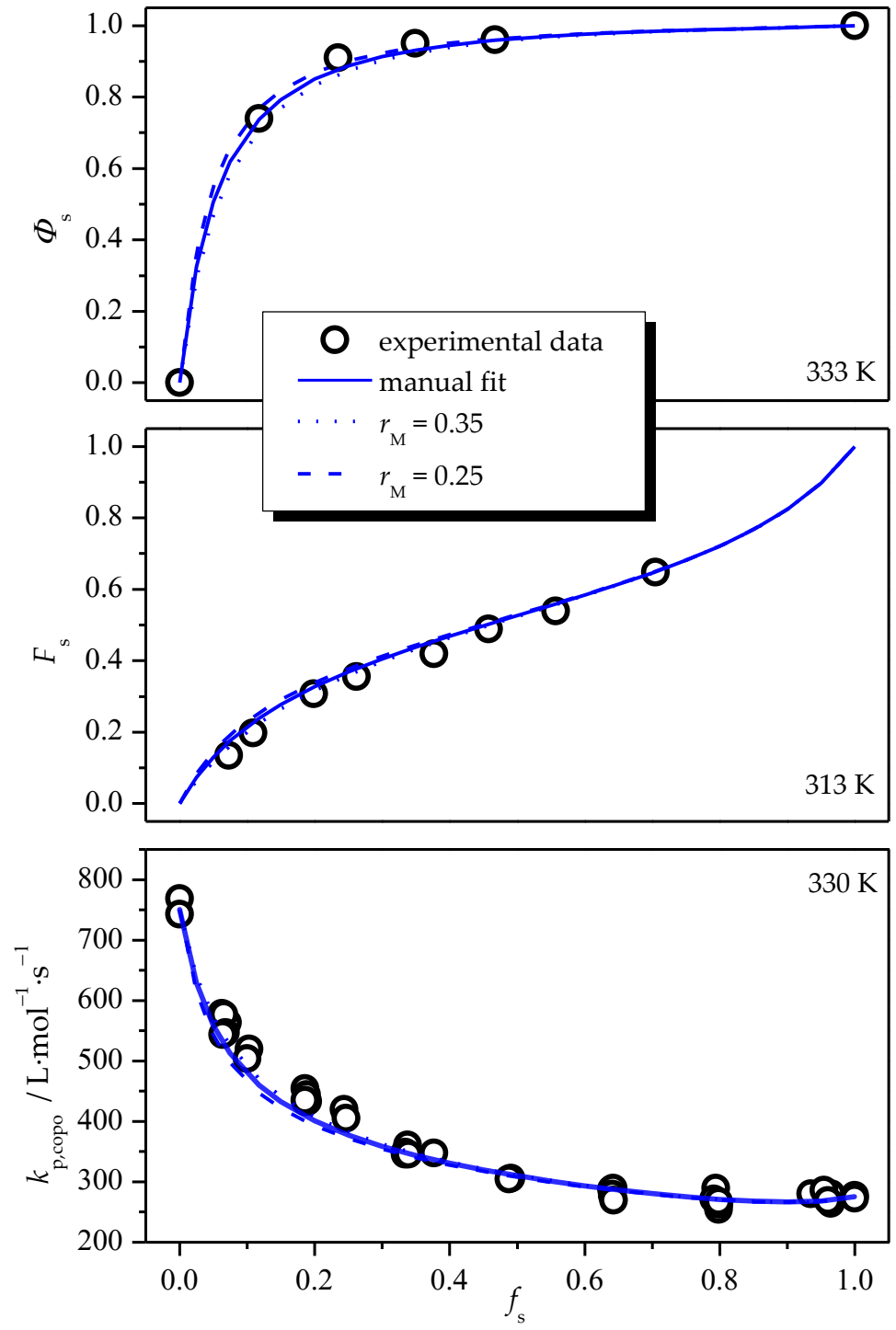

Figure II.10: Error estimation for the manually fitted copolymerization parameter $r_{\mathrm{m}}$ by the explicit penultimate model is shown as dotted and dashed blue lines, respectively. The manual fit with the copolymerization parameters given in Table 5.5 is shown as solid blue line. $F_{\mathrm{S}}{ }^{[50]}$ and the overall $k_{\mathrm{p}, \text { copo }}{ }^{[131]}$ were taken from literature. 

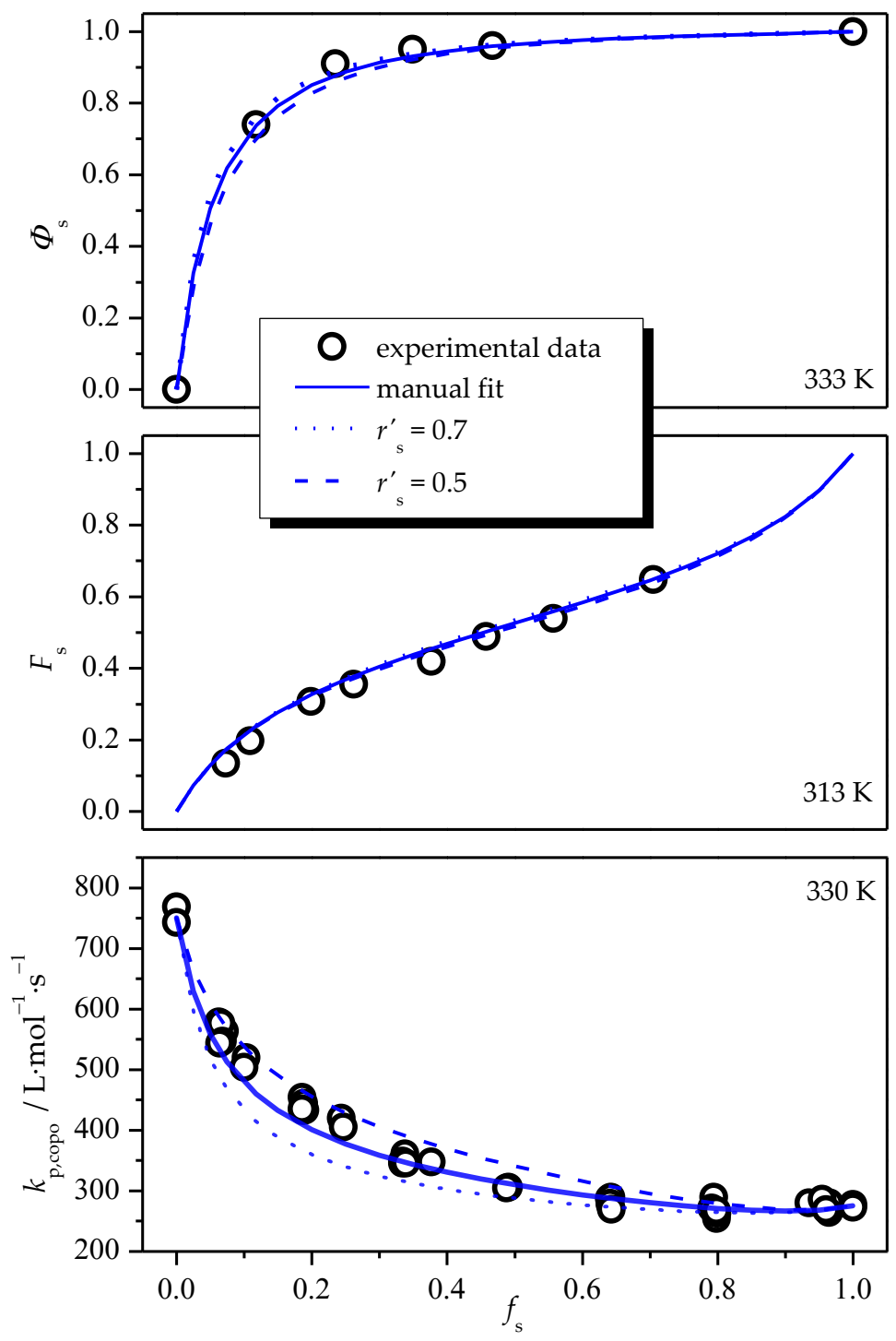

Figure II.11: Error estimation for the manually fitted copolymerization parameter $r_{\mathrm{S}}^{\prime}$ by the explicit penultimate model is shown as dotted and dashed blue lines, respectively. The manual fit with the copolymerization parameters given in Table 5.5 is shown as solid blue line. $F_{\mathrm{S}}^{[50]}$ and the overall $k_{\mathrm{p}, \text { copo }}{ }^{[131]}$ were taken from literature. 

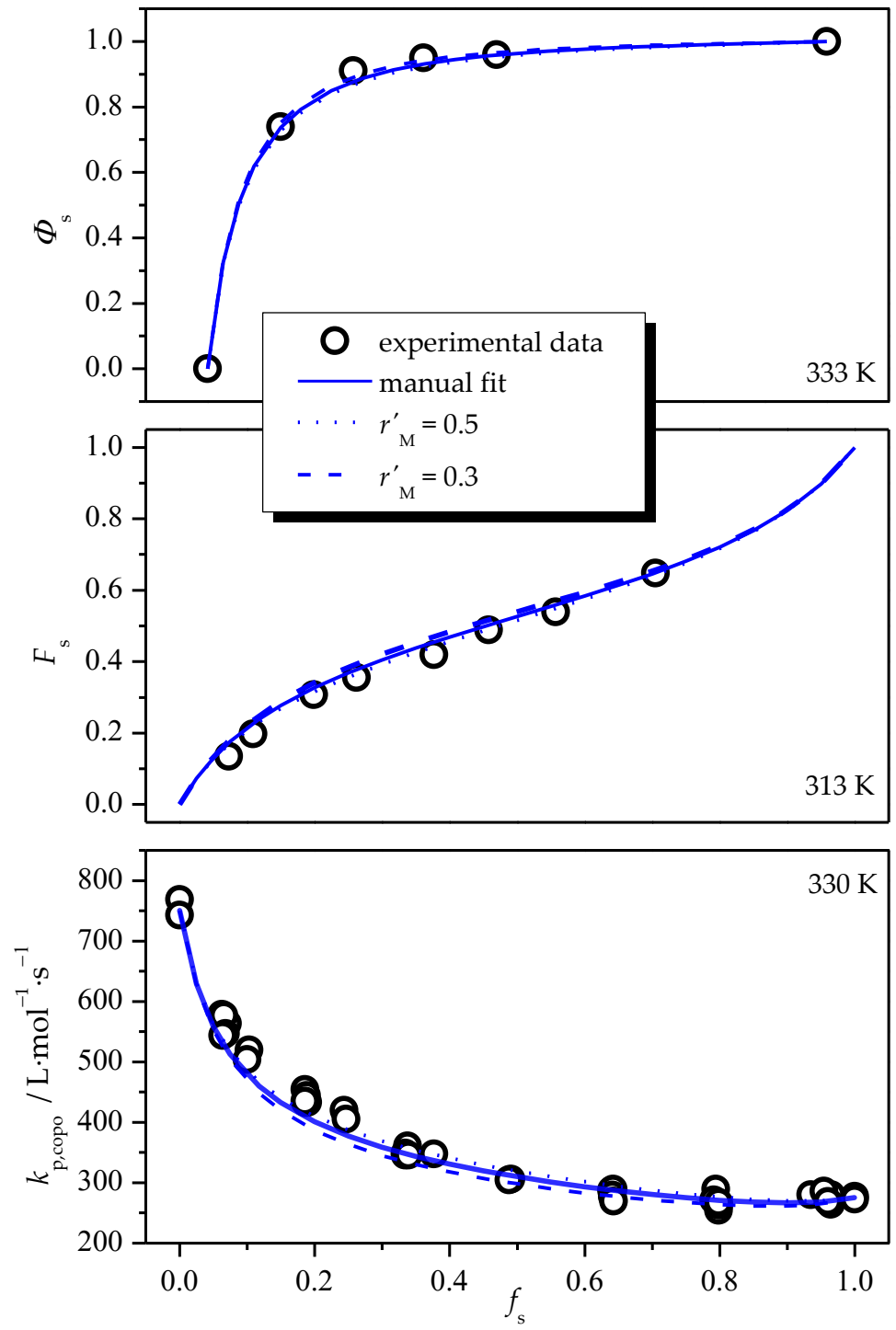

Figure II.12: Error estimation for the manually fitted copolymerization parameter $r_{\mathrm{M}}^{\prime}$ by the explicit penultimate model is shown as dotted and dashed blue lines, respectively. The manual fit with the copolymerization parameters given in Table 5.5 is shown as solid blue line. $F_{\mathrm{S}}{ }^{[50]}$ and the overall $k_{\mathrm{p}, \text { copo }}{ }^{[131]}$ were taken from literature. 


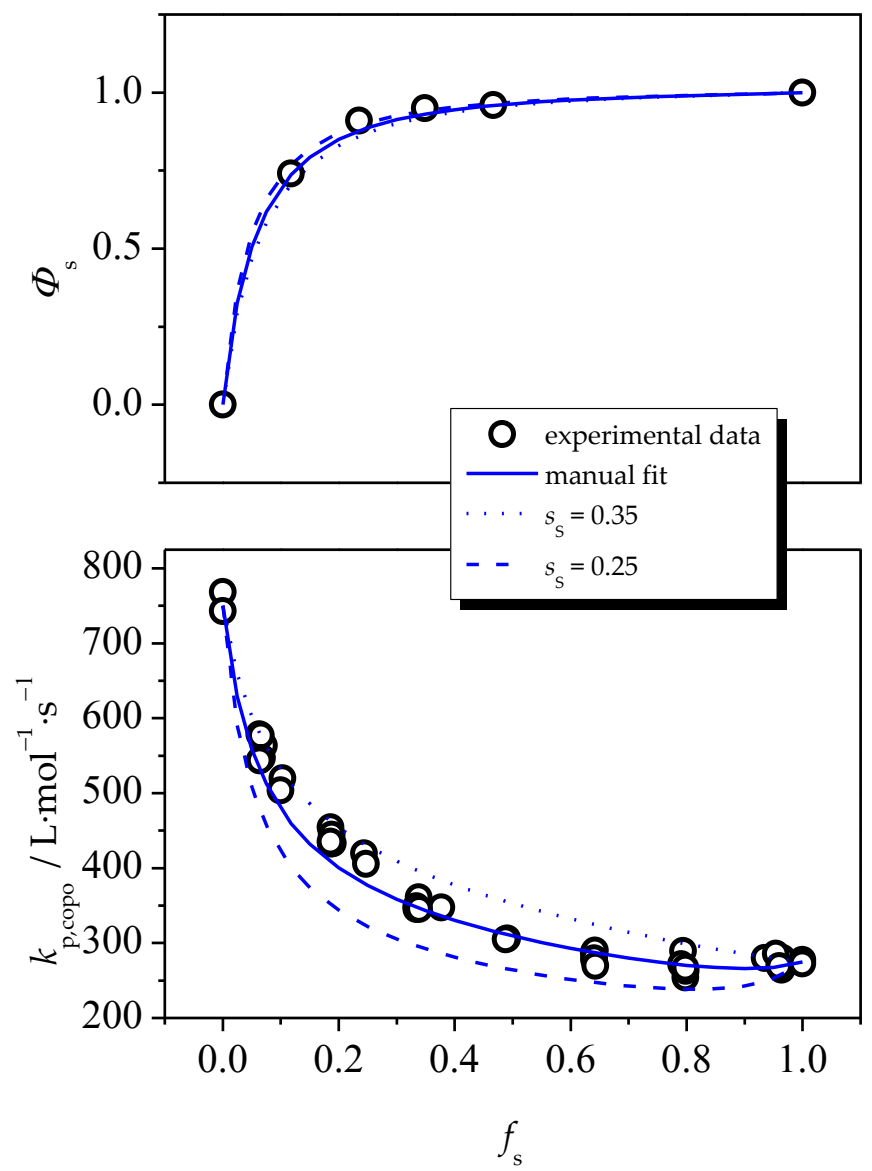

Figure II.13: Error estimation for the manually fitted copolymerization parameter $s_{\mathrm{S}}$ by the explicit penultimate model is shown as dotted and dashed blue lines, respectively. The manual fit with the copolymerization parameters given in Table 5.5 is shown as solid blue line. The overall $k_{\mathrm{p}, \text { copo }}{ }^{[131]}$ was taken from literature. 


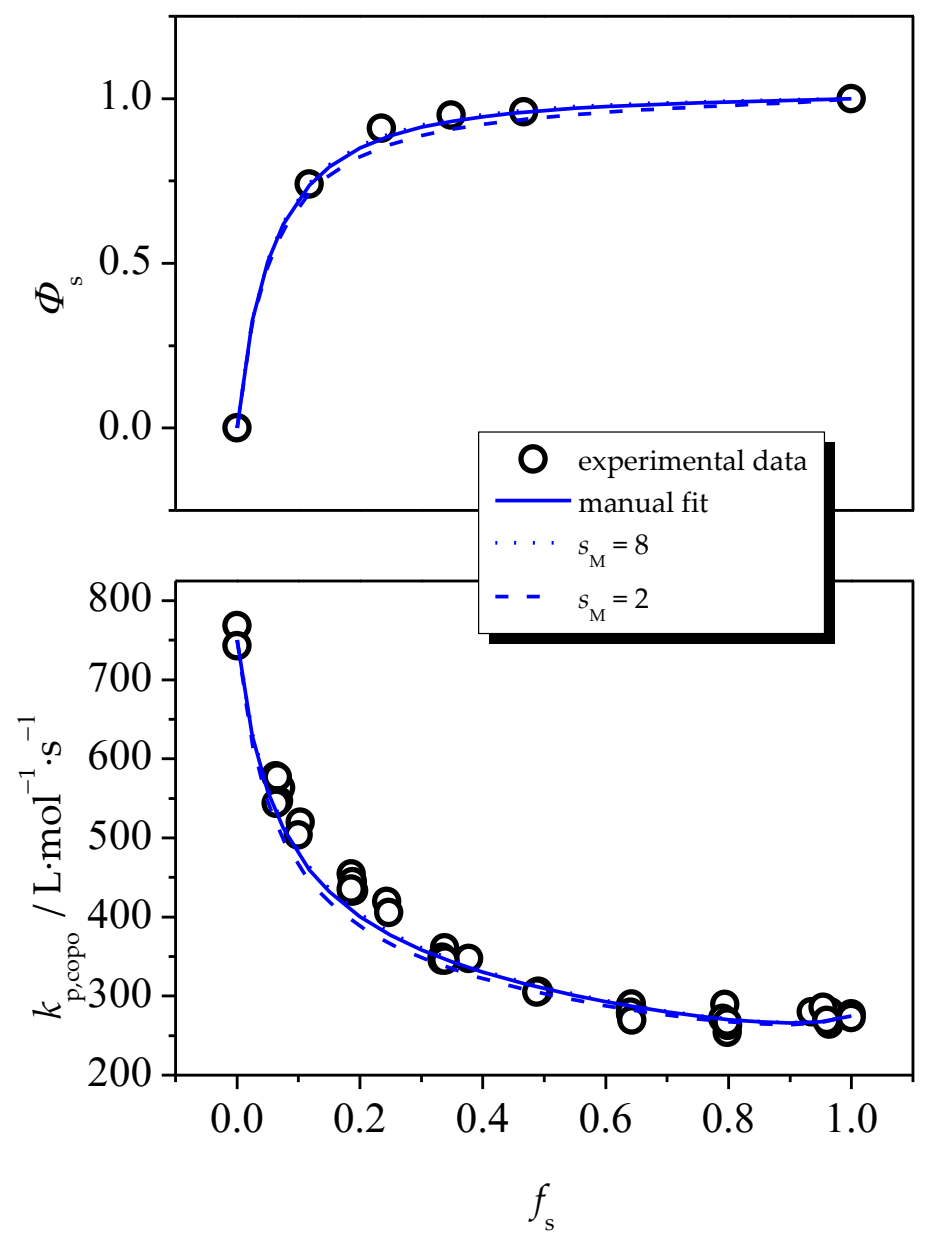

Figure II.14: Error estimation for the manually fitted copolymerization parameter $s_{\mathrm{M}}$ by the explicit penultimate model is shown as dotted and dashed blue lines, respectively. The manual fit with the copolymerization parameters given in Table 5.5 is shown as solid blue line. The overall $k_{\mathrm{p}, \text { copo }}{ }^{[131]}$ was taken from literature. 


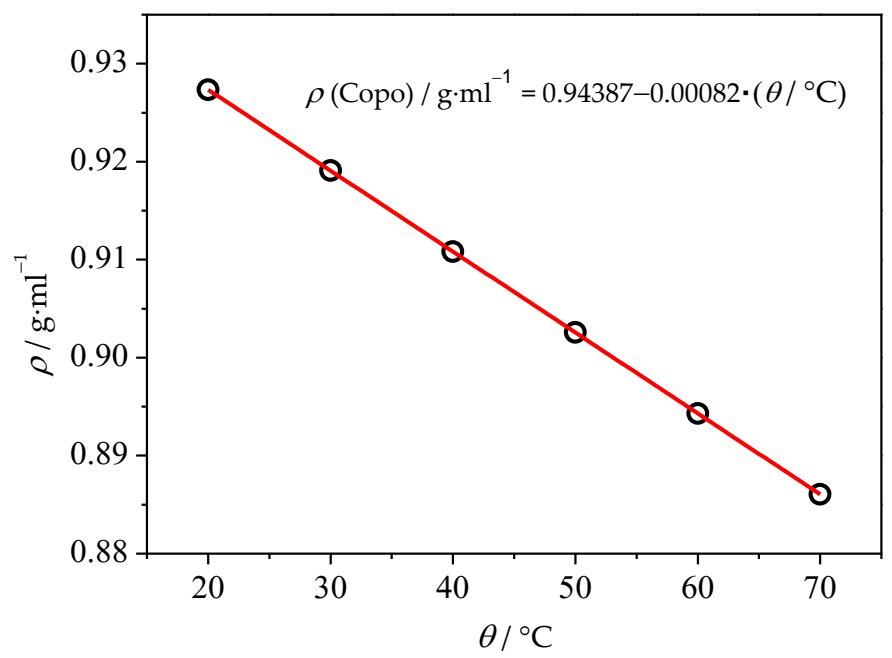

Figure II.15: Temperature dependence of the density for the copolymerization of styrene and MMA $\left(f_{\text {sty }}=0.467\right.$ and $\left.f_{\mathrm{MMA}}=0.533\right)$.

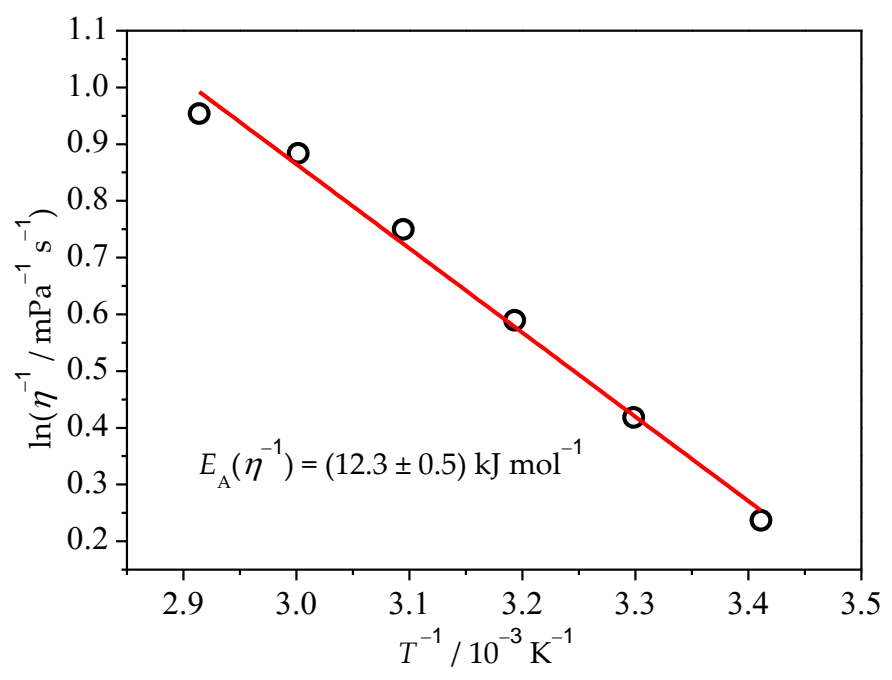

Figure II.16: Arrhenius plot of the fluidity and the determined activation energy $E_{\mathrm{A}}$ for the copolymerization of styrene and MMA $\left(f_{\text {sty }}=0.498\right.$ and $\left.f_{\mathrm{MMA}}=0.502\right)$ in bulk. 


\section{Matlab Simulations}

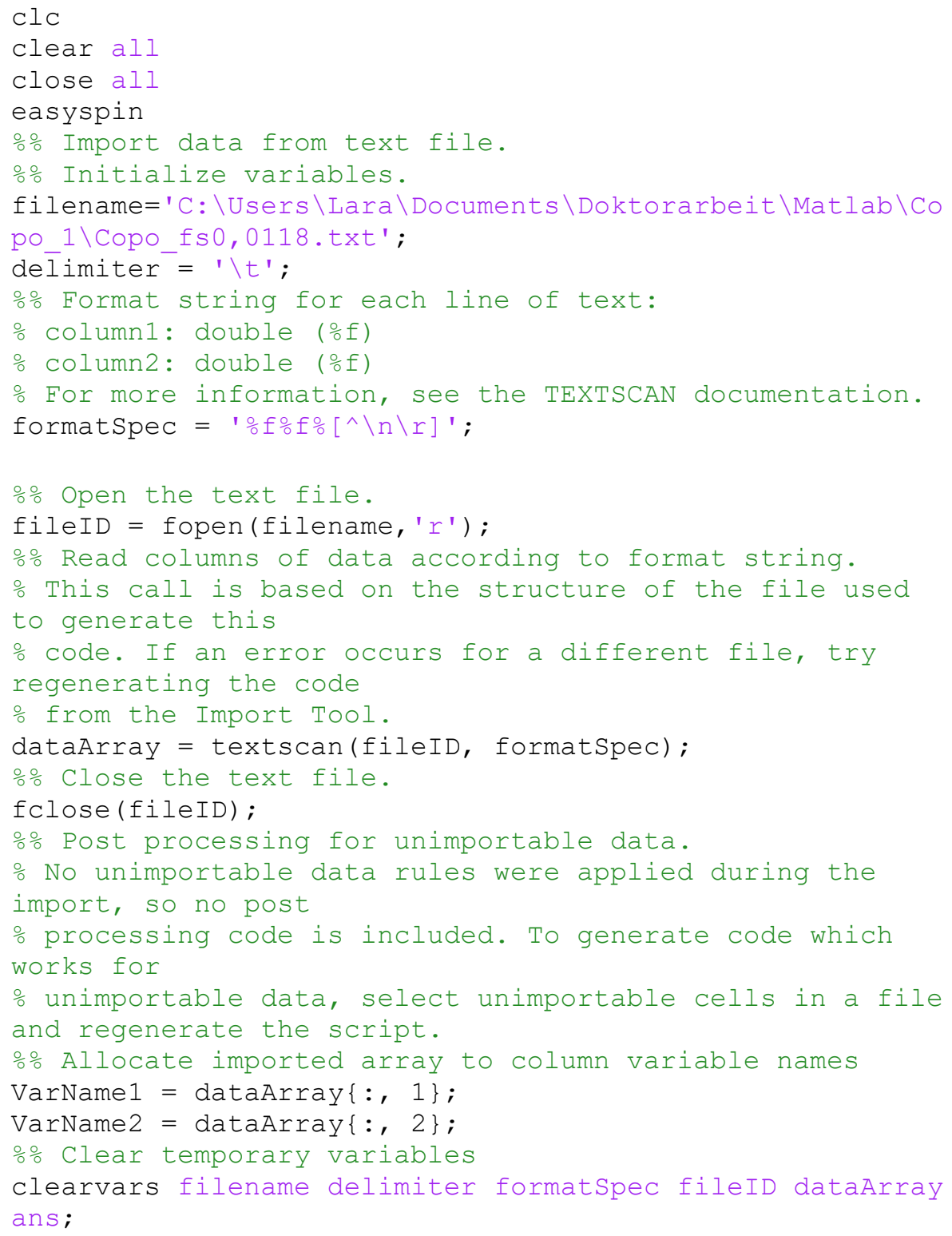




\section{Matlab Simulations}
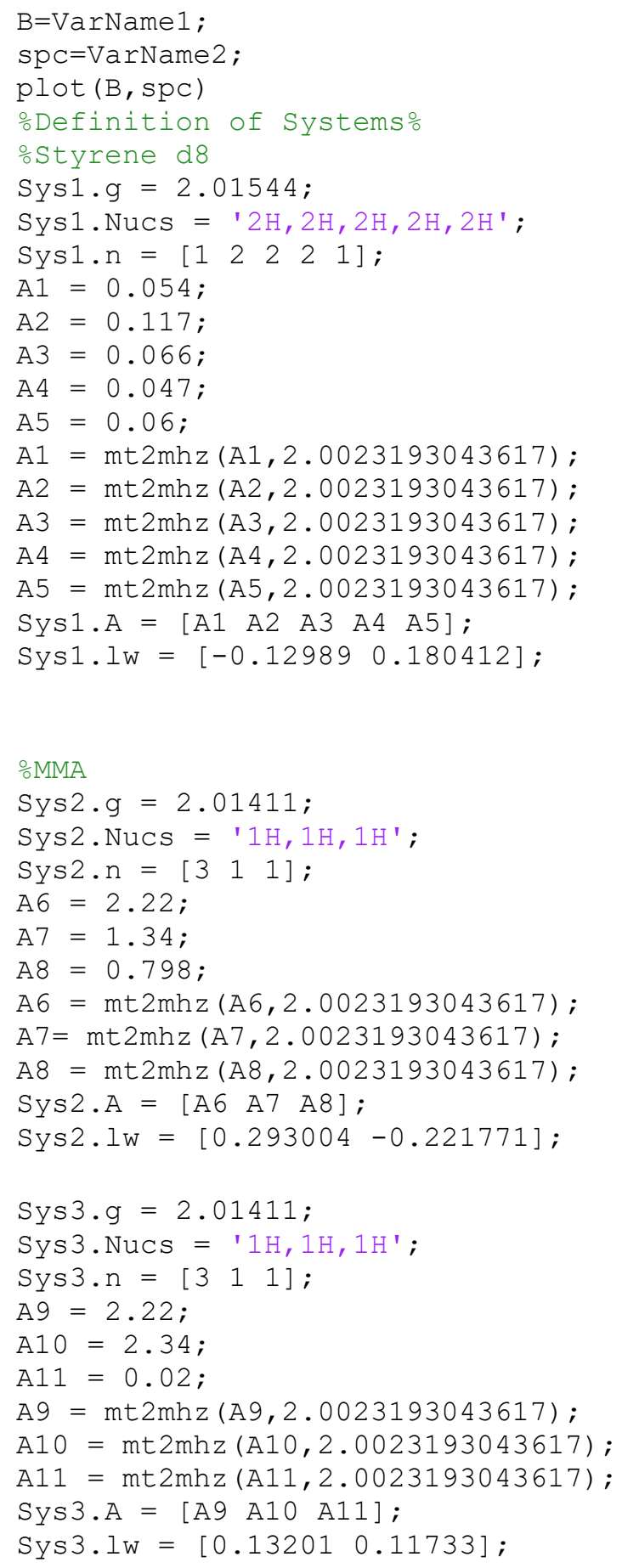


\section{Matlab Simulations}

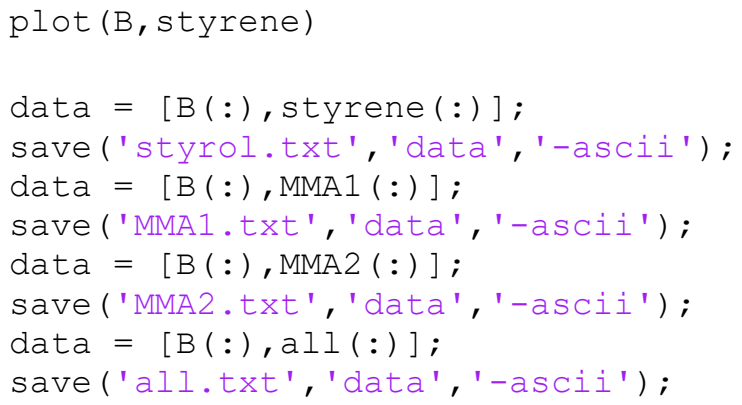





\section{LITERATURE}

[1] L. H. Baekeland, Chemiker-Zeitung 1909, 33, 317-318.

[2] H. Staudinger, Berichte d. D. Chem. Gesellschaft 1920, 53, 1073-1085.

[3] H. G. Elias, Makromoleküle Band 4: Anwendung von Polymeren, Wiley-VCH, Weinheim, 1999.

[4] G. Moad, D. H. Solomon, The Chemistry of Radical Polymerization, Elsevier, The Netherlands, 2006.

[5] K. Matyjaszewski, T. P. Davis, Handbook of Radical Polymerization, John Wiley And Sons, Inc., Hoboken, 2002.

[6] C. Barner-Kowollik, M. Buback, M. Egorov, T. Fukuda, A. Goto, O. F. Olaj, G. T. Russell, P. Vana, B. Yamada, P. B. Zetterlund, Prog. Polym. Sci. 2005, 30, 605-643.

[7] O. F. Olaj, I. Bitai, F. Hinkelmann, Macromol. Chem. 1987, 188, 16891702.

[8] M. Buback, R. G. Gilbert, R. A. Hutchinson, B. Klumperman, F.-D. Kuchta, B. G. Manders, K. F. O'Driscoll, G. T. Russell, J. Schweer, Macromol. Chem. Phys. 1995, 196, 3267-3280.

[9] S. Beuermann, M. Buback, T. P. Davis, R. G. Gilbert, R. A. Hutchinson, O. F. Olaj, G. T. Russell, J. Schweer, A. M. van Herk, Macromol. Chem. Phys. 1997, 198, 1545-1560.

[10] S. Beuermann, M. Buback, T. P. Davis, R. G. Gilbert, R. A. Hutchinson, A. Kajiwara, B. Klumperman, G. T. Russell, Macromol. Chem. Phys. 2000, 201, 1355-1364.

[11] T. P. Davis, K. F. O'Driscoll, M. C. Piton, M. A. Winnik, J. Polym. Sci. Part C Polym. Lett. 1989, 27, 181-185.

[12] T. P. Davis, K. F. O'Driscoll, M. C. Piton, M. A. Winnik, Macromolecules 1990, 23, 2113-2119. 
[13] R. A. Cockburn, T. F. L. McKenna, R. A. Hutchinson, Macromol. Chem. Phys. 2010, 211, 501-509.

[14] M. Dossi, K. Liang, R. A. Hutchinson, D. Moscatelli, J. Phys. Chem. B 2010, 114, 4213-4222.

[15] K. Liang, R. A. Hutchinson, Macromolecules 2010, 43, 6311-6320.

[16] T. P. Davis, K. F. O’Driscoll, M. C. Piton, M. A. Winnik, Polym. Int. 1991, 24, 65-70.

[17] R. A. Hutchinson, J. H. McMinn, D. A. Paquet, S. Beuermann, C. Jackson, Ind. Eng. Chem. Res. 1997, 36, 1103-1113.

[18] M. L. Coote, M. D. Zammit, T. P. Davis, G. D. Willett, Macromolecules 1997, 30, 8182-8190.

[19] M. L. Coote, T. P. Davis, Macromolecules 1999, 32, 3626-3635.

[20] M. Buback, A. Feldermann, C. Barner-Kowollik, I. Lacík, Macromolecules 2001, 34, 5439-5448.

[21] L. H. Yee, J. P. A. Heuts, T. P. Davis, Macromolecules 2001, 34, 35813586.

[22] L. M. Morris, T. P. Davis, R. P. Chaplin, Polymer (Guildf). 2001, 42, 941-952.

[23] M. Buback, E. Müller, Macromol. Chem. Phys. 2007, 208, 581-593.

[24] S. Beuermann, M. Buback, Prog. Polym. Sci. 2002, 27, 191-254.

[25] M. Buback, H. Schroeder, H. Kattner, Macromolecules 2016, 49, 3193-3213.

[26] M. Buback, M. Egorov, T. Junkers, E. Panchenko, Macromol. Rapid Commun. 2004, 25, 1004-1009.

[27] H. Kattner, M. Buback, Macromol. Symp. 2013, 333, 11-23.

[28] J. Barth, M. Buback, Macromol. React. Eng. 2010, 4, 288-301.

[29] G. B. Smith, G. T. Russell, J. P. A. Heuts, Macromol. Theory Simulations 2003, 12, 299-314.

[30] J. Barth, M. Buback, Macromol. Rapid Commun. 2009, 30, 1805-1811. 
[31] J. Barth, M. Buback, P. Hesse, T. Sergeeva, Macromolecules 2009, 42, 481-488.

[32] M. Buback, E. Müller, G. T. Russell, J. Phys. Chem. A 2006, 110, 3222-3230.

[33] M. Buback, M. Egorov, T. Junkers, E. Panchenko, Macromol. Chem. Phys. 2005, 206, 333-341.

[34] H. Kattner, M. Buback, Macromolecules 2015, 48, 309-315.

[35] H. Kattner, M. Buback, Macromolecules 2017, 50, 5308-5314.

[36] J. Barth, M. Buback, P. Hesse, T. Sergeeva, Macromol. Rapid Commun. 2009, 30, 1969-1974.

[37] J. Barth, M. Buback, Macromolecules 2012, 45, 4152-4157.

[38] P. Drawe, H. Kattner, M. Buback, Macromol. Chem. Phys. 2016, 2755-2764.

[39] H. Kattner, M. Buback, Macromol. Rapid Commun. 2015, 36, 21862191.

[40] H. Kattner, P. Drawe, M. Buback, Macromolecules 2017, 50, 1386-1393.

[41] J. Barth, M. Buback, P. Hesse, T. Sergeeva, Macromolecules 2010, 43, 4023-4031.

[42] J. Barth, M. Buback, G. T. Russell, S. Smolne, Macromol. Chem. Phys. 2011, 212, 1366-1378.

[43] H. Kattner, M. Buback, Macromolecules 2018, 51, $25-33$.

[44] J. Barth, M. Buback, W. Meiser, P. Vana, Macromolecules 2010, 43, $51-54$.

[45] W. Meiser, M. Buback, Macromol. Rapid Commun. 2011, 32, 14901494.

[46] W. Meiser, M. Buback, O. Ries, C. Ducho, A. Sidoruk, Macromol. Chem. Phys. 2013, 214, 924-933.

[47] H. Kattner, P. Drawe, M. Buback, Macromol. Chem. Phys. 2015, 216, 1737-1745. 
[48] M. D. Lechner, K. Gehrke, E. H. Nordmeier, Makromolekulare Chemie, Brinkhäuser Verlag, Basel Boston Berlin, 2010.

[49] D. Li, N. Li, R. A. Hutchinson, Macromolecules 2006, 39, 4366-4373.

[50] T. Fukuda, H. Inagaki, Macromolecules 1985, 18, 17-26.

[51] Y.-D. Ma, T. Fukuda, H. Inagaki, Macromolecules 1985, 18, 26-31.

[52] M. L. Coote, L. P. M. Johnston, T. P. Davis, Macromolecules 1997, 30, 8191-8204.

[53] O. F. Olaj, M. Zoder, P. Vana, G. Zifferer, Macromolecules 2004, 37, 1544-1550.

[54] A. Nitschke, Investigation of Polymer-Filler Interactions Using Functionalized Nanoparticles, PhD Thesis, Georg-August Universität Göttingen, 2020.

[55] N. Sörensen, Kinetics and Mechanism of Cu-Catalyzed Atom Transfer Radical Polymerization, $\mathrm{PhD}$ Thesis Georg-August-Universität Göttingen, 2015.

[56] H. Kattner, Radical Polymerization Kinetics of Non-Ionized and FullyIonized Monomers Studied by Pulsed-Laser EPR, PhD Thesis GeorgAugust Universität Göttingen, 2016.

[57] D. S. Achilias, Macromol. Theory Simulations 2007, 16, 319-347.

[58] O. F. Olaj, H. F. Kauffmann, J. W. Breitenbach, Makromol. Chem. 1977, 178, 2707-2717.

[59] M. Buback, R. G. Gilbert, G. T. Russell, D. J. T. Hill, G. Moad, K. F. O'Driscoll, J. Shen, M. A. Winnik, J. Polym. Sci. Part A Polym. Chem. 1992, 30, 851-863.

[60] R. Szymanski, Macromol. Theory Simulations 2011, 20, 8-12.

[61] T. Junkers, C. Barner-Kowollik, J. Polym. Sci. Part A Polym. Chem. 2008, 46, 7585-7605.

[62] M. Buback, Die Makromol. Chemie 1990, 191, 1575-1587.

[63] S. W. Benson, A. M. North, J. Am. Chem. Soc. 1959, 81, 1339-1345.

[64] S. W. Benson, A. M. North, J. Am. Chem. Soc. 1962, 84, 935-940. 
[65] R. X. E. Willemse, B. B. P. Staal, A. M. van Herk, S. C. J. Pierik, B. Klumperman, Macromolecules 2003, 36, 9797-9803.

[66] O. F. Olaj, P. Vana, M. Zoder, A. Kornherr, G. Zifferer, Macromol. Rapid Commun. 2000, 21, 913-920.

[67] O. F. Olaj, P. Vana, M. Zoder, Macromolecules 2002, 35, 1208-1214.

[68] G. B. Smith, G. T. Russell, M. Yin, J. P. A. Heuts, Eur. Polym. J. 2005, $41,225-230$.

[69] J. P. A. Heuts, R. G. Gilbert, L. Radom, Macromolecules 1995, 28, 8771-8781.

[70] C. Barner-Kowollik, G. T. Russell, Prog. Polym. Sci. 2009, 34, 12111259.

[71] M. v. Smoluchowski, Zeitschrift für Phys. Chemie 1918, 92, 129-168.

[72] H. Paul, H. Fischer, Acc. Chem. Res. 1987, 20, 200-206.

[73] H. G. Elias, Makromoleküle Band 1: Chemische Struktur Und Synthese, Wiley-VCH, Weinheim, 1999.

[74] H. Fischer, L. Radom, Angew. Chemie 2001, 113, 1380-1414.

[75] F. R. Mayo, F. M. Lewis, J. Am. Chem. Soc. 1944, 66, 1594-1601.

[76] T. Alfrey, G. Goldfinger, J. Chem. Phys. 1944, 12, 205-209.

[77] D. J. T. Hill, J. H. O'Donnell, P. W. O'Sullivan, Macromolecules 1982, 15, 960-966.

[78] E. Merz, T. Alfrey, G. Goldfinger, J. Polym. Sci. 1946, 1, 75-82.

[79] T. Fukuda, Y. D. Ma, H. Inagaki, K. Kubo, Macromolecules 1991, 24, 370-375.

[80] T. Fukuda, K. Kubo, Y.-D. Ma, Prog. Polym. Sci. 1992, 17, 875-916.

[81] M. L. Coote, T. P. Davis, L. Radom, J. Mol. Struct. 1999, 461-462, 91-96.

[82] M. L. Coote, T. P. Davis, L. Radom, Macromolecules 1999, 32, 29352940. 
[83] M. L. Coote, T. P. Davis, L. Radom, Macromolecules 1999, 32, 52705276.

[84] H. Tanaka, K. Sasai, T. Sato, T. Ota, Macromolecules 1988, 21, 35343536.

[85] T. Sato, K. Takahashi, H. Tanaka, T. Ota, K. Kato, Macromolecules 1991, 24, 2330-2333.

[86] T. P. Davis, J. Polym. Sci. Part A Polym. Chem. 2001, 39, 597-603.

[87] C. Walling, J. Am. Chem. Soc. 1949, 71, 1930-1935.

[88] J. N. Atherton, A. M. North, Trans. Faraday Soc. 1962, 58, 2049-2057.

[89] K. Ito, K. F. O’Driscoll, J. Polym. Sci. Polym. Chem. Ed. 1979, 17, 3913-3921.

[90] Y. D. Ma, K. S. Sung, Y. Tsujii, T. Fukuda, Macromolecules 2001, 34, 4749-4756.

[91] G. B. Smith, G. T. Russell, Zeitschrift für Phys. Chemie 2005, 219, 295323.

[92] P. Vana, T. P. Davis, C. Barner-Kowollik, Aust. J. Chem. 2002, 55, 315-318.

[93] M. Buback, A. Kuelpmann, Macromol. Chem. Phys. 2003, 204, 632637.

[94] H. Kattner, M. Buback, Macromol. Chem. Phys. 2014, 215, 1180-1191.

[95] E. Rizzardo, A. K. Serelis, D. H. Solomon, Aust. J. Chem. 1982, 35, 2013-2024.

[96] H. Kattner, Untersuchung der radikalischen Polymerisation von Vinylacetat durch Pulslaserexperimente in Verbindung mit EPR und SEC, Master Thesis, Georg-August Universität Göttingen, 2012.

[97] P. D. Zeuch, Thesis Comitee Meeting, 2017.

[98] A. P. Haehnel, M. Schneider-Baumann, L. Arens, A. M. Misske, F. Fleischhaker, C. Barner-Kowollik, Macromolecules 2014, 47, 34833496. 
[99] A. Nitschke, L. Riemann, L. Kollenbach, V. Braun, M. Buback, P. Vana, Macromol. Chem. Phys. 2020, 221, 1900345.

[100] A. Groschopp, Kinetik der radikalischen Polymerisation von Monomeren mit mesogener Seitengruppe in isotroper und anisotroper Lösung, PhD Thesis, Georg-August Universität Görringen 2018.

[101] C. Heller, H. M. McConnell, J. Chem. Phys. 1960, 32, 1535-1539.

[102] S. Dai, J. Chem. Educ. 1991, 68, 894-895.

[103] L. Hermosilla, P. Calle, C. Sieiro, N. García, P. Tiemblo, J. Guzmán, Chem. Phys. 2007, 340, 237-244.

[104] M. Buback, T. Junkers, Macromol. Chem. Phys. 2006, 207, 1640-1650.

[105] O. F. Olaj, P. Vana, Macromol. Rapid Commun. 1998, 19, 533-538.

[106] J. G. Kirkwood, J. Riseman, J. Chem. Phys. 1948, 16, 565-573.

[107] G. Johnston-Hall, M. J. Monteiro, J. Polym. Sci. Part A Polym. Chem. 2008, 46, 3155-3173.

[108] B. Friedman, B. O'Shaughnessy, Macromolecules 1993, 26, 57265739.

[109] R. A. Hutchinson, S. Beuermann, D. A. Paquet, J. H. McMinn, Macromolecules 1997, 30, 3490-3493.

[110] G. Johnston-Hall, A. Theis, M. J. Monteiro, T. P. Davis, M. H. Stenzel, C. Barner-Kowollik, Macromol. Chem. Phys. 2005, 206, 2047-2053.

[111] S. Smolne, S. Weber, M. Buback, Macromol. Chem. Phys. 2016, 217, 2391-2401.

[112] J. P. A. Heuts, D. Kukulj, D. J. Forster, T. P. Davis, Macromolecules 1998, 31, 2894-2905.

[113] L. Riemann, Untersuchung der Terminierungskinetik von Radikalischen Copolymerisationen mittels zeitaufgelöster ESRSpektroskopie, Master Thesis, Georg-August Universität Göttingen, 2016. 
[114] P. B. Zetterlund, S. Tagashira, K. Izumi, Y. Nagano, M. Azukizawa, H. Yamazoe, M. Kumagai, B. Yamada, Macromolecules 2002, 35, 8209-8215.

[115] V. B. Golubev, V. P. Zubov, G. S. Georgiev, I. L. Stoyachenko, V. A. Kabanov, J Polym Sci Part A-1 Polym Chem 1973, 11, 2463-2487.

[116] A. Kajiwara, S. Arata, K. Maeda, H. Nakajima, S. Yamabe, Polym. Prepr. 2008, 49, 32-33.

[117] B. Yamada, M. Kageoka, T. Otsu, Macromolecules 1992, 25, 48284831.

[118] B. Yamada, S. Tagashira, K. Sakamoto, Y. Nagano, Y. Miura, Polym. Bull. 1997, 38, 339-346.

[119] S. A. Fairhurst, R. S. Pilkington, L. H. Sutcliffe, J. Chem. Soc. Faraday Trans. 1 1983, 79, 925-940.

[120] G. Clouet, P. Chaffanjon, J. Macromol. Sci. Part A - Chem. 1990, 27, 193-212.

[121] M. L. Coote, T. P. Davis, B. Klumperman, M. J. Monteiro, J. Macromol. Sci. Part C Polym. Rev. 1998, 38, 567-593.

[122] J. A. Seiner, M. Litt, Macromolecules 1971, 4, 308-311.

[123] R. E. Cais, R. G. Farmer, D. J. T. Hill, J. H. O'Donnell, Macromolecules 1979, 12, 835-839.

[124] P. G. Brown, K. Fujimori, J. Polym. Sci. Part A Polym. Chem. 1994, 32, 2971-2978.

[125] T. Fukuda, K. Kubo, Y.-D. Ma, H. Inagaki, Polym. J. 1987, 19, 523530.

[126] B. Klumperman, K. F. O'Driscoll, Polymer (Guildf). 1993, 34, 10321037.

[127] B. Klumperman, I. R. Kraeger, Macromolecules 1994, 27, 1529-1534.

[128] I. A. Maxwell, A. M. Aerdts, A. L. German, Macromolecules 1993, 26, 1956-1964.

[129] H. Kattner, M. Buback, Macromolecules 2017, 50, 4160-4168. 
[130] H. A. S. Schoonbrood, S. C. J. Pierik, B. Van Den Reijen, J. P. A. Heuts, A. L. German, Macromolecules 1996, 29, 6717-6723.

[131] M. L. Coote, The Origin of the Penultimate Unit Effect in Free-Radical Copolymerization, PhD Thesis, University of New South Wales Sydney, Australia, 1999.

[132] J. Schweer, Die Makromol. Chemie, Theory Simulations 1993, 2, 485502.

[133] M. S. Kent, M. Tirrell, T. P. Lodge, J. Polym. Sci. Part B Polym. Phys. 1994, 32, 1927-1941. 



\section{Acknowledgements}

Als erstes möchte ich Herrn Prof. Dr. Philipp Vana für die Betreuung in den letzten Jahren bedanken. Ebenso möchte ich mich für das mir entgegengebrachte Vertrauen und die Unterstützung bedanken, besonders bei den zahlreichen apparativen Problemen. Des Weiteren möchte ich mich bei Herrn Prof. Dr. Thomas Zeuch für die Übernahme des Korreferates und den hilfreichen Ratschlägen bedanken.

Herrn Prof. Dr. Michael Buback, Herrn Prof. Dr. Burkhard Geil, Herrn Prof. Dr. Dietmar Stalke und Dr. Tim Schäfer danke ich, dass sie Teil meiner Prüfungskommission sind. Ein besonderer Dank gilt an dieser Stelle Herm Prof. Dr. Michael Buback für die vielen und hilfreichen Diskussionen und Ratschläge.

Bei allen aktuellen und ehemaligen Mitgliedern des Arbeitskreises Vana möchte ich mich für die großartige Arbeitsatmosphäre bedanken. Die gemeinsamen kulinarischen Kocherlebnisse und Spielerunden waren eine willkommene Abwechslung zum Laboralltag. Insbesondere möchte ich mich bei Dr. Annika Nitschke für die tolle Zusammenarbeit im Rahmen unserer gemeinsamen Publikation bedanken. Ein großer Dank gilt auch meinen beiden Bachelorstudenten Sarah Brandt und Theodor Galambos, die beide einen großen Beitrag $\mathrm{zu}$ dieser Arbeit geleistet haben. Meinen fleißigen Korrekturlesern Dr. Judith Rauschendorfer, Stella Weber, Enno Meyer, Darius Rohleder und Dr. Peng Wentao danke ich auch herzlich für die Unterstützung. Für den Support rundum die Apparaturen und Praktika möchte ich mich bei Heike Rohmann, Sandra Lotze, Dr. Hans-Peter Vögele und Dr. Florian Ehlers bedanken.

Des Weiteren möchte ich mich bei Eva Dušička vom Polymer Institute in Bratislava für die Kooperation bedanken. Auch wenn durch Corona und Probleme mit der Apparatur die Arbeit nicht komplementiert werden konnte, war die Zusammenarbeit sehr gut. Meinem „Telefonjoker“ Dr. Hendrik Kattner danke ich für die Unterstützung aus der Ferne.

Ohne meine Familie und Freunde wäre ich nicht so weit gekommen. Besonders möchte ich meinen Eltern für die bedingungslose Unterstützung in allen Lebenslagen danken. Meine Großeltern und Brüder haben auch immer für 
einen enormen Rückhalt gesorgt, wofür ich sehr dankbar bin. Ich bin auch dankbar, dass ich so tolle Freunde habe, die mir immer Mut zugesprochen haben und mich aufgemuntert haben, wenn es mal wieder nicht so lief. Abschließend möchte ich Stephan für die vielen Urlaube, Aktivitäten und Renovierungsarbeiten, die vom Unialltag abgelenkt haben, bedanken. Ich freue mich auf unsere gemeinsame Zukunft! 


\section{About the Author}

Lara Riemann, *April 9, 1991, Witzenhausen, German citizen.

Curriculum vitae

2010

Abitur, Berufliche Schulen des Werra Meißner Kreises, Witzenhausen, focus on Biotechnology

2010-2013 Bachelor of Science, Georg-August-University Göttingen, Bachelor Thesis: "Signalverbreiterung in der Gelpermeationschromatographie: Einfluss auf die Molmassenbestimmung von Polymeren"

2013-2016 Master of Science, Georg-August-University Göttingen, Master Thesis: „Untersuchung der Terminierungskinetik von radikalischen Copolymerisationen mittels zeitaufgelöster ESR-Spektroskopie“"

2016-2021 Doctoral Studies, Georg-August-University Göttingen, supervisor: Prof. Dr. Philipp Vana, MBA

Publications

"Investigation into the Kinetics of $n$-Pentyl Methacrylate Radical Polymerization"

A. Nitschke, L. Riemann, L. Kollenbach, V. Braun, M. Buback, P. Vana, Macromol. Chem. Phys. 2020, 221, 1900345.

\section{Contributions to Conferences}

Poster, Bordeaux Polymer Conference, Bordeaux, France, May 2018.

Poster, JungChemiker Forum, Göttingen July 2019. 